\title{
Design and analysis of a hybrid guideway heating system for Morgantown Personal Rapid Transit
}

Anand Vedam

West Virginia University

Follow this and additional works at: https://researchrepository.wvu.edu/etd

\section{Recommended Citation}

Vedam, Anand, "Design and analysis of a hybrid guideway heating system for Morgantown Personal Rapid Transit" (2009). Graduate Theses, Dissertations, and Problem Reports. 2026.

https://researchrepository.wvu.edu/etd/2026

This Thesis is protected by copyright and/or related rights. It has been brought to you by the The Research Repository @ WVU with permission from the rights-holder(s). You are free to use this Thesis in any way that is permitted by the copyright and related rights legislation that applies to your use. For other uses you must obtain permission from the rights-holder(s) directly, unless additional rights are indicated by a Creative Commons license in the record and/ or on the work itself. This Thesis has been accepted for inclusion in WVU Graduate Theses, Dissertations, and Problem Reports collection by an authorized administrator of The Research Repository @ WVU. For more information, please contact researchrepository@mail.wvu.edu. 


\title{
Design and Analysis of a Hybrid Guideway Heating System for
} Morgantown Personal Rapid Transit

\author{
Anand Vedam \\ Thesis submitted to the \\ College of Engineering and Mineral Resources \\ at West Virginia University \\ in partial fulfillment of the requirements \\ for the degree of \\ Master of Science \\ in \\ Mechanical Engineering \\ John E. Sneckenberger, Ph.D., Chair \\ Bruce Kang, Ph.D. \\ Ali Feliachi, Ph.D
}

Department of Mechanical and Aerospace Engineering

Morgantown, West Virginia

2009

Keywords: Hybrid Heating System, Fuel Cell, Natural Gas, Guideway and Theoretical Analysis 


\title{
ABSTRACT \\ Design and Analysis of a Hybrid Guideway Heating System for Morgantown Personal Rapid Transit
}

\begin{abstract}
Anand Vedam
The Morgantown Personal Rapid Transit (M-PRT) system is a comfortable conveyance for travel in Morgantown, WV. One of its operating concerns is the increasing cost of heat to the guideway during winter. As the vehicles cannot run safely during snow, the system includes a guideway heating system to melt the ice from the guideway. To reduce the use of expensive natural gas, an interest has been expressed to define a hybrid heating system using an alternate fuel supply. Solid Oxide Fuel Cell (SOFC) was incorporated in the hybrid heating system. This hybrid heating system was designed, and then a detailed analysis was performed to ascertain the performance parameters like heat produced, thermal efficiency, cost of the system and the emissions involved. This high temperature fuel cell releases large amounts of usable heat in the form of exhaust gases. The exhaust gases are deprived of any undesired emissions that pollute the atmosphere. A USDOE EPSCoR WV State Implementation Award conducted by Advance Power Electricity Research Center (APERC) at West Virginia University provided support for conducting this research.
\end{abstract}




\section{ACKNOWLEDGEMENTS}

First and foremost, I thank God for providing me with an opportunity to pursue higher education. I thank my parents, my brother and sister for extending their support and encouragement. My special thanks to my wife for her love and co-operation.

I would like to express my sincere gratitude and appreciation to Dr. John E. Sneckenberger, my advisor and committee chairman. His contributions are numerous and valuable. Without his guidance and contributions, this thesis wouldn't have been possible. His encouragement will never be forgotten. I would like to thank the other committee members, Dr. Ali Feliachi for leading the Advance Power Electricity Research Center (APERC) projects and Dr. Bruce Kang for his valuable comments. I also thank all the APERC members for sharing their views about my project during my presentations and discussions.

I appreciate and thank Mr. Paul Harman and Mr. Robert Hendershot from WVU MPRT for providing all the necessary information for M-PRT guideway. I thank Padha Srinivasan, from UTC Power, for providing information about PureCell in UTC Power.

A USDOE EPSCoR WV State Implementation Award conducted by APERC at West Virginia University provided support for conducting this research. 


\section{TABLE OF CONTENTS}

$\begin{array}{ll}\text { ABSTRACT } & \text { ii }\end{array}$

ACKNOWLEDGEMENTS

TABLE OF CONTENTS iv

LIST OF FIGURES viii

LIST OF TABLES $\quad \mathrm{x}$

Chapter 1. Introduction 1

1.1 Guideway Heating System for Morgantown Personal Rapid Transit 1

1.2 Fuel Cell as a Source of Heat 2

1.3 Technology Applications for M-PRT by FTA and WVU 3

1.4 Research Objective 3

Chapter 2. Solid Oxide Fuel Cell as a Source of Heat 5

2.1 Performance Comparison of Different Fuel Cells 5

2.2 High Temperature Fuel Cells 5

2.3 Advantages of SOFC 7

2.4 Disadvantages of SOFC 7

2.5 Reason for Choosing SOFC 8

Chapter 3. Project Statement and Approach 9

3.1 Phase 1 of FTA Project 9

3.2 Goal for Project 10

3.3 Natural Gas in Existing Guideway Heating System 10

3.4 Difficulty with Existing Guideway Heating System 11

3.5 Approach for Hybrid Heating System 13

$\begin{array}{lll}3.6 & \text { List of Assumptions } & 13\end{array}$ 
Chapter 4. Thermodynamic Analysis of Existing Guideway Heating System 15

4.1 Purpose of Guideway Heating System 15

4.2 Guideway Heating System in the Morgantown Personal Rapid Transit 15

4.3 Thermodynamic Analysis of Existing Heating System 16

$\begin{array}{lll}\text { 4.3.1 Hours and Rate of Snowfall } & 18\end{array}$

$\begin{array}{ll}\text { 4.3.2 Heat Required by Guideway } & 18\end{array}$

4.3.3 Derivation of Heat Output from Existing Heating System 20

4.4 Emissions from Existing Heating System 28

\section{Chapter 5. Design of Hybrid Guideway Heating System 29}

$\begin{array}{lll}5.1 & \text { Introduction } & 29\end{array}$

5.2 Characteristics of Hybrid Guideway Heating System 29

5.3 Incorporation of Fuel Cell as a Source of Heat to Existing Heating 30 System

5.4 Fuel Cell as a Source of Heat 33

5.4.1 Specifications of Fuel Cell 33

5.4.2 Heat Available for Transfer from SOFC 34

5.5 Design of Heat Exchanger 34

5.6 Design of Heat Recouperator 36

5.7 Temperature and Pressure Variations along Guideway Heating System 37

Chapter 6. Analysis of Hybrid Guideway Heating System 39

6.1 Heat Supplied through Hybrid Guideway Heating System 39

6.2 Detailed Description of Hybrid Guideway Heating System 39

6.2.1 Guideway Fluid 39

6.2.2 Natural Gas Heater 40

6.2.3 SOFC Heater 40

6.2.4 Derivation of Heat Output from Hybrid Guideway Heating 40 System

6.3 Analysis of Hybrid Heating System 42

6.4 Heat Generated and Fuel Used 52 
6.5 Emissions Produced in Hybrid Heating System 53

6.6 Limitations for Hybrid Heating System 54

Chapter 7. Economic Justification $\quad 55$

7.1 Fixed and Variable Costs in Hybrid Heating System 55

7.2 Use of Commercial Fuel Cell in Hybrid Heating System 58

7.3 Cost Savings in Hybrid Heating System 59

7.3.1 Savings due to Fuel Cell Generated Electricity and Heat 59

7.3.2 Structural Savings in Hybrid Heating System 59

7.3.3 Net Cost Savings in Hybrid Heating System 60

7.4 Cost Comparison of Existing and Hybrid Heating Systems 60

7.5 Cost of Hydrogen and Natural Gas in Hybrid Heating System 62

7.6 Payback Period for Hybrid Heating System 63

$\begin{array}{ll}\text { Chapter 8. Results and Discussion } & 65\end{array}$

8.1 Results from Comparison of Existing and Hybrid Heating Systems 65

8.1.1 Hybrid Heating System using Hydrogen FC 65

8.1.2 Hybrid Heating System using Commercial FC 65

$\begin{array}{lll}8.2 \text { Conclusions } & 67\end{array}$

Chapter 9. Contributions and Accomplishments 69

9.1 Contributions and Accomplishments $\quad 69$

$\begin{array}{lll}9.2 & \text { Recommendations for Future Work } & 70\end{array}$

$\begin{array}{ll}\text { REFERENCES } & 71\end{array}$

APPENDIX A. Fuel Cell as Combined Heat and Power (CHP) Source 74

$\begin{array}{lll}\text { A.1 Introduction } & 74\end{array}$

A.2 Fuel Cell as a Combined Heat and Power (CHP) Source 75

APPENDIX B. Heat Produced from Solid Oxide Fuel Cell 77

$\begin{array}{lll}\text { B.1 Solid Oxide Fuel Cell } & 77\end{array}$ 
B.2 Chemical Equations Associated with SOFC 77

B.3 Equations for Heat Produced in Reforming and Shifting Reactions 78

$\begin{array}{ll}\text { B.4 Characteristics of SOFC } & 79\end{array}$

APPENDIX C. Fuel Cell as an Auxiliary Power Unit in Sleeper Trucks $\quad 81$

APPENDIX D. Data Provided by M-PRT 83

APPENDIX E. Historical Utility Costs for M-PRT 85

APPROVAL OF ADVISORY AND EXAMINING COMMITTEE 86 


\section{LIST OF FIGURES}

Figure 1.1 Guideway Connecting Different Stations in M-PRT System 1

Figure 3.1 Projected Natural Gas Consumption, 1990 - 2030 (in trillion cubic 11 feet)

Figure 3.2 Projected Natural Gas Supply 1990 - 2030 (in trillion cubic feet) 12

Figure 3.3 Projected Increase in Price of Natural Gas (in 2005 dollars per 12 thousand cubic feet)

Figure 4.1 Block Diagram of Existing Heating System 17

Figure 4.2 Heat Required to Melt Precipitated Snow Every Month 20

Figure 4.3 Heat Flow Diagram in Existing Heating System 21

Figure 4.4 Graph of Increased Heat Requirement with Decrease in Temperature 27

Figure 4.5 NOx Emissions from Existing Heating System 28

Figure 5.1 Illustrative Diagram for Hybrid Guideway Heating System 30

Figure 5.2 Schematic of Hybrid Guideway Heating System 31

Figure 5.3 Block Diagram of Hybrid Heating System 32

Figure 5.4 Heat Available from SOFC 33

Figure 5.5 Temperature Change in Inlet and Outlet Flows due to Heat 35

Exchanger

Figure 5.6 Temperature Change in Inlet and Outlet Flows due to Heat 36

Recouperator

Figure 5.7 Temperature Variation along Guideway Heating System 37

Figure 5.8 Pressure Variation along Guideway Heating System 38

Figure 6.1 Heat Flow Diagram in Hybrid Heating System 41

Figure 6.2 Heat Produced by Natural Gas Burner and Fuel Cell for Hybrid 52

$\begin{array}{ll} & \text { Heating System } \\ \text { Figure 6.3 Natural Gas used in Existing and Hybrid Heating System } & 53\end{array}$ 
Figure 6.4 Emissions Produced in Existing and Hybrid Heating System 54

Figure 7.1 Cost Ratios vs. Cost of Hydrogen 62

Figure 8.1 Cost Comparison of Existing and Hybrid Heating Systems 66

Figure 8.2 Net and Utility Cost Comparison $\quad 67$

$\begin{array}{lll}\text { Figure 8.3 Reduction in Emissions } & 68\end{array}$

Figure A.1 Conventional Power Generation and Combined Heat and Power 75 (CHP)

$\begin{array}{lll}\text { Figure B.1 Solid Oxide Fuel Cell } & 77\end{array}$

$\begin{array}{lll}\text { Figure B.2 Heat Generated vs Inlet Pressure } & 79\end{array}$

Figure B.3 Fuel Cell Temperature vs Fuel Flow Rate $\quad 79$

Figure C.1 SOFC Auxiliary Power Unit System Model 82 


\section{LIST OF TABLES}

Table 2.1 Comparison of Types of Fuel Cells 6

Table 3.1 Estimated Equipment Costs for Various Alternative Power 9 Equipment/Systems

Table 4.1 Data Provided for Boiler Plant 3 16

Table 4.2 Hours and Rate of Snowfall 18

Table 4.3 Calculations for Heat Required for Melting the Snow 19

Table 4.4 Heat Required to Melt Snow and to Keep Guideway at $43^{\circ} \mathrm{F}$

Table 5.1 Specifications of Fuel Cell Used 33

Table 6.1 Analysis of the Hybrid Heating System 44

Table 6.2 Heat Required and Cost of Fuel used in Existing and Hybrid Heating 51 Systems

Table 7.1 Assessment of M-PRT Guideway Heating Systems 56

Table 7.2 Savings in Hybrid Heating System 60

Table 7.3 Cost Comparison of Existing and Hybrid Heating Systems 61

Table 8.1 Comparison of Hybrid Heating System for varying power from 66 commercial FC

Table D.1 Data Provided by M-PRT $\quad 83$

Table E.1 Historical Utility Costs for M-PRT $\quad 85$ 


\section{Chapter 1}

\section{Introduction}

\subsection{Guideway Heating System for Morgantown Personal Rapid Transit}

The Morgantown Personal Rapid Transit (M-PRT) connects different stations in Morgantown, WV as shown in Figure 1.1, with vehicles running along a guideway. The

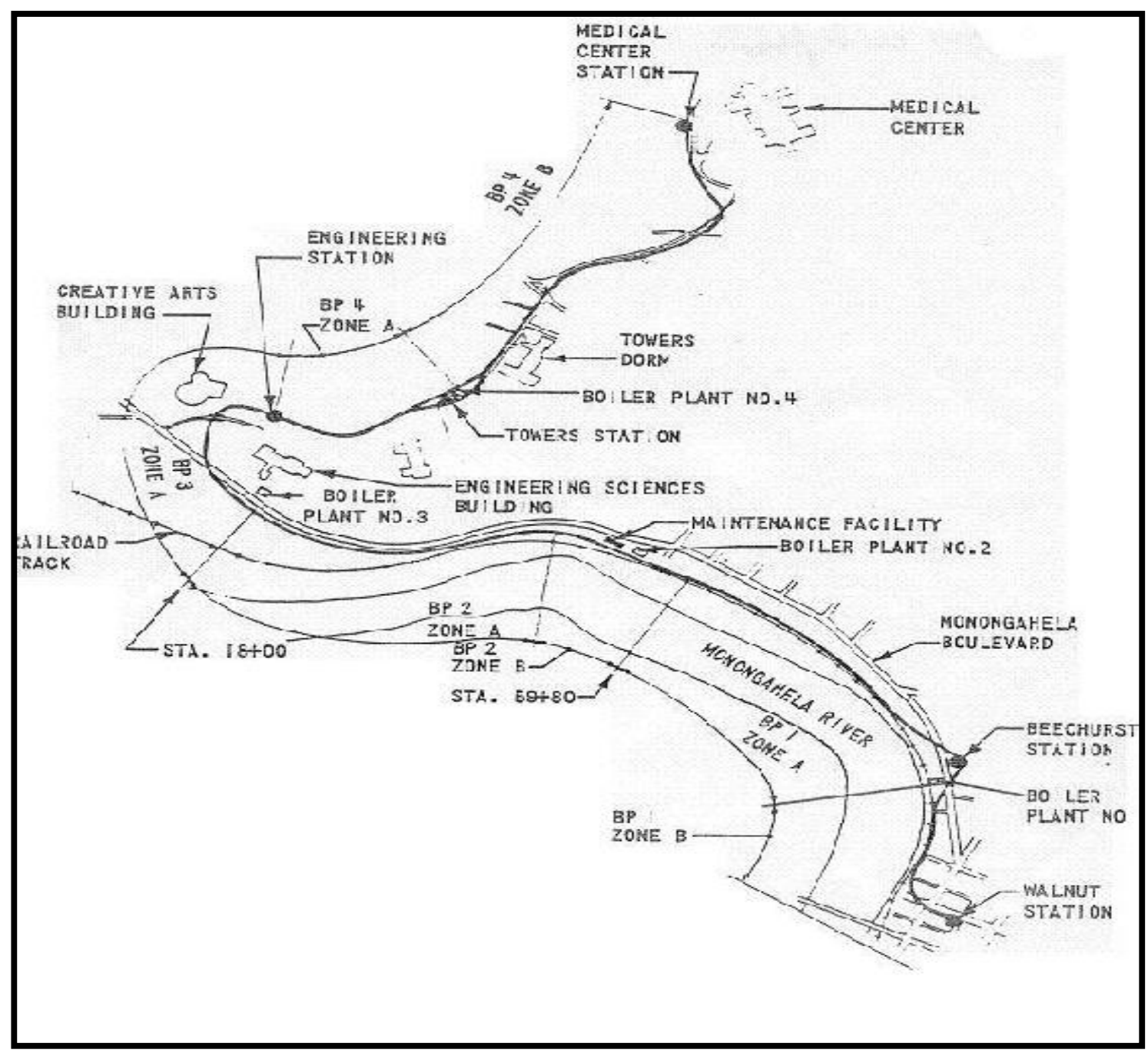

Figure 1.1: Guideway Connecting Different Stations in M-PRT System 
electric powered vehicles run along the guideway from one station to another automatically. For proper functioning of this M-PRT system, an ice-free guideway needs to be maintained. During precipitation conditions the guideway is heated to prevent any accumulation of snow, ice, etc. A 50\% ambitrol NTC liquid is heated in the existing MPRT system using a natural gas burner. The heated liquid is pumped through a pipe system that in part is located in the guideway.

There are four boiler plants that are supplying heat to the M-PRT system. Boiler Plant 3 (BP3) supplies the necessary heat for the guideway between Engineering Science Building and Creative Arts Center as shown in Figure 1.1. The length of guideway heated by BP3 is $2950 \mathrm{ft}$. A typical cross section of guideway is $10 \mathrm{ft}$ wide. But there are places in the guideway where the cross section is more than $10 \mathrm{ft}$ and thus making the total surface area heated by BP3 as $38,600 \mathrm{ft}^{2}$. The area heated by BP3 is about $20 \%$ of the total guideway in M-PRT system.

During extremely cold weather and when it snows, more heat should be supplied to compensate for the peak heat required for the guideway. The natural gas heating can become expensive to operate for maintaining an ice-free guideway. Several energy sources that could supply heat are fossil fuel combustions, solar heaters, electric heaters and fuel cells. But most of these sources have exhaust gases, which pollute the atmosphere. The fuel cell is a new technology of producing heat through the chemical oxidation of fuel. The electrochemical reaction that takes place inside the fuel cell releases high amount of usable heat.

\subsection{Fuel Cell as Source of Heat}

In this study, a fuel cell was specified as the source of heat. A fuel cell is a power generation unit that produces heat and electricity without combustion (Appendix A). Chemical energy is converted into heat and electricity when hydrogen is combined with oxygen from the air. Water is a by-product.

A fuel cell is unique in its potential for this M-PRT application as a source of heat. It is cleaner than the ordinary combustion processes as it extracts energy from a fuel chemically rather than from burning. It curbs emissions from the greenhouse gas carbon dioxide. A fuel cell is noise-free and has the advantage of using multiple types of fuel 
like hydrogen, natural gas and diesel for energy generation. It is portable and also produces electricity besides heat, which can improve the total efficiency of the fuel cell (Appendix B). Based on these different advantages the fuel cell is an attractive source of heat for the M-PRT guideway heating system.

\subsection{Technology Applications for M-PRT by FTA and WVU}

Federal Transit Administration (FTA) and West Virginia University (WVU) are working on evaluating fuel cell and its applications for M-PRT system. The Federal Grant FTA-WV-26-7001 was supporting a research in evaluating fuel cell and its technology for M-PRT [24]. The first phase of this project resulted in performing an extensive energy assessment of the M-PRT system [25]. The analysis included a breakdown of the utility costs for the major sub-systems in M-PRT. The main purpose of this analysis is to identify opportunities for reducing utility costs and energy consumption by implementing projects that will modernize existing equipment and systems.

This thesis contributes towards continuing the efforts of the FTA project into Phase 2. A fuel cell is identified as an alternate source for reducing energy consumption and utility costs for M-PRT.

\subsection{Research Objective}

The objective of this thesis is to design and analyze a hybrid guideway heating system using a Solid Oxide Fuel Cell (SOFC) as one of the sources of heat to warm the guideway in M-PRT. The SOFC, being a high temperature fuel cell, can provide the heat required for this application. The resultant hybrid guideway heating system shall be designed to be more cost effective and produce lower emissions than the existing heating system.

The principle involved with the hybrid guideway heating system is that a fuel cell is used as the primary source of heat to maintain the guideway at an operating temperature of $43^{\circ} \mathrm{F}$ always irrespective of snowfall. In addition to the fuel cell, a natural gas burner is also used as a secondary source of heat to melt the precipitated snow, as required. A hybrid heating system is to be designed and the energy and cost calculations are to be 
performed to determine the cost effectiveness of the system. A thermodynamic analysis is to be done to compute the size of fuel cell required and determine the maximum power required from hybrid heating system. 


\section{Chapter 2}

\section{Solid Oxide Fuel Cell as a Source of Heat}

\subsection{Performance Comparison of Different Fuel Cells}

High heat output and low emissions were the major reasons for considering the fuel cell as a source of heat. Fuel cells are best suited to produce supplemental energy based on factors such as efficiency and eco-friendliness. The noise levels are practically zero. Thermal performance evaluation of a fuel cell is dependent on the type and make of fuel cell. Different manufacturers have different topologies and to assess the performance, those specific design details may be required. However, in a typical thermal performance, parameters such as start-up time, fuel feed rate, catalyst, operating temperature and electrolyte material have a good impact [7].

There are six different types of fuel cells producing heat, which include

1. Alkaline Electrolyte Fuel Cell (AFC)

2. Proton Exchange Membrane Fuel Cell (PEMFC)

3. Direct Methanol Fuel Cell (DMFC)

4. Phosphoric Acid Fuel Cell (PAFC)

5. Molten Carbonate Fuel Cell (MCFC)

6. Solid Oxide Fuel Cell (SOFC)

A comparison of these fuel cells is shown in Table 2.1.

\subsection{High Temperature Fuel Cells}

Of the above six fuel cells, the PAFC, MCFC and SOFC are high temperature fuel cells. High temperature fuel cells are those that operate at temperatures higher than 1000 F. The operating temperature of these fuel cells reduces its open circuit voltage. At these temperatures the theoretical maximum efficiency of the fuel cell is also less than that of a heat engine [1]. It might, therefore, seem unwise to operate the fuel cells at 
Table 2.1: Comparison of Types of Fuel Cells [1]

\begin{tabular}{|c|c|c|c|c|c|c|}
\hline & AFC & PEMFC & DMFC & PAFC & MCFC & SOFC \\
\hline Electrolyte & $\begin{array}{l}\text { KOH absorbed } \\
\text { in asbestos } \\
\text { (Liquid) }\end{array}$ & $\begin{array}{c}\text { Fluorinated } \\
\text { Sulphonic Acid } \\
\text { (Solid) }\end{array}$ & & $\begin{array}{l}\text { Phosphoric } \\
\text { Acid in SiC } \\
\text { (Liquid) }\end{array}$ & $\begin{array}{c}\text { Sodium or Mg } \\
\text { Carbonates }\end{array}$ & $\begin{array}{l}\text { Calcium or } \\
\text { Zirconium } \\
\text { Oxides }\end{array}$ \\
\hline $\begin{array}{l}\text { Operating } \\
\text { Temperature }\end{array}$ & $200^{\circ} \mathrm{F}-380^{\circ} \mathrm{F}$ & $150^{\circ} \mathrm{F}-200^{\circ} \mathrm{F}$ & $80^{\circ} \mathrm{F}-150^{\circ} \mathrm{F}$ & $450^{\circ} \mathrm{F}$ & $1200^{\circ} \mathrm{F}$ & $1550^{\circ} \mathrm{F}-1850^{\circ} \mathrm{F}$ \\
\hline Mobile Ion & $\mathrm{OH}^{-}$ & $\mathrm{H}^{+}$ & $\mathrm{H}^{+}$ & $\mathrm{H}^{+}$ & $\mathrm{CO}_{3}{ }^{2-}$ & $\mathrm{O}^{2-}$ \\
\hline Catalyst & $\mathrm{Ni}, \mathrm{Ag}$ & $\mathrm{Pt}$ & $\mathrm{Pt} / \mathrm{Ru}$ & $\mathrm{Pt}$ & $\begin{array}{c}\text { Ni (anode) } \\
\text { Nickel Oxide } \\
\text { (Cathode) }\end{array}$ & $\begin{array}{c}\mathrm{Co}-\mathrm{ZrO}_{2} \mathrm{Ni}- \\
\mathrm{ZrO}_{2} \text { (anode) } \\
\mathrm{Sr}-\text { doped } \\
\mathrm{LaMnO}_{3} \\
\text { (Cathode) }\end{array}$ \\
\hline Efficiency & $70 \%$ & $40 \%-50 \%$ & & $40 \%-80 \%$ & $60 \%-80 \%$ & $60 \%$ \\
\hline Applications & $\begin{array}{c}\text { Used in Space } \\
\text { Vehicles }\end{array}$ & $\begin{array}{l}\text { *Vehicles and } \\
\text { mobile } \\
\text { application. } \\
\text { *Lower power } \\
\text { CHP systems. }\end{array}$ & $\begin{array}{c}\text { *Portable } \\
\text { electronic } \\
\text { systems of low } \\
\text { power. }\end{array}$ & $\begin{array}{c}\text { *Large } \\
\text { numbers of } 200 \\
\mathrm{~kW} \text { CHP } \\
\text { systems in use. }\end{array}$ & $\begin{array}{c}\text { *Large scale } \\
\text { CHP systems. } \\
\text { *Upto MW } \\
\text { capacity. }\end{array}$ & $\begin{array}{l}\text { *For all sizes of } \\
\text { CHP systems. } \\
\text { *From } 2 \mathrm{~kW} \text { to } \\
\text { multi MW } \\
\text { capacity. }\end{array}$ \\
\hline
\end{tabular}


these temperatures. But these problems are outweighed by the advantages of high temperature. The high temperature makes the electrochemical reaction proceed quickly. The high temperature exhaust gases (including water vapour) generated is a valuable source of heat. Thus, these fuel cells are not only considered as source of electricity, but they must be thought of as an integral part of a complete fuel utilizing and heat generating system.

\subsection{Advantages of SOFC}

The seven major advantages of SOFC in general and in comparison with other fuel cells are as follows:

1. The SOFC is a solid-state device that uses an oxide ion-conducting ceramic material as the electrolyte and it is more advantageous from the point of view of mechanical simplicity.

2. The SOFC is very flexible in the way it can be made, and its possible size [3].

3. The SOFC can be made from a range of different materials, with different operating temperatures, from about $1550^{\circ} \mathrm{F}$ to $1850^{\circ} \mathrm{F}$.

4. The high temperature gases leaving the SOFC carry large amount of heat energy. And, as the heat energy is obtained in exhaust gases, it can be transferred to the guideway fluid through a heat recouperator.

5. The SOFC is simpler in concept than all the other fuel cell system, as only two phases (gas and solid) are required [1].

6. Due to high operating temperature the use of precious metals as electro catalysts is eliminated.

7. Low noise levels and less undesired gases are released.

\subsection{Disadvantages of SOFC}

Though SOFC had many advantages, it has at least three disadvantages, which are as follows: 
1. The SOFC requires the development of suitable materials that have the required conductivity and remain solid at high temperatures. This requires expensive materials of construction [4].

2. The selected materials must be dense to prevent mixing of the fuel and oxidant gases.

3. An excess of sulfur in the fuel used in SOFC decreases its performance [7].

\subsection{Reason for Choosing SOFC}

The SOFC was chosen to provide heat to the guideway in the M-PRT system. The primary reason for choosing SOFC is because it is a high temperature fuel cell. The hybrid heating system defined in this thesis needs a suitable source of energy that releases high amounts of heat and is eco-friendly. Hence, the SOFC is the best choice as it releases high heat at $1850^{\circ} \mathrm{F}$. 


\section{Chapter 3}

\section{Project Statement and Approach}

\subsection{Phase 1 of FTA Project}

As a part of Phase 1 of FTA project, FTA and WVU have performed an energy assessment that would detail where, when and how energy was being used to determine the cost breakdown for the major subsystems of M-PRT. The energy consumption characteristics of the equipments and systems of M-PRT were observed and analyzed. The energy assessment helped understand the system, operations, components and operational cost. This information is important to understand how utility costs impact the system's operation and how a comprehensive energy management and modernization program may impact the system.

Table 3.1: Estimated Equipment Costs for Various Alternative Power Equipment/Systems [25]

\begin{tabular}{|c|c|}
\hline System & Estimated Cost per kW \\
\hline Emergency Generator & $\$ 250$ \\
\hline Cogeneration System & $\$ 1,100$ \\
\hline Microturbines & $\$ 1,200$ \\
\hline Fuel Cell & $\$ 3,700$ \\
\hline Wind Power & $\$ 1,300$ \\
\hline Photovoltaic & $\$ 7,200$ \\
\hline
\end{tabular}

During the evaluation of utility costs, natural gas was observed to be the most volatile utility for the M-PRT in consumption, since it is impacted by weather. Natural gas is predominantly used as a fuel for Boiler Plants which supply heat to the guideway during winter and snowing times. There were several alternative power equipment/systems suggested to improve the energy efficiency of M-PRT. The systems and their estimated cost per $\mathrm{kW}$ are mentioned in Table 3.1. 


\subsection{Goal for Project}

The major goals or objectives for this project are to

- Design a more efficient guideway heating system for M-PRT that

- Meet the peak load requirement during extreme cold weather conditions.

- Be cost effective than the existing heating system.

- Produce lower emissions than existing heating system and prove to be ecofriendly.

- Create a possibility of using an alternate fuel in hybrid heating system.

- Perform a thermodynamic analysis for the existing and hybrid guideway heating systems.

- Determine the size of fuel cell and maximum power required from hybrid heating system.

- Analyze the hybrid heating system to explain when and how the hybrid heating system can be effective for M-PRT than the existing heating system.

The hybrid heating system designed for the M-PRT satisfies all the requirements for this project and uses fuel cell as a primary source of heat that generates low undesired emissions. A fuel cell is used as a source of heat to maintain the guideway at an operating temperature throughout the year. This prevents any possible damage that the concrete may be subjected to during cold climates.

\subsection{Natural Gas in Existing Guideway Heating System}

The existing guideway heating system for the M-PRT uses a natural gas burner to provide the required heat for the guideway fluid. The natural gas used can become expensive when large quantities are needed. The price of the natural gas increases with the increase in its demand. Moreover, there is a projected increase in natural gas prices in the coming years. Energy Information Administration (EIA), in its latest release of the Annual Energy Outlook 2007 [9], forecasts higher natural gas consumption and higher natural gas prices than prior year's long-run projections. EIA has also reevaluated its expectations about the future role of natural gas in energy markets, the economics of natural gas exploration and development, and future natural gas price trends. Hence, 
there is a need for an alternate source of heat to meet the peak heat required for the guideway heating system in M-PRT. If the existing heating system is used then the MPRT system is required to pay a higher price for natural gas during the increased demand conditions.

\subsection{Difficulty with Existing Guideway Heating System}

The existing guideway heating system for the M-PRT has an activation period of $6 \%$ of winter and $2 \%$ in a year. Thus, the heater will be needed for an average of 170 hours a year. During the activation period, there is a peak heat requirement for $2 \%$ of winter. The ambient temperature goes below $15^{\circ} \mathrm{F}$ during this $2 \%$ of winter. There is a considerable heat lost due to the high difference between the guideway fluid temperature and ambient temperature. Hence, the ambient temperature plays a major role in the heat requirement.

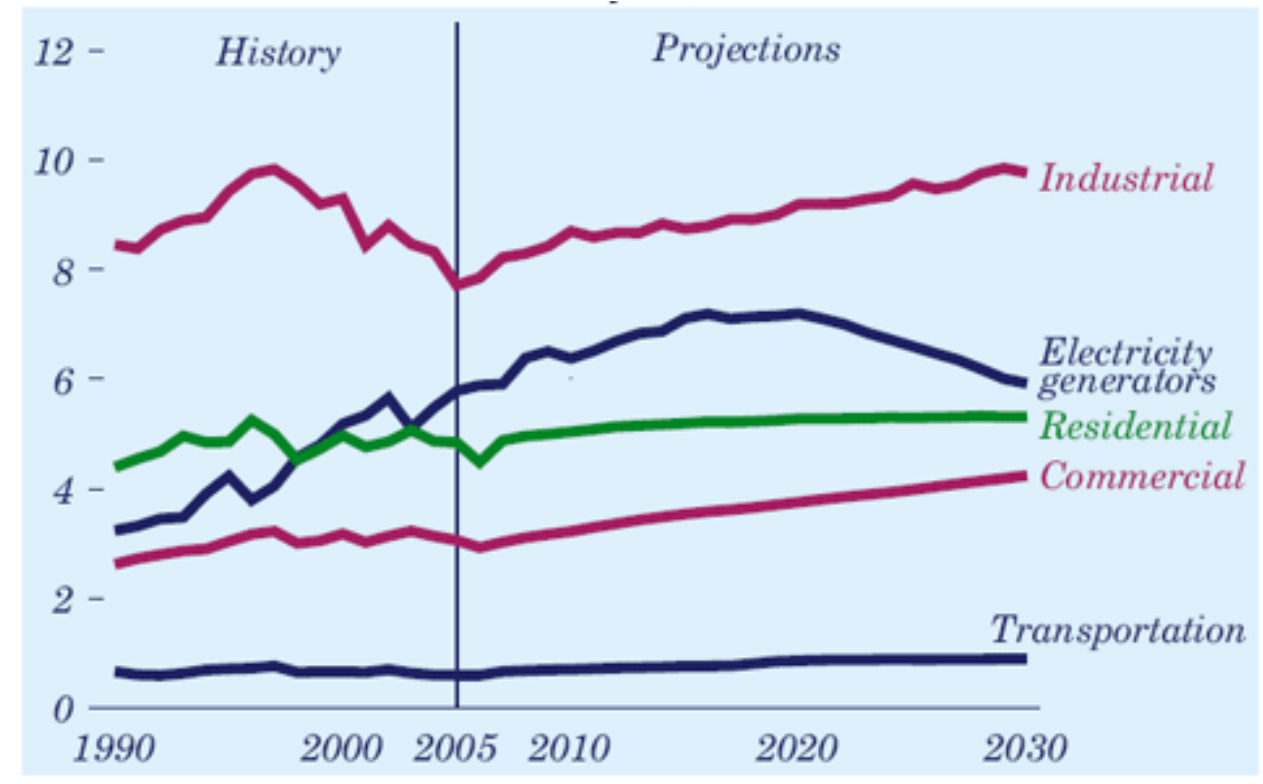

Figure 3.1: Projected Natural Gas Consumption, 1990 - 2030 (in trillion cubic feet)

The natural gas consumption in various sectors like Industrial, Electrical, Residential, Commercial and Transportation has been increasing every year. Total natural gas consumption is projected to grow to 26.1 trillion cubic feet in 2030 from 22 trillion cubic feet in 2005 as shown in EIA report [9]. On the other hand, the natural gas production declines in the AEO2007 reference case from 6.4 trillion cubic feet in 2005 to 4.9 trillion 
cubic feet in 2030. Hence, the projected consumption in natural gas is increasing and the projected supply is decreasing as mentioned in Figure 3.1 and Figure 3.2.

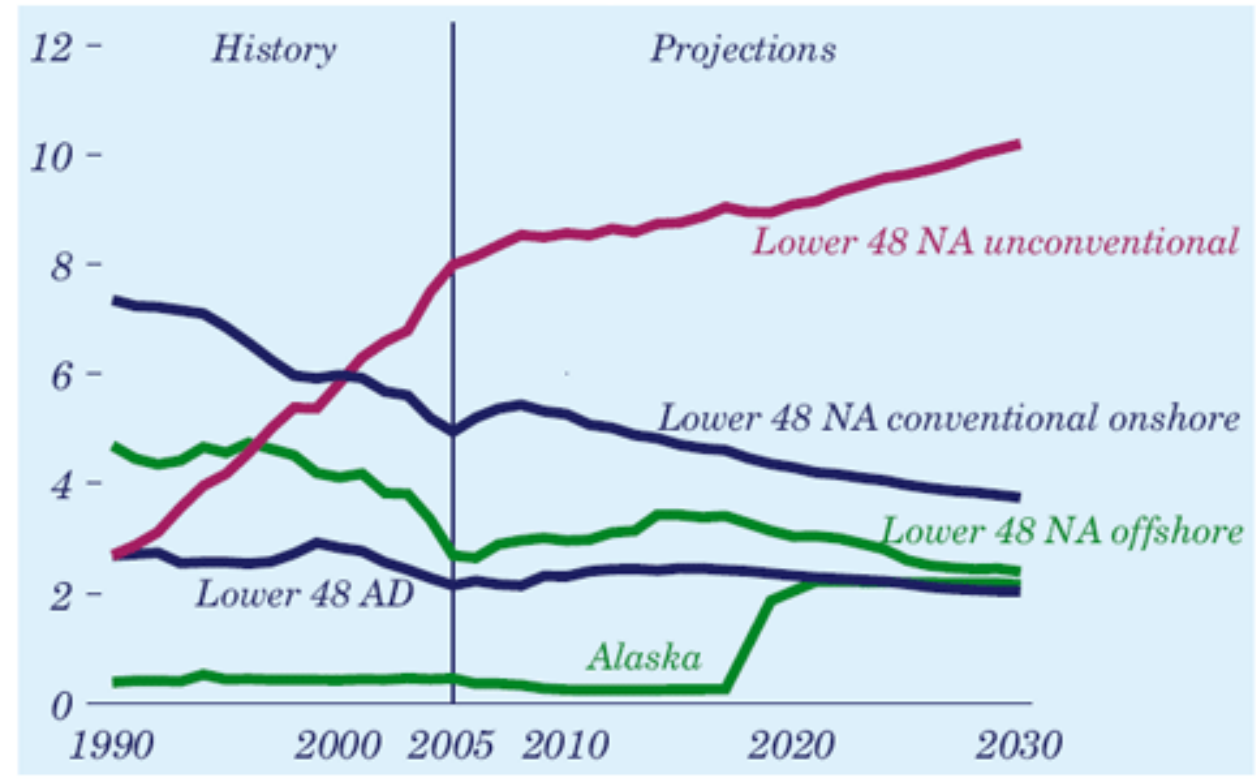

Figure 3.2: Projected Natural Gas Supply 1990 - 2030 (in trillion cubic feet) [9]

On one hand, when the production of natural gas decreases in the subsequent years, the forecasted price of the gas is also increasing from the reports released by the department

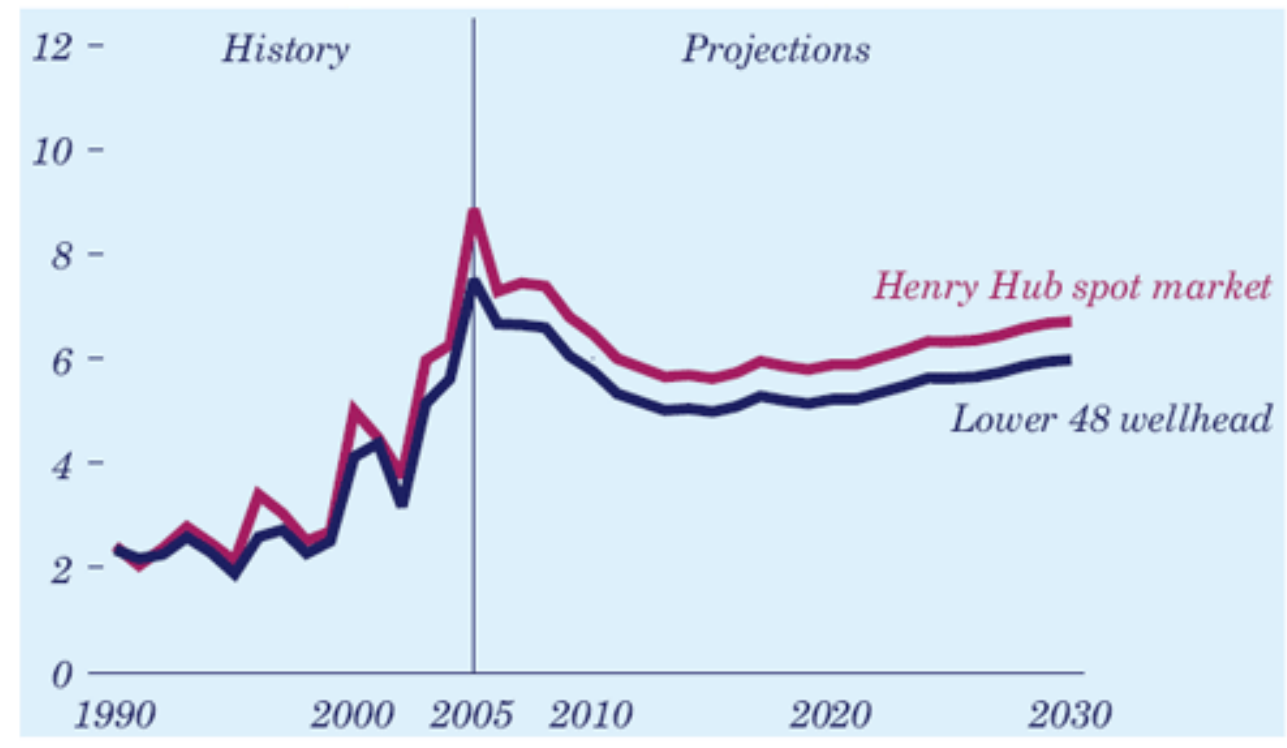

Figure 3.3: Projected Increase in Price of Natural Gas (in 2005 dollars per thousand cubic feet) [9] 
of energy. Average natural gas price is projected to increase from $\$ 5$ per thousand cubic feet in 2005 to $\$ 6.33$ per thousand cubic feet in 2030 [9] (See Figure 3.3). During the peak heat requirement, there is additional increase in the price of natural gas along with the increase in the demand for natural gas.

Figure 3.3 shows that the price of the natural gas increases gradually in the subsequent years. Hence, there is a serious requirement for an alternative source of heat.

\subsection{Approach for Hybrid Heating System}

To overcome the difficulty with the existing system, a hybrid heating system is being proposed which can suitably accommodate for the additional heat required during extreme climate conditions. The hybrid heating system consists of fuel cell and natural

gas burner. Fuel cell uses hydrogen as a fuel, which generates heat through chemical reaction. This process of heat generation is cleaner and highly eco-friendly as the fuel cell heater has low emissions. The natural gas heater, on the other hand, releases oxides of nitrogen that are harmful and pollute the atmosphere. The heat supplied by fuel cell is transferred to the guideway when required and for all other times the heat can be sold for other heating purposes. Fuel cell also generates electricity which increases the cost effectiveness of the system.

From the data provided by M-PRT, the heat required for maintaining the guideway at operating temperature and melting the snow was calculated. The heat required from fuel cell and gas burner was also calculated. Thus, a hybrid heating system is designed and analyzed in the subsequent chapters to explain the cost effectiveness.

\subsection{List of Assumptions}

There are few assumptions made in the analysis of this existing heating system and hybrid heating system. The assumptions made are listed below:

1. Exhaust gases account for $15 \%$ of heat produced from the natural gas burner.

2. The efficiency of natural gas burner is $87 \%$ and efficiency of heat exchanger and heat recouperator is $92 \%$.

3. Heat utilized by the guideway is $60 \%$ of the heat supplied to the guideway. 
4. Heat re-circulating back into the heat exchanger is $60 \%$ of heat coming out of guideway.

5. Fuel cell has a heat generation efficiency of $36 \%$.

6. Fuel cell has an electrical efficiency of $40 \%$.

7. The cost of operation to reduce emissions through Selective Catalytic Reduction (SCR) is $\$ 18.75 / \mathrm{MBtu}$.

8. Heat capacity of natural gas and hydrogen are constant throughout irrespective of the operation of fuel cell and natural gas burner.

9. The fuel cell is assumed to provide constant heat during its operation.

10. Hydrogen used for fuel cell is in a compressed form and it is stored safely.

11. The price of hydrogen is considered constant for any ambient temperature. 


\section{Chapter 4}

\section{Thermodynamic Analysis of Existing Guideway Heating System}

\subsection{Purpose of Guideway Heating System}

The M-PRT system is the most comfortable conveyance for travel at West Virginia University. The system has vehicles that run on a guideway connecting all the important places of the town and university campus. The vehicles face a comfortable situation during normal climate conditions. But the vehicles find a hazardous situation when it snows. There is a good possibility of skidding or overturning of the vehicles. Hence, the system should be designed to maintain an ice-free guideway all through the year. For this purpose, a heating system is provided that melts the ice and snow from the guideway. During peak conditions, due to distribution, there is a considerable loss of heat, which increases the demand for an additional supply of heat.

\subsection{Guideway Heating System in Morgantown Personal Rapid Transit (M-PRT)}

The guideway heating system in M-PRT is provided to maintain snow and ice-free guideway. The system is designed to provide 205 Btu (60 watt equivalency) input per square foot of guideway surface. The input rate is based on a one inch per hour precipitation (snow rate). It takes one and half-hour for the concrete slab to reach the temperature of $32^{\circ} \mathrm{F}$ from an initial temperature of $0^{\circ} \mathrm{F}$. The heating system uses natural gas boilers and heat exchangers to heat the heating fluid (a 50\% Ambitrol NTC), which circulates underneath the guideway of the M-PRT. Natural gas is used as a fuel for heating purposes in the boilers.

Ambitrol is a good heat transfer fluid. It is specially formulated to meet the severe and fluctuating year-round operating requirements of large, heavy-duty applications. These heating fluids include either ethylene glycol or propylene glycol-based products. The primary difference between ethylene glycol and propylene glycol based coolants is viscosity. At temperatures below $0^{\circ} \mathrm{F}$, ethylene glycol is less viscous than propylene 
glycol [20]. But at temperatures above $0^{\circ} \mathrm{F}$, propylene glycol has lesser viscosity and hence it provides improved heat transfer efficiency.

Ambitrol CN is an ethylene glycol fluid and ambitrol NTC is a propylene glycol fluid. Due to its low acute oral toxicity, ambitrol NTC are recommended for applications where incidental contact with drinking water or ground water is possible. Because of the abovementioned reasons, a $50 \%$ ambitrol NTC with $50 \%$ of water is used as a heating fluid in the guideway of the M-PRT system. Dow Chemical Company is the only national supplier that offers a choice of glycol-based fluids. The specific heat of ambitrol NTC fluid ranges from 0.86 to $0.9 \mathrm{Btu} / \mathrm{lb} .^{\circ} \mathrm{F}$ for a fluid temperature ranging from $100^{\circ} \mathrm{F}$ to $180^{\circ} \mathrm{F}[11]$.

\subsection{Thermodynamic Analysis of Existing Heating System}

As provided in the Energy Assessment Report by WVU and FTA, the guideway heating system is comprised of 17 boilers located in four boiler plants. These boiler plants supply the heating fluid throughout the M-PRT guideway operating at $140 \mathrm{~F}$. This is done to prevent snow and ice forming on the guideway. In most cases, snow and ice will not occur when the temperature is over $40^{\circ} \mathrm{F}$. Therefore, the boilers are shut off when the outside air temperature reaches $43^{\circ} \mathrm{F}$, which is the operating temperature that the guideway is designed to reach at the end of each heating section. Data is provided for the Boiler Plant 3 (BP3), which covers a certain area of the M-PRT system for providing the heating fluid. There are two boilers in BP3 and heat output from BP3 is given in Table 4.1. Hence, each boiler provides the required amount of heat to the system. The cost of replacing the boilers of this size is $\$ 65,000$ per boiler.

Table 4.1: Data Provided for Boiler Plant 3

\begin{tabular}{|c|c|}
\hline Number of boilers in BP3 & 2 \\
\hline Length of guideway heated by BP3 & $2950 \mathrm{ft}$ \\
\hline Total area of guideway surface heated by BP3 & $38,600 \mathrm{ft}^{2}$ \\
\hline Maximum heat input from each boiler & $6,000,000 \mathrm{Btu} / \mathrm{hr}$ \\
\hline Maximum heat output from each boiler & $4,800,000 \mathrm{Btu} / \mathrm{hr}$ \\
\hline Maximum heat output from BP3 & $9,600,000 \mathrm{Btu} / \mathrm{hr}$ \\
\hline
\end{tabular}


The boiler plant in existing heating system uses a natural gas burner to provide the heat required to the fluid passing through the heat exchanger. The heating fluid $(50 \%$ Ambitrol NTC) is circulated all through the guideway using a pipeline system. The large pipes are opened into a grid shaped section of smaller pipes that runs in the concrete of the guideway. The delivery pipe system that runs parallel to the guideway has a decreasing cross-section so as to maintain the pressure of the fluid almost constant through the pipeline as shown in Figure 5.7.

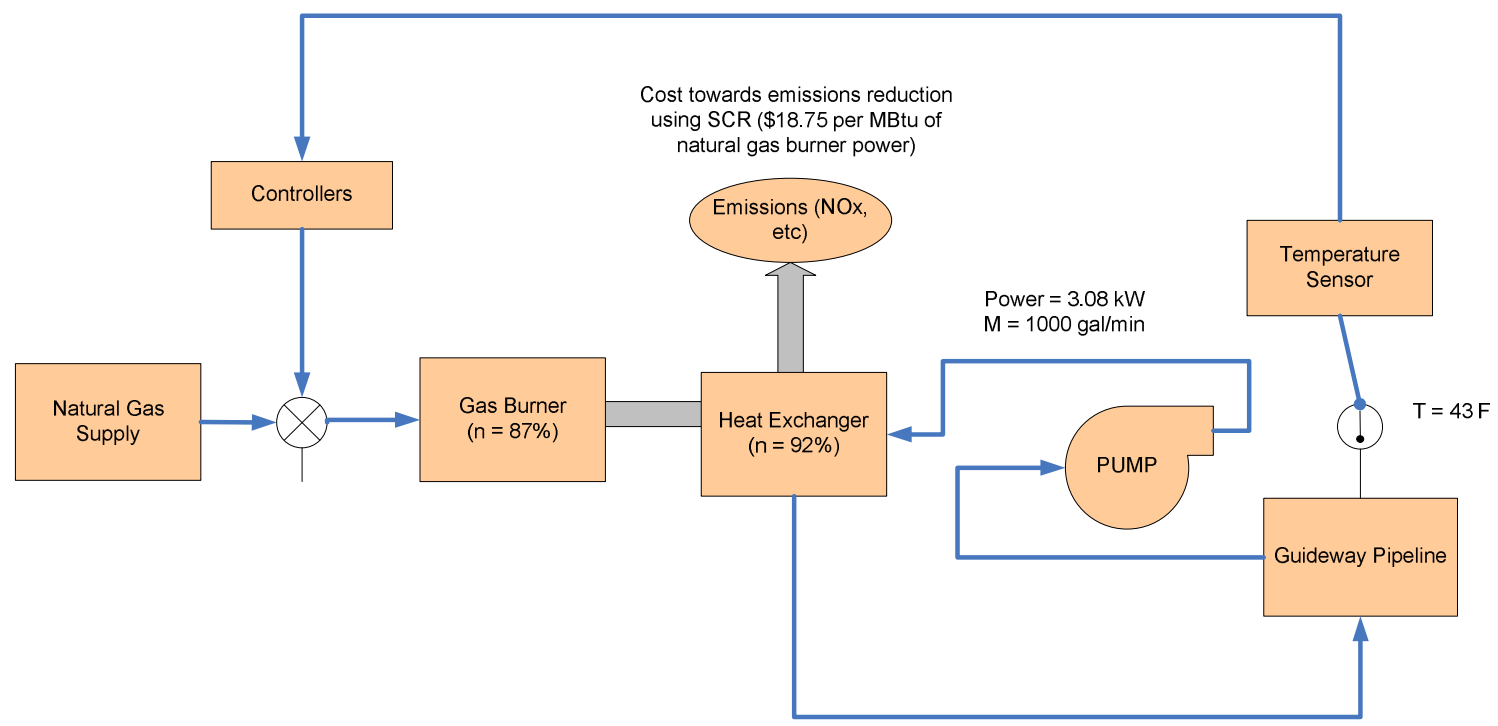

Figure 4.1: Block Diagram of Existing Heating System

The block diagram of the existing heating system is shown in Figure 4.1. The natural gas burner in the existing heating system is operated only during the hours of snowfall. The natural gas supply is controlled to provide the heat required for the guideway of the M-PRT. As shown in Table 4.1, the two gas burners in existing heating system provide a maximum of 9.6 million Btu of heat per hour. The flow rate in the pipes of the guideway heating system is around 1000 gallons per minute. The heating system is operated based on the changing climate conditions. When there is a precipitation, heat is supplied by the existing heating system. Operating the inlet flow of the natural gas, the required heat is supplied to the guideway. More natural gas is supplied when more heat is required and vice-versa. 


\subsubsection{Hours and Rate of Snowfall}

M-PRT experiences heavy snowfall during winter between November to March. The amount of snowfall varies every year. M-PRT has provided us with some real-time data which includes the ambient temperature, rate of snowfall and hours of snowfall for the period of December 2003 and March 2004. The average values are computed for those four months as shown in Table 4.2. Hence, M-PRT has experienced a snowfall for 170 hours out of a total 2976 hours. The total amount of snowfall is about 86 inches.

Table 4.2: Hours and Rate of Snowfall

\begin{tabular}{|c|c|c|c|c|c|}
\hline MONTH & $\begin{array}{c}\text { Average } \\
\text { ambient } \\
\text { temp }\end{array}$ & $\begin{array}{c}\text { Average } \\
\text { rate of } \\
\text { snowfall }\end{array}$ & $\begin{array}{c}\text { Hours of } \\
\text { snowfall in } \\
\text { each month }\end{array}$ & $\begin{array}{c}\text { Total } \\
\text { amount of } \\
\text { snowfall }\end{array}$ & $\begin{array}{c}\text { Total } \\
\text { number of } \\
\text { hours in } \\
\text { each month }\end{array}$ \\
\hline & $(\mathrm{F})$ & $(\mathrm{in} / \mathrm{hr})$ & $(\mathrm{hr})$ & $($ in. $)$ & $(\mathrm{hr})$ \\
\hline December & 27.3 & 0.42 & 15.4 & 6.5 & 792 \\
\hline January & 22.5 & 0.57 & 129.2 & 73.6 & 744 \\
\hline February & 24.4 & 0.35 & 11.1 & 3.9 & 696 \\
\hline March & 31.8 & 0.38 & 14.0 & 1.5 & 744 \\
\hline TOTAL & & & $\mathbf{1 6 9 . 7}$ & & $\mathbf{2 9 7 6}$ \\
\hline
\end{tabular}

\subsubsection{Heat Required by Guideway}

Heat required $\left(\mathrm{Q}_{\mathrm{c}}\right)$ to maintain the guideway at $43^{\circ} \mathrm{F}$ is

$$
\mathrm{Q}_{\mathrm{c}}=\mathrm{h}\left(43-\mathrm{t}_{\mathrm{a}}\right)
$$

Heat required $\left(\mathrm{Q}_{\mathrm{m}}\right)$ to melt the snow

$$
\mathrm{Q}_{\mathrm{m}}=\mathrm{Q}_{\mathrm{et}}+\mathrm{Q}_{\mathrm{f}}
$$

where

$\mathrm{Q}_{\mathrm{et}}=$ Sensible heat transferred to the snow $\left(\mathrm{Btu} / \mathrm{hr} . \mathrm{ft}^{2}\right)$

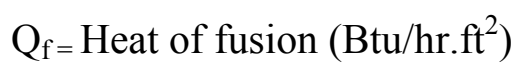

The sensible heat $\left(\mathrm{Q}_{e t}\right)$ to raise the temperature of snow to $32^{\circ} \mathrm{F}$ is

$$
Q_{e t}=\frac{s C_{p} D\left(32-t_{a}\right)}{C_{1}}
$$

The heat of fusion $\left(\mathrm{Q}_{\mathrm{f}}\right)$ to melt the snow is 


$$
Q_{f}=\frac{s h_{f} D}{C_{1}}
$$

Total heat required to maintain the guideway at $43^{\circ} \mathrm{F}$ and to melt snow is given by:

$$
\mathrm{Q}_{\mathrm{GW} / \text { loss }}=\mathrm{Q}_{\mathrm{c}}+\mathrm{Q}_{\mathrm{m}}
$$

\section{Nomenclature:}

$$
\begin{array}{lll}
\text { Variables } & \mathrm{S} & \text { rate of snow fall }(\mathrm{in} / \mathrm{hr}) \\
& \mathrm{d} & \text { duration of snow fall }(\mathrm{hr}) \\
& \mathrm{t}_{\mathrm{a}} & \text { ambient temperature }\left({ }^{\circ} \mathrm{F}\right) \\
\text { Constants } & \mathrm{h}_{\mathrm{f}} & \text { heat of fusion of snow }(\mathrm{Btu} / \mathrm{lb})=144 \\
& \mathrm{D} & \text { density of snow }\left(\mathrm{lb} / \mathrm{ft}^{3}\right)=8.91 \\
\mathrm{~A} & \text { surface area of heated guideway }\left(\mathrm{ft}^{2}\right)=38,600 \\
\mathrm{C}_{1} & \text { conversion factor }(\mathrm{in} / \mathrm{ft})=12 \\
\mathrm{C}_{\mathrm{p}} & \text { specific heat of snow }\left(\mathrm{Btu} / \mathrm{lb}-{ }^{\circ} \mathrm{F}\right)=0.5 \\
\mathrm{~h} & \text { convection heat transfer coefficient for air }\left(\mathrm{Btu} / \mathrm{hr}-{ }^{\circ} \mathrm{F}-\mathrm{ft}^{2}\right)=14.3
\end{array}
$$

Table 4.3: Calculations for Heat Required for Melting the Snow [8]

\begin{tabular}{|c|c|c|c|c|c|}
\hline Month & $\begin{array}{c}\text { Average } \\
\text { Ambient } \\
\text { Temperature } \\
\left({ }^{\circ} \mathrm{F}\right)\end{array}$ & $\begin{array}{c}\text { Snowfall } \\
\text { Rate } \\
(\text { inches } / \mathrm{hr})\end{array}$ & $\begin{array}{c}\text { Sensible } \\
\text { Heat }\left(\mathrm{Q}_{\mathrm{et}}\right) \\
\text { Transferred } \\
\text { to Snow } \\
\left(\mathrm{Btu} / \mathrm{hr} . \mathrm{ft}^{2}\right)\end{array}$ & $\begin{array}{c}\text { Heat }\left(\mathrm{Q}_{\mathrm{f}}\right) \text { of } \\
\text { Fusion } \\
\left(\mathrm{Btu} / \mathrm{hr} . \mathrm{ft}^{2}\right)\end{array}$ & $\begin{array}{c}\text { Total Heat }\left(\mathrm{Q}_{\mathrm{m}}\right) \\
\text { to Melt } \\
\text { Snow }\left(\mathrm{Btu} / \mathrm{hr} . \mathrm{ft}^{2}\right)\end{array}$ \\
\hline Dec & 27.3 & 0.42 & 1.3 & 266 & 267 \\
\hline Jan & 22.5 & 0.57 & 87.3 & 2790 & 2880 \\
\hline Feb & 24.4 & 0.35 & 0.7 & 225 & 226 \\
\hline Mar & 31.8 & 0.38 & 1.4 & 247 & 248 \\
\hline
\end{tabular}

Table 4.3 explains the calculations for heat required for melting the snow. The sensible heat transferred to snow $\left(\mathrm{Q}_{\mathrm{et}}\right)$ and heat of fusion $\left(\mathrm{Q}_{\mathrm{f}}\right)$ is calculated using equations 4.3 and 4.4. The total heat to melt snow $\left(\mathrm{Q}_{\mathrm{m}}\right)$ is calculated for all the four months during the winter.

The heat required for melting precipitated snow increases with increase in snowfall. The heat required is directly proportional to the rate of snowfall and also to the decrease in ambient temperature. The sensible heat needed to melt the snow depends on the 
precipitation of snow. The above calculations are based on the assumption that the accumulated snow is melted after total precipitation. Hence, under those conditions, the total heat required to melt the snow varies from $226 \mathrm{Btu} / \mathrm{hr} . \mathrm{ft}^{2}$ to $2880 \mathrm{Btu} / \mathrm{hr}^{. \mathrm{ft}^{2}}$ as shown in Figure 4.2.

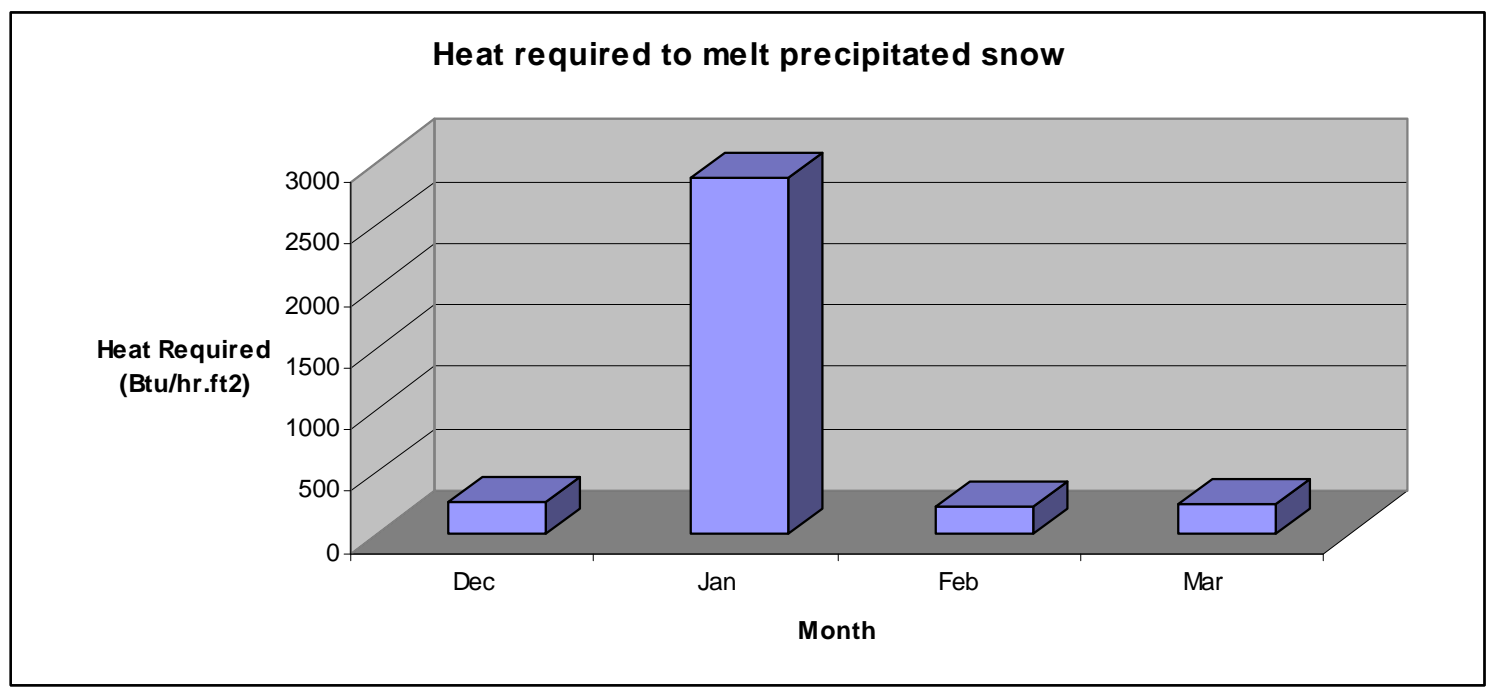

Figure 4.2: Heat Required to Melt Precipitated Snow Every Month

\subsubsection{Derivation of Heat Output from Existing Heating System}

Efficiency of gas burner and heat exchanger in existing guideway heating system are assumed to be $87 \%$ and $92 \%\left(=\eta_{1}\right)$ respectively. Guideway receives heat $\left(\mathrm{Q}_{\mathrm{GW} / \mathrm{in}}\right)$ via the heat exchanger as shown in Figure 4.3. It is assumed that $60 \%\left(=\eta_{2}\right)$ of the heat ( $\left.\mathrm{Q}_{\mathrm{GW} / \text { loss }}\right)$ supplied to the guideway will be utilized in melting snow as mentioned in equation 4.6.

Hence, $\mathrm{Q}_{\mathrm{GW} / \mathrm{loss}}=0.6 * \mathrm{Q}_{\mathrm{GW} / \mathrm{in}}$

Therefore, the remaining heat $\left(\mathrm{Q}_{\mathrm{GW} / \mathrm{out}}\right)$ is equal to $40 \%\left(=\eta_{3}\right)$ of heat supplied to the guideway as shown in equation 4.7 .

$$
\mathrm{Q}_{\mathrm{GW} / \text { out }}=0.4 * \mathrm{Q}_{\mathrm{GW} / \text { in }}
$$

It is assumed that $40 \%$ of remaining heat $\left(\mathrm{Q}_{\mathrm{GW} / \mathrm{out}}\right)$, will be lost to sides and bottom of the guideway and the rest will be the heat re-circulated $\left(\mathrm{Q}_{\mathrm{RC} / \mathrm{HE}}\right)$ back into the heat exchanger as mentioned in equation 4.8 .

$$
\mathrm{Q}_{\mathrm{RC} / \mathrm{HE}}=0.6 * \mathrm{Q}_{\mathrm{GW} / \text { out }}
$$


Efficiencies of the heat exchanger, guideway and sides \& pipes will be calculated as mentioned below using equations 4.6, 4.7 and 4.8.

Definitions: HE: $\eta_{1}=\left(\mathrm{Q}_{3}-\mathrm{Q}_{7}\right) / \mathrm{Q}_{1} ; \mathrm{GW}: \eta_{2}=\mathrm{Q}_{4} / \mathrm{Q}_{3} ; \mathrm{SP}: \eta_{3}=\mathrm{Q}_{6} / \mathrm{Q}_{5}$;

Energy Analyses: HE: $\mathrm{Q}_{3}=\mathrm{Q}_{7}+\eta_{1} \mathrm{Q}_{1} ; \mathrm{GW}: \mathrm{Q}_{5}=\mathrm{Q}_{3}-\mathrm{Q}_{4} ; \mathrm{SP}: \mathrm{Q}_{7}=\mathrm{Q}_{5}-\mathrm{Q}_{6}$;

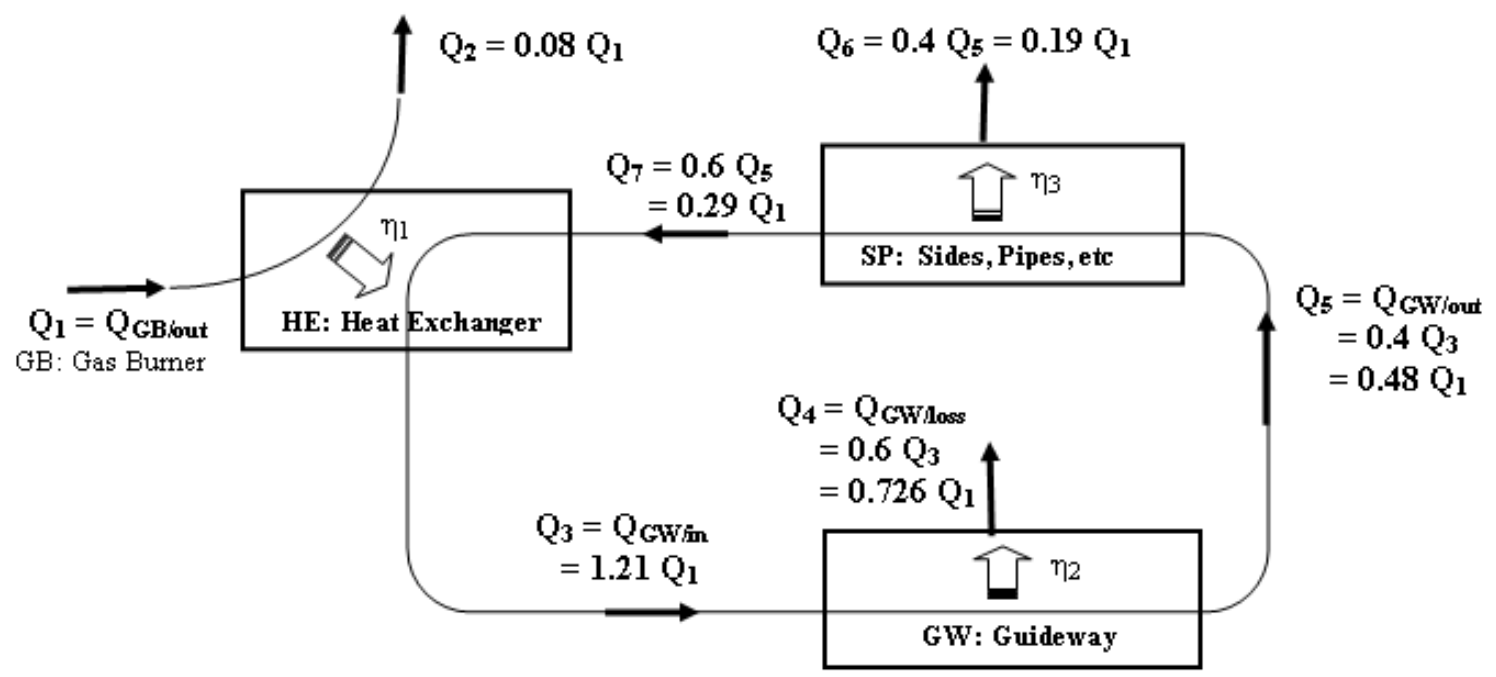

Figure 4.3: Heat Flow Diagram in Existing Heating System

Using the definitions and energy analyses for Heat Exchanger (HE), Guideway (GW) and Sides, Pipes, etc (SP), the following equations are obtained as

$$
\begin{aligned}
& \text { GW: } Q_{4}=\eta_{2} Q_{3} \\
& \text { and } Q_{5}=\left(1 / \eta_{2}-1\right) Q_{4} \\
& \text { HE: } Q_{3}=Q_{7}+\eta_{1} Q_{1} \\
& \text { SP: } Q_{7}=\left(1-\eta_{3}\right) Q_{5}
\end{aligned}
$$

Thus, using the four equations $4.9,4.10,4.11$ and 4.12 to solve for $\mathrm{Q}_{4}=f\left(\mathrm{Q}_{1}\right)$ yields

$$
\mathrm{Q}_{4}=\left(\eta_{1} \eta_{2}\right) /\left[1-\left(1-\eta_{2}\right)\left(1-\eta_{3}\right)\right] \mathrm{Q}_{1}
$$

Using the values for efficiencies, $\eta_{1}=0.92, \eta_{2}=0.6$, and $\eta_{3}=0.4$,

$$
\text { Then, } \mathrm{Q}_{4}=0.726 \mathrm{Q}_{1}
$$

\section{Nomenclature:}

$\mathrm{Q}_{1} \quad$ Heat output from gas burner $\left(\mathrm{Q}_{\mathrm{GB} / \mathrm{out}}\right)$

$\mathrm{Q}_{2} \quad$ Heat lost in heat exchanger

$\mathrm{Q}_{3} \quad$ Heat entering into the guideway $\left(\mathrm{Q}_{\mathrm{GW} / \mathrm{in}}\right)$ 
$\mathrm{Q}_{4} \quad$ Heat utilized by the guideway $\left(\mathrm{Q}_{\mathrm{GW} / \mathrm{loss}}\right)$

$\mathrm{Q}_{5} \quad$ Heat coming out of the guideway ( $\left.\mathrm{Q}_{\mathrm{GW} / \mathrm{out}}\right)$

$\mathrm{Q}_{6} \quad$ Heat lost to the sides, pipes, bottom etc of the guideway (= $\left.0.4 \mathrm{Q}_{5}\right)$

$\mathrm{Q}_{7} \quad$ Heat re-circulated back into the heat exchanger $\left(\mathrm{Q}_{\mathrm{RC} / \mathrm{HE}}=0.6 \mathrm{Q}_{5}\right)$

Equivalent power of heat from gas burner is calculated as:

$P=\left(Q_{G B / \text { out }} /\right.$ DeltaTime $\left./ 3.41\right) * 10^{3}$

where $3.41 * 10^{3}$ is the conversion factor from Btu/hr to $\mathrm{kW}$.

The heat capacity of natural gas is $1000 \mathrm{Btu} / \mathrm{ft}^{3}$ [9] and the average cost of natural gas is $\$ 5 / \mathrm{MBtu}$ (from 2005 dollars) [9]. The total cost of natural gas (C) required is calculated as

$$
\mathrm{C}=1.2 *\left(\mathrm{C}_{\mathrm{NG}}\right) *\left(\mathrm{Q}_{\mathrm{GB} / \text { out }}\right) /\left(\eta_{\mathrm{GB}}\right)
$$

where

$\mathrm{C}_{\mathrm{NG}}-$ Average cost of natural gas $(\$ 5 / \mathrm{MBtu})$

$\mathrm{Q}_{\mathrm{GB} / \text { out }}-$ Heat from natural gas burner (in MBtu)

$\eta_{\mathrm{GB}}-$ Efficiency of natural gas burner $(87 \%)$

The four columns in Table 4.4 named as calendar date, ambient temperature, time of snowfall and rate of snowfall are the data provided by M-PRT. The heat required by the natural gas burner to melt the snow and to maintain the guideway at a temperature of $43^{\circ} \mathrm{F}$ was calculated. These calculations are for the period during winter from December 2003 to March 2004 for M-PRT guideway heating system. Heat from natural gas burner is computed using equation 4.14. The cost of natural gas for producing the required heat is calculated using equation 4.16 .

Table 4.4: Heat Required to Melt Snow and to Keep Guideway at $43^{\circ} \mathrm{F}$

\begin{tabular}{|c|c|c|c|c|c|c|c|}
$\begin{array}{c}\text { Calendar } \\
\text { Date }\end{array}$ & $\begin{array}{c}\text { Ambient } \\
\text { Temp }\end{array}$ & $\begin{array}{c}\text { Time of } \\
\text { Snowfall }\end{array}$ & $\begin{array}{c}\text { Rate of } \\
\text { Snow } \\
\text { fall }\end{array}$ & $\begin{array}{c}\text { Heat to } \\
\text { Melt } \\
\text { Snow }\end{array}$ & $\begin{array}{c}\text { Convection } \\
\text { Heat Loss }\end{array}$ & $\begin{array}{c}\text { Heat } \\
\text { from Gas } \\
\text { Burner }\end{array}$ & $\begin{array}{c}\text { Cost of } \\
\text { Natural } \\
\text { Gas }\end{array}$ \\
\hline $11 / 29 / 2003$ & 26.9 & 18.8 & 0 & 0 & 0 & 0 & $\left(\begin{array}{c}\text { (in) } \\
\left({ }^{\circ} \mathrm{Fr}\right)\end{array}\right.$ \\
\hline & 29.0 & 5.2 & 0.47 & 10.2 & 40.1 & 69 & $\$ 477$ \\
\hline $11 / 30 / 2003$ & 29.0 & 24 & 0 & 0 & 0 & 0 & $\$ 0$ \\
\hline $12 / 1 / 2003$ & 29.0 & 24 & 0 & 0 & 0 & 0 & $\$ 0$ \\
\hline $12 / 2 / 2003$ & 29.0 & 24 & 0 & 0 & 0 & 0 & $\$ 0$ \\
\hline $12 / 3 / 2003$ & 29.0 & 24 & 0 & 0 & 0 & 0 & $\$ 0$ \\
\hline $12 / 4 / 2003$ & 29.0 & 24 & 0 & 0 & 0 & 0 & $\$ 0$ \\
\hline
\end{tabular}




\begin{tabular}{|c|c|c|c|c|c|c|c|}
\hline $12 / 5 / 2003$ & 26.9 & 17.4 & 0 & 0 & 0 & 0 & $\$ 0$ \\
\hline & 36.0 & 2.0 & 0.36 & 2.9 & 7.7 & 15 & $\$ 101$ \\
\hline & 35.0 & 3.3 & 0.39 & 5.2 & 14.5 & 27 & $\$ 188$ \\
\hline & 35.0 & 1.3 & 0.39 & 2.1 & 5.7 & 11 & $\$ 74$ \\
\hline \multirow[t]{3}{*}{$12 / 6 / 2003$} & 26.9 & 20.4 & 0 & 0 & 0 & 0 & $\$ 0$ \\
\hline & 34.0 & 2.4 & 0.40 & 3.9 & 11.9 & 22 & $\$ 150$ \\
\hline & 28.0 & 1.2 & 0.48 & 2.4 & 9.9 & 17 & $\$ 117$ \\
\hline $12 / 7 / 2003$ & 26.9 & 24 & 0 & 0 & 0 & 0 & $\$ 0$ \\
\hline $12 / 8 / 2003$ & 26.9 & 24 & 0 & 0 & 0 & 0 & $\$ 0$ \\
\hline $12 / 9 / 2003$ & 26.9 & 24 & 0 & 0 & 0 & 0 & $\$ 0$ \\
\hline $12 / 10 / 2003$ & 26.9 & 24 & 0 & 0 & 0 & 0 & $\$ 0$ \\
\hline $12 / 11 / 2003$ & 26.9 & 24 & 0 & 0 & 0 & 0 & $\$ 0$ \\
\hline $12 / 12 / 2003$ & 26.9 & 24 & 0 & 0 & 0 & 0 & $\$ 0$ \\
\hline $12 / 13 / 2003$ & 26.9 & 24 & 0 & 0 & 0 & 0 & $\$ 0$ \\
\hline $12 / 14 / 2003$ & 26.9 & 24 & 0 & 0 & 0 & 0 & $\$ 0$ \\
\hline $12 / 15 / 2003$ & 26.9 & 24 & 0 & 0 & 0 & 0 & $\$ 0$ \\
\hline $12 / 16 / 2003$ & 26.9 & 24 & 0 & 0 & 0 & 0 & $\$ 0$ \\
\hline $12 / 17 / 2003$ & 26.9 & 24 & 0 & 0 & 0 & 0 & $\$ 0$ \\
\hline $12 / 18 / 2003$ & 26.9 & 24 & 0 & 0 & 0 & 0 & $\$ 0$ \\
\hline $12 / 19 / 2003$ & 26.9 & 24 & 0 & 0 & 0 & 0 & $\$ 0$ \\
\hline $12 / 20 / 2003$ & 26.9 & 24 & 0 & 0 & 0 & 0 & $\$ 0$ \\
\hline $12 / 21 / 2003$ & 26.9 & 24 & 0 & 0 & 0 & 0 & $\$ 0$ \\
\hline $12 / 22 / 2003$ & 26.9 & 24 & 0 & 0 & 0 & 0 & $\$ 0$ \\
\hline $12 / 23 / 2003$ & 26.9 & 24 & 0 & 0 & 0 & 0 & $\$ 0$ \\
\hline $12 / 24 / 2003$ & 26.9 & 24 & 0 & 0 & 0 & 0 & $\$ 0$ \\
\hline $12 / 25 / 2003$ & 26.9 & 24 & 0 & 0 & 0 & 0 & $\$ 0$ \\
\hline $12 / 26 / 2003$ & 26.9 & 24 & 0 & 0 & 0 & 0 & $\$ 0$ \\
\hline $12 / 27 / 2003$ & 26.9 & 24 & 0 & 0 & 0 & 0 & $\$ 0$ \\
\hline $12 / 28 / 2003$ & 26.9 & 24 & 0 & 0 & 0 & 0 & $\$ 0$ \\
\hline $12 / 29 / 2003$ & 26.9 & 24 & 0 & 0 & 0 & 0 & $\$ 0$ \\
\hline $12 / 30 / 2003$ & 26.9 & 24 & 0 & 0 & 0 & 0 & $\$ 0$ \\
\hline $12 / 31 / 2003$ & 26.9 & 24 & 0 & 0 & 0 & 0 & $\$ 0$ \\
\hline $1 / 1 / 2004$ & 22.1 & 24 & 0 & 0 & 0 & 0 & $\$ 0$ \\
\hline $1 / 2 / 2004$ & 22.1 & 24 & 0 & 0 & 0 & 0 & $\$ 0$ \\
\hline $1 / 3 / 2004$ & 22.1 & 24 & 0 & 0 & 0 & 0 & $\$ 0$ \\
\hline $1 / 4 / 2004$ & 22.1 & 24 & 0 & 0 & 0 & 0 & $\$ 0$ \\
\hline \multirow[t]{3}{*}{$1 / 5 / 2004$} & 22.1 & 20.0 & 0 & 0 & 0 & 0 & $\$ 0$ \\
\hline & 28.0 & 2.3 & 0.48 & 4.6 & 19.0 & 33 & $\$ 224$ \\
\hline & 28.0 & 1.7 & 0.48 & 3.4 & 14.0 & 24 & $\$ 166$ \\
\hline \multirow[t]{3}{*}{$1 / 6 / 2004$} & 22.1 & 18.8 & 0 & 0 & 0 & 0 & $\$ 0$ \\
\hline & 33.0 & 2.7 & 0.40 & 4.4 & 14.8 & 27 & $\$ 183$ \\
\hline & 31.0 & 2.5 & 0.42 & 4.3 & 16.5 & 29 & $\$ 198$ \\
\hline \multirow[t]{2}{*}{$1 / 7 / 2004$} & 22.1 & 19.5 & 0 & 0 & 0 & 0 & $\$ 0$ \\
\hline & 10.0 & 4.5 & 0.90 & 17.9 & 62.0 & 110 & $\$ 759$ \\
\hline \multirow[t]{3}{*}{$1 / 8 / 2004$} & 22.1 & 18.3 & 0 & 0 & 0 & 0 & $\$ 0$ \\
\hline & 30.0 & 3.6 & 0.41 & 6.1 & 25.8 & 44 & $\$ 303$ \\
\hline & 27.0 & 2.1 & 0.47 & 4.1 & 18.5 & 31 & $\$ 215$ \\
\hline $1 / 9 / 2004$ & 22.1 & 21.3 & 0 & 0 & 0 & 0 & $\$ 0$ \\
\hline
\end{tabular}




\begin{tabular}{|c|c|c|c|c|c|c|c|}
\hline & 27.0 & 1.8 & 0.47 & 3.5 & 15.9 & 27 & $\$ 184$ \\
\hline & 10.0 & 0.9 & 0.46 & 1.8 & 16.4 & 25 & $\$ 173$ \\
\hline \multirow[t]{2}{*}{$1 / 10 / 2004$} & 22.1 & 20.2 & 0 & 0 & 0 & 0 & $\$ 0$ \\
\hline & 22.1 & 3.8 & 0.90 & 14.6 & 43.7 & 80 & $\$ 554$ \\
\hline $1 / 11 / 2004$ & 22.1 & 24 & 0 & 0 & 0 & 0 & $\$ 0$ \\
\hline $1 / 12 / 2004$ & 22.1 & 24 & 0 & 0 & 0 & 0 & $\$ 0$ \\
\hline \multirow{3}{*}{$1 / 13 / 2004$} & 22.1 & 19.3 & 0 & 0 & 0 & 0 & $\$ 0$ \\
\hline & 37.0 & 1.8 & 0.35 & 2.5 & 5.9 & 12 & $\$ 81$ \\
\hline & 32.0 & 2.9 & 0.41 & 4.9 & 17.6 & 31 & $\$ 213$ \\
\hline \multirow[t]{3}{*}{$1 / 14 / 2004$} & 22.1 & 19.9 & 0 & 0 & 0 & 0 & $\$ 0$ \\
\hline & 26.0 & 1.7 & 0.46 & 3.3 & 15.9 & 26 & $\$ 182$ \\
\hline & 35.0 & 2.4 & 0.39 & 3.8 & 10.6 & 20 & $\$ 137$ \\
\hline \multirow[t]{4}{*}{$1 / 15 / 2004$} & 22.1 & 18.7 & 0 & 0 & 0 & 0 & $\$ 0$ \\
\hline & 38.0 & 2.5 & 0.34 & 3.4 & 6.9 & 14 & $\$ 98$ \\
\hline & 28.0 & 1.5 & 0.48 & 3.0 & 12.4 & 21 & $\$ 146$ \\
\hline & 27.0 & 1.3 & 0.34 & 1.8 & 11.5 & 18 & $\$ 126$ \\
\hline $1 / 16 / 2004$ & 22.1 & 24 & 0 & 0 & 0 & 0 & $\$ 0$ \\
\hline \multirow[t]{2}{*}{$1 / 17 / 2004$} & 22.1 & 17.2 & 0 & 0 & 0 & 0 & $\$ 0$ \\
\hline & 16.0 & 6.8 & 0.47 & 13.9 & 101.1 & 158 & $\$ 1,093$ \\
\hline $1 / 18 / 2004$ & 22.1 & 24 & 0 & 0 & 0 & 0 & $\$ 0$ \\
\hline $1 / 19 / 2004$ & 22.1 & 24 & 0 & 0 & 0 & 0 & $\$ 0$ \\
\hline \multirow[t]{3}{*}{$1 / 20 / 2004$} & 22.1 & 20.4 & 0 & 0.0 & 0 & 0 & $\$ 0$ \\
\hline & 19.0 & 1.4 & 0.85 & 5.1 & 18.5 & 33 & $\$ 224$ \\
\hline & 18.0 & 2.2 & 0.85 & 8.1 & 30.3 & 53 & $\$ 364$ \\
\hline \multirow[t]{2}{*}{$1 / 21 / 2004$} & 22.1 & 18.9 & 0 & 0 & 0 & 0 & $\$ 0$ \\
\hline & 18.0 & 5.1 & 0.85 & 18.7 & 70.2 & 122 & $\$ 845$ \\
\hline \multirow[t]{4}{*}{$1 / 22 / 2004$} & 22.1 & 17.6 & 0 & 0 & 0 & 0 & $\$ 0$ \\
\hline & 25.0 & 1.7 & 0.76 & 5.4 & 16.9 & 31 & $\$ 212$ \\
\hline & 25.0 & 1.8 & 0.80 & 6.1 & 17.8 & 33 & $\$ 227$ \\
\hline & 25.0 & 2.9 & 0.44 & 5.4 & 28.8 & 47 & $\$ 324$ \\
\hline \multirow[t]{5}{*}{$1 / 23 / 2004$} & 22.1 & 11.3 & 0 & 0.0 & 0 & 0 & $\$ 0$ \\
\hline & 25.0 & 1.6 & 0.8 & 5.4 & 15.9 & 29 & $\$ 202$ \\
\hline & 12.0 & 2.7 & 0.43 & 5.1 & 46.1 & 71 & $\$ 486$ \\
\hline & 18.0 & 5.9 & 0.44 & 11.2 & 81.2 & 127 & $\$ 878$ \\
\hline & 18.0 & 2.5 & 0.86 & 9.3 & 34.4 & 60 & $\$ 415$ \\
\hline \multirow[t]{3}{*}{$1 / 24 / 2004$} & 22.1 & 16.7 & 0 & 0.0 & 0 & 0 & $\$ 0$ \\
\hline & 21.0 & 5.9 & 0.8 & 20.2 & 71.5 & 126 & $\$ 871$ \\
\hline & 20.0 & 1.4 & 0.8 & 4.8 & 17.7 & 31 & $\$ 214$ \\
\hline \multirow[t]{3}{*}{$1 / 25 / 2004$} & 22.1 & 13.5 & 0 & 0.0 & 0 & 0 & $\$ 0$ \\
\hline & 17.0 & 6.6 & 0.5 & 14.3 & 94.5 & 150 & $\$ 1,034$ \\
\hline & 12.0 & 3.9 & 0.5 & 8.6 & 66.6 & 104 & $\$ 714$ \\
\hline \multirow[t]{3}{*}{$1 / 26 / 2004$} & 22.1 & 19.6 & 0 & 0.0 & 0.0 & 0 & $\$ 0$ \\
\hline & 31.0 & 1.3 & 0.86 & 4.6 & 8.6 & 18 & $\$ 125$ \\
\hline & 27.0 & 1.1 & 0.81 & 3.7 & 9.7 & 18 & $\$ 128$ \\
\hline
\end{tabular}




\begin{tabular}{|c|c|c|c|c|c|c|c|}
\hline & 20.0 & 2.0 & 0.42 & 3.6 & 25.3 & 40 & $\$ 275$ \\
\hline \multirow[t]{5}{*}{$1 / 27 / 2004$} & 22.1 & 16.4 & 0 & 0.0 & 0 & 0 & $\$ 0$ \\
\hline & 32.0 & 1.9 & 0.5 & 3.9 & 11.5 & 21 & $\$ 146$ \\
\hline & 30.0 & 3.1 & 0.47 & 6.0 & 22.2 & 39 & $\$ 268$ \\
\hline & 22.0 & 0.6 & 0.44 & 1.1 & 6.9 & 11 & $\$ 77$ \\
\hline & 29.0 & 2.0 & 0.45 & 3.7 & 15.4 & 26 & $\$ 182$ \\
\hline \multirow[t]{3}{*}{$1 / 28 / 2004$} & 22.1 & 17.4 & 0 & 0.0 & 0 & 0 & $\$ 0$ \\
\hline & 22.0 & 1.5 & 0.44 & 2.8 & 17.4 & 28 & $\$ 192$ \\
\hline & 18.0 & 5.1 & 0.45 & 9.9 & 70.2 & 110 & $\$ 761$ \\
\hline \multirow[t]{4}{*}{$1 / 29 / 2004$} & 22.1 & 14.2 & 0 & 0 & 0 & 0 & $\$ 0$ \\
\hline & 24.0 & 3.1 & 0.7 & 9.2 & 32.4 & 57 & $\$ 395$ \\
\hline & 24.0 & 4.7 & 0.44 & 8.7 & 49.2 & 80 & $\$ 550$ \\
\hline & 24.0 & 2.0 & 0.46 & 3.9 & 20.9 & 34 & $\$ 236$ \\
\hline \multirow[t]{3}{*}{$1 / 30 / 2004$} & 22.1 & 15.6 & 0 & 0 & 0 & 0 & $\$ 0$ \\
\hline & 20.0 & 5.5 & 0.52 & 12.3 & 69.7 & 113 & $\$ 778$ \\
\hline & 15.0 & 2.9 & 0.85 & 10.7 & 44.7 & 76 & $\$ 527$ \\
\hline $1 / 31 / 2004$ & 22.1 & 24 & 0 & 0 & 0 & 0 & $\$ 0$ \\
\hline $2 / 1 / 2004$ & 24.2 & 24 & 0 & 0 & 0 & 0 & $\$ 0$ \\
\hline 2/2/2004 & 24.2 & 24 & 0 & 0 & 0 & 0 & $\$ 0$ \\
\hline $2 / 3 / 2004$ & 24.2 & 24 & 0 & 0 & 0 & 0 & $\$ 0$ \\
\hline $2 / 4 / 2004$ & 24.2 & 24 & 0 & 0 & 0 & 0 & $\$ 0$ \\
\hline $2 / 5 / 2004$ & 24.2 & 24 & 0 & 0 & 0 & 0 & $\$ 0$ \\
\hline $2 / 6 / 2004$ & 24.2 & 24 & 0 & 0 & 0 & 0 & $\$ 0$ \\
\hline \multirow[t]{3}{*}{ 2/7/2004 } & 24.2 & 19.2 & 0 & 0 & 0 & 0 & $\$ 0$ \\
\hline & 33.0 & 3.8 & 0.40 & 6.2 & 20.9 & 37 & $\$ 258$ \\
\hline & 32.0 & 1.0 & 0.41 & 1.7 & 6.1 & 11 & $\$ 74$ \\
\hline $2 / 8 / 2004$ & 24.2 & 24 & 0 & 0 & 0 & 0 & $\$ 0$ \\
\hline 2/9/2004 & 24.2 & 24 & 0 & 0 & 0 & 0 & $\$ 0$ \\
\hline $2 / 10 / 2004$ & 24.2 & 24 & 0 & 0 & 0 & 0 & $\$ 0$ \\
\hline $2 / 11 / 2004$ & 24.2 & 24 & 0 & 0 & 0 & 0 & $\$ 0$ \\
\hline \multirow[t]{2}{*}{$2 / 12 / 2004$} & 24.2 & 22.7 & 0 & 0 & 0 & 0 & $\$ 0$ \\
\hline & 28.0 & 1.3 & 0.45 & 2.4 & 10.7 & 18 & $\$ 125$ \\
\hline $2 / 13 / 2004$ & 24.2 & 24 & 0 & 0 & 0 & 0 & $\$ 0$ \\
\hline $2 / 14 / 2004$ & 24.2 & 24 & 0 & 0 & 0 & 0 & $\$ 0$ \\
\hline $2 / 15 / 2004$ & 24.2 & 24 & 0 & 0 & 0 & 0 & $\$ 0$ \\
\hline $2 / 16 / 2004$ & 24.2 & 24 & 0 & 0 & 0 & 0 & $\$ 0$ \\
\hline $2 / 17 / 2004$ & 24.2 & 24 & 0 & 0 & 0 & 0 & $\$ 0$ \\
\hline $2 / 18 / 2004$ & 24.2 & 24 & 0 & 0 & 0 & 0 & $\$ 0$ \\
\hline $2 / 19 / 2004$ & 24.2 & 24 & 0 & 0 & 0 & 0 & $\$ 0$ \\
\hline
\end{tabular}




\begin{tabular}{|c|c|c|c|c|c|c|c|}
\hline $2 / 20 / 2004$ & 24.2 & 24 & 0 & 0 & 0 & 0 & $\$ 0$ \\
\hline \multirow[t]{4}{*}{$2 / 21 / 2004$} & 24.2 & 19.0 & 0 & 0 & 0 & 0 & $\$ 0$ \\
\hline & 39.0 & 2.0 & 0.20 & 1.6 & 4.4 & 8 & $\$ 57$ \\
\hline & 39.0 & 2.0 & 0.30 & 2.4 & 4.4 & 9 & $\$ 65$ \\
\hline & 39.0 & 1.0 & 0.35 & 1.4 & 2.2 & 5 & $\$ 34$ \\
\hline $2 / 22 / 2004$ & 24.2 & 24 & 0 & 0 & 0 & 0 & $\$ 0$ \\
\hline $2 / 23 / 2004$ & 24.2 & 24 & 0 & 0 & 0 & 0 & $\$ 0$ \\
\hline $2 / 24 / 2004$ & 24.2 & 24 & 0 & 0 & 0 & 0 & $\$ 0$ \\
\hline $2 / 25 / 2004$ & 24.2 & 24 & 0 & 0 & 0 & 0 & $\$ 0$ \\
\hline $2 / 26 / 2004$ & 24.2 & 24 & 0 & 0 & 0 & 0 & $\$ 0$ \\
\hline $2 / 27 / 2004$ & 24.2 & 24 & 0 & 0 & 0 & 0 & $\$ 0$ \\
\hline $2 / 28 / 2004$ & 24.2 & 24 & 0 & 0 & 0 & 0 & $\$ 0$ \\
\hline $2 / 29 / 2004$ & 24.2 & 24 & 0 & 0 & 0 & 0 & $\$ 0$ \\
\hline $3 / 1 / 2004$ & 31.8 & 24 & 0 & 0 & 0 & 0 & $\$ 0$ \\
\hline $3 / 2 / 2004$ & 31.8 & 24 & 0 & 0 & 0 & 0 & $\$ 0$ \\
\hline $3 / 3 / 2004$ & 31.8 & 24 & 0 & 0 & 0 & 0 & $\$ 0$ \\
\hline $3 / 4 / 2004$ & 31.8 & 24 & 0 & 0 & 0 & 0 & $\$ 0$ \\
\hline $3 / 5 / 2004$ & 31.8 & 24 & 0 & 0 & 0 & 0 & $\$ 0$ \\
\hline $3 / 6 / 2004$ & 31.8 & 24 & 0 & 0 & 0 & 0 & $\$ 0$ \\
\hline $3 / 7 / 2004$ & 31.8 & 24 & 0 & 0 & 0 & 0 & $\$ 0$ \\
\hline \multirow[t]{5}{*}{$3 / 8 / 2004$} & 31.8 & 14.9 & 0 & 0 & 0 & 0 & $\$ 0$ \\
\hline & 37.0 & 2.7 & 0.35 & 3.8 & 8.9 & 18 & $\$ 121$ \\
\hline & 34.0 & 2.0 & 0.37 & 3.0 & 9.9 & 18 & $\$ 123$ \\
\hline & 34.0 & 1.5 & 0.37 & 2.3 & 7.4 & 13 & $\$ 92$ \\
\hline & 32.0 & 2.9 & 0.39 & 4.7 & 17.6 & 31 & $\$ 211$ \\
\hline $3 / 9 / 2004$ & 31.8 & 24 & 0 & 0 & 0 & 0 & $\$ 0$ \\
\hline $3 / 10 / 2004$ & 31.8 & 24 & 0 & 0 & 0 & 0 & $\$ 0$ \\
\hline $3 / 11 / 2004$ & 31.8 & 24 & 0 & 0 & 0 & 0 & $\$ 0$ \\
\hline \multirow[t]{2}{*}{$3 / 12 / 2004$} & 31.8 & 22.3 & 0 & 0 & 0 & 0 & $\$ 0$ \\
\hline & 25.0 & 1.7 & 0.43 & 3.1 & 16.9 & 27 & $\$ 189$ \\
\hline $3 / 13 / 2004$ & 31.8 & 24 & 0 & 0 & 0 & 0 & $\$ 0$ \\
\hline $3 / 14 / 2004$ & 31.8 & 24 & 0 & 0 & 0 & 0 & $\$ 0$ \\
\hline $3 / 15 / 2004$ & 31.8 & 24 & 0 & 0 & 0 & 0 & $\$ 0$ \\
\hline $3 / 16 / 2004$ & 31.8 & 24 & 0 & 0 & 0 & 0 & $\$ 0$ \\
\hline $3 / 17 / 2004$ & 31.8 & 24 & 0 & 0 & 0 & 0 & $\$ 0$ \\
\hline $3 / 18 / 2004$ & 31.8 & 24 & 0 & 0 & 0 & 0 & $\$ 0$ \\
\hline $3 / 19 / 2004$ & 31.8 & 24 & 0 & 0 & 0 & 0 & $\$ 0$ \\
\hline $3 / 20 / 2004$ & 31.8 & 24 & 0 & 0 & 0 & 0 & $\$ 0$ \\
\hline $3 / 21 / 2004$ & 31.8 & 24 & 0 & 0 & 0 & 0 & $\$ 0$ \\
\hline
\end{tabular}




\begin{tabular}{|c|c|c|c|c|c|c|c|}
\hline $3 / 22 / 2004$ & 31.8 & 20.8 & 0 & 0 & 0 & 0 & $\$ 0$ \\
\hline & 30.0 & 3.2 & 0.40 & 5.3 & 22.9 & 39 & $\$ 268$ \\
\hline $3 / 23 / 2004$ & 31.8 & 24 & 0 & 0 & 0 & 0 & $\$ 0$ \\
\hline $3 / 24 / 2004$ & 31.8 & 24 & 0 & 0 & 0 & 0 & $\$ 0$ \\
\hline $3 / 25 / 2004$ & 31.8 & 24 & 0 & 0 & 0 & 0 & $\$ 0$ \\
\hline $3 / 26 / 2004$ & 31.8 & 24 & 0 & 0 & 0 & 0 & $\$ 0$ \\
\hline $3 / 27 / 2004$ & 31.8 & 24 & 0 & 0 & 0 & 0 & $\$ 0$ \\
\hline $3 / 28 / 2004$ & 31.8 & 24 & 0 & 0 & 0 & 0 & $\$ 0$ \\
\hline $3 / 29 / 2004$ & 31.8 & 24 & 0 & 0 & 0 & 0 & $\$ 0$ \\
\hline $3 / 30 / 2004$ & 31.8 & 24 & 0 & 0 & 0 & 0 & $\$ 0$ \\
\hline $3 / 31 / 2004$ & 31.8 & 24 & 0 & 0 & 0 & 0 & $\$ 0$ \\
\hline
\end{tabular}

From the analysis, it was calculated that the total heat produced from natural gas burner is $2800 \mathrm{MBtu}$. The cost of natural gas is about $\$ 19,400$ assuming a constant price

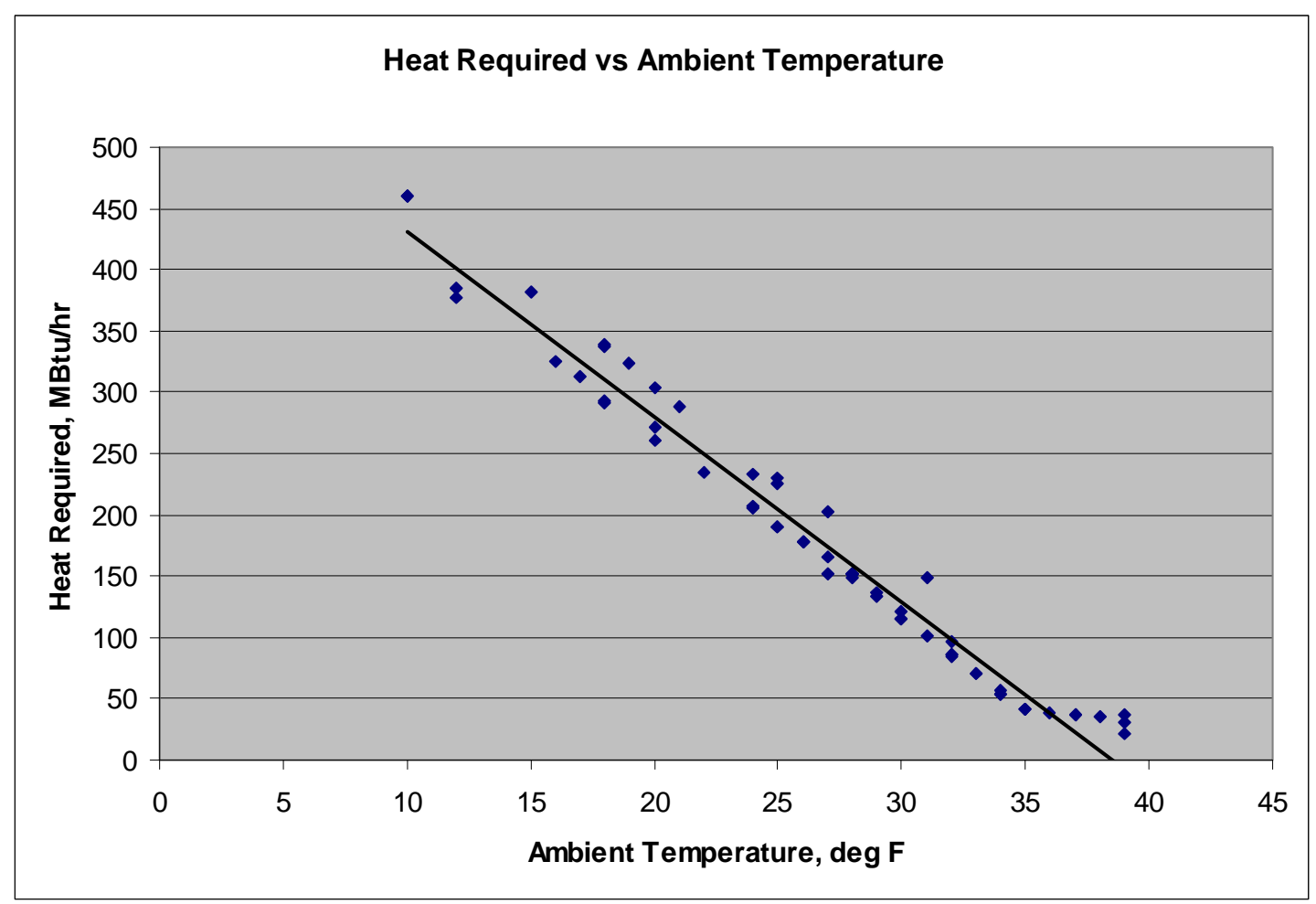

Figure 4.4: Graph of Increased Heat Requirement with Decrease in Temperature of $\$ 5 / \mathrm{MCF}$. An additional $20 \%$ of fuel shall be consumed for start up of the heating system. The historical utility cost of natural gas for M-PRT is mentioned in Appendix E. The heat required for maintaining an ice-free guideway increases with decrease in ambient temperature as shown in Figure 4.4. 


\subsection{Emissions from Existing Heating System}

Emissions from the natural gas burner are assumed to be $120 \mathrm{lbs}$ per MBtu of heat produced. The cost of equipment to reduce emissions through the use of Selective Catalytic Reduction (SCR) is 64 mills per kW-hr of heat produced or $\$ 18.75 / \mathrm{MBtu}$ [21]. The emissions from an operating existing heating system (existing heating system is operated only when it snows) also increase with the decrease in ambient temperature as

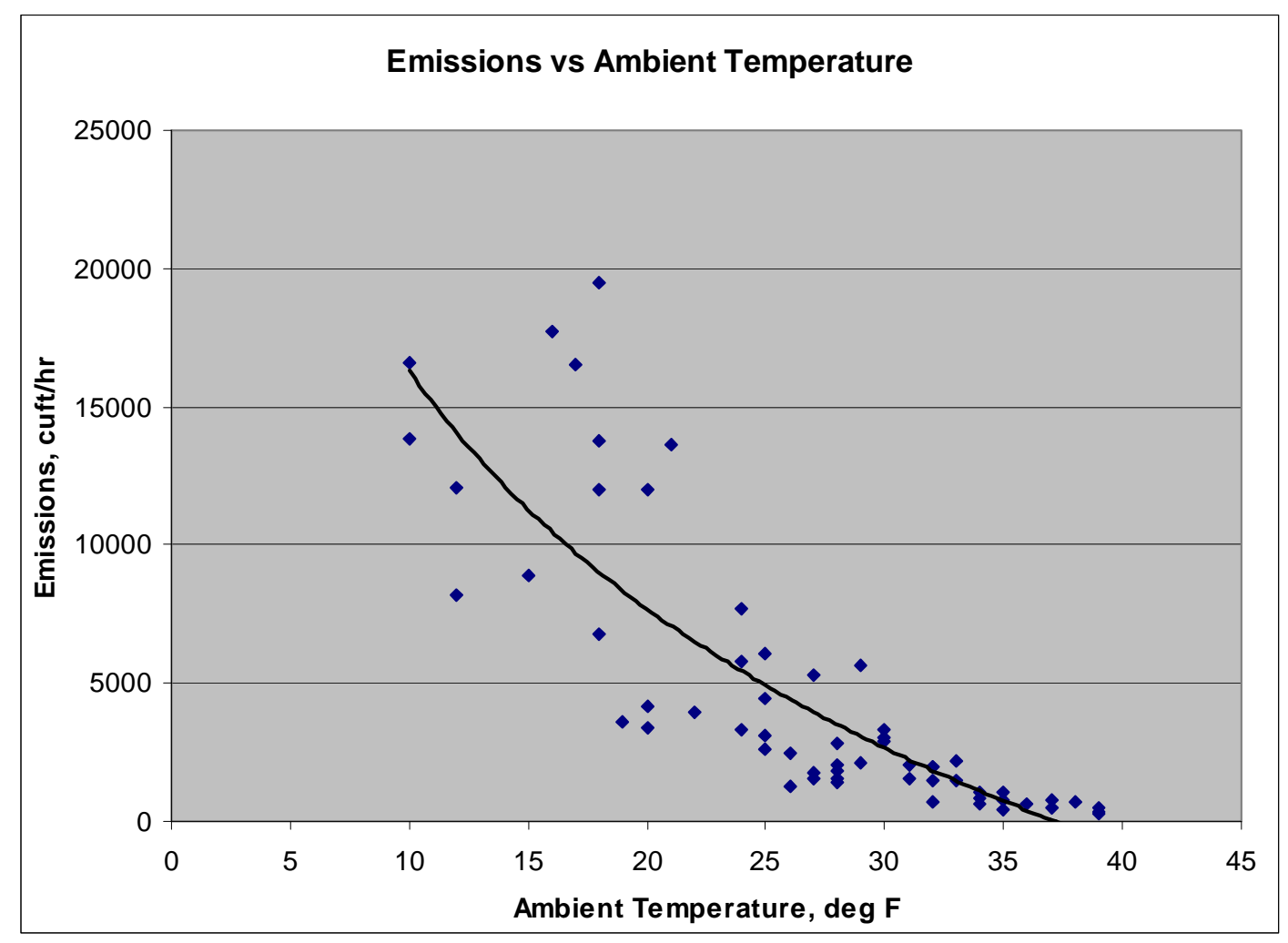

Figure 4.5: NOx Emissions from Existing Heating System

shown in Figure 4.5. The undesired gases that are released through emissions were the oxides of nitrogen (NOx). These emissions increase the net cost of operating the system, as shown by Equation 4.17.

Cost for existing heating system $=($ Fuel used per hour in cu.ft $)(\operatorname{cost}$ of fuel used per hour $)$

$$
+\frac{O \& M \text { cost }}{\text { hours of operation }}+\frac{\text { Cost due to emissions }}{\text { hours of operation }}
$$




\section{Chapter 5}

\section{Design of Hybrid Guideway Heating System}

\subsection{Introduction}

The Phase 2 of the FTA project was aimed in modernizing the existing equipment and systems in M-PRT and thereby

- Reduce utility and operational costs

- Reduce energy consumption

- Ensure high system reliability

To contribute to the FTA project, a hybrid heating system which uses a fuel cell was designed to reduce the utility and operational cost incurred with the existing guideway heating system of the M-PRT in extremely cold and inclimate weather. In the proposed hybrid heating system, the natural gas burner supplies heat to the guideway only during the snowing conditions. The fuel cell will operate throughout the year to keep the guideway at $43^{\circ} \mathrm{F}$.

\subsection{Characteristics of Hybrid Guideway Heating System}

The hybrid heating system shall be an efficient method of producing heat for the guideway in the M-PRT system. As the fuel cell heater can be capable of producing heat at a constant rate with low emissions and economically, it is expected to provide a major part of heat to the guideway. The remaining heat is supplied by natural gas burner making the entire system more cost effective.

Since the guideway is maintained at a constant temperature of $43^{\circ} \mathrm{F}$ throughout the winter, it will reduce any possible damages that occur due to varying temperatures. The other advantages include

- Improved system reliability

- Extended life of the guideway

- Lower start-up costs for gas burner 


\subsection{Incorporation of Fuel Cell as a Source of Heat to Hybrid Heating System}

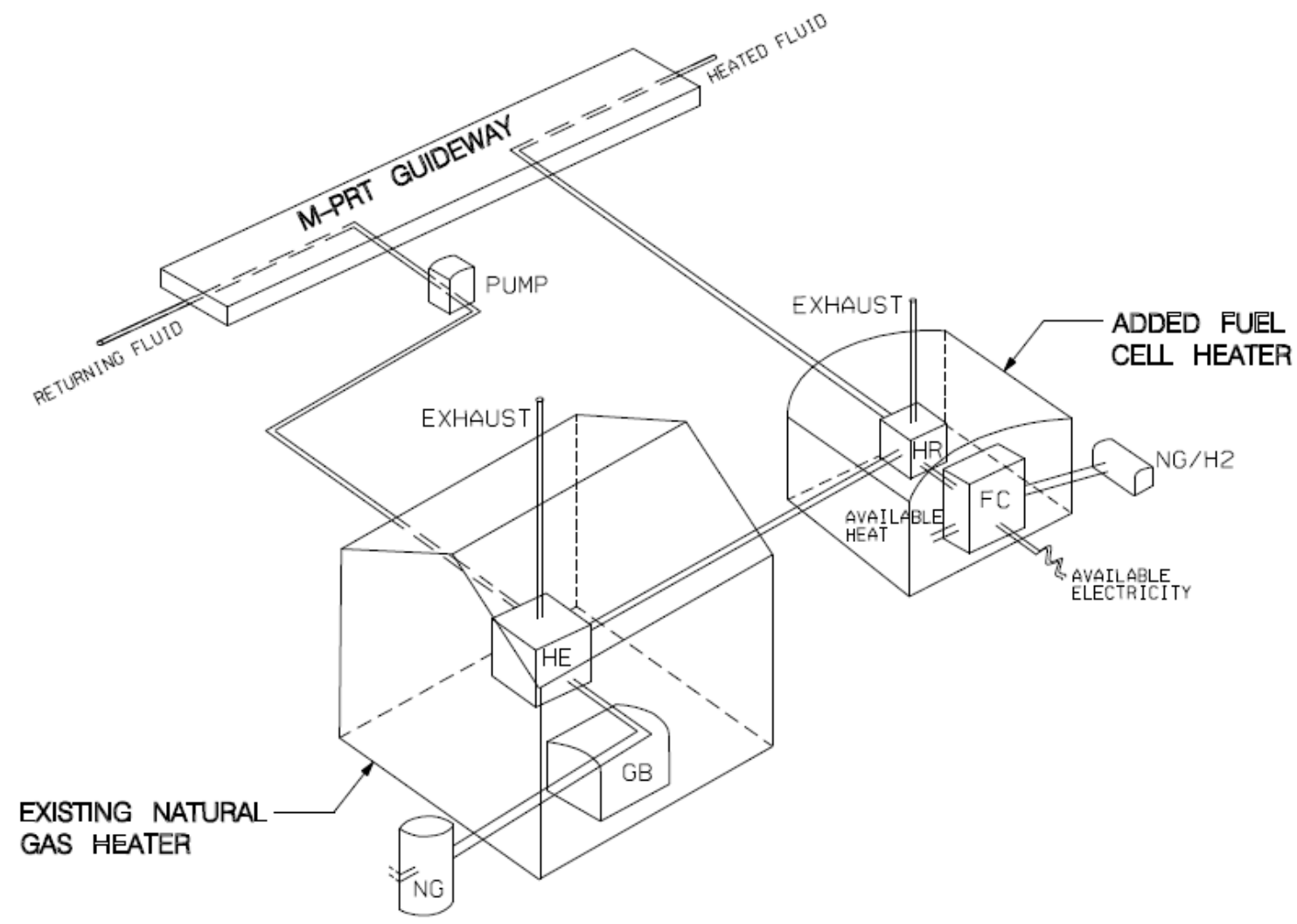

where GB - Gas Burner; FC - Fuel Cell; HR - Heat Recouperator; HE - Heat Exchanger; NG - Natural Gas; H2 - Hydrogen;

Figure 5.1: Illustrative Diagram for Hybrid Guideway Heating System

Fuel cell is being incorporated in the hybrid heating system. High heat is released from the fuel cell in the form of steam. This heat energy is transferred to the guideway through a heat recouperator. The use of hydrogen as another fuel to fuel cell shall provide the flexibility of using an alternate fuel in hybrid heating system. Fuel cell can be an economic way for producing heat energy. The Figure 5.1 shows the illustrative diagram of the proposed hybrid heating system where the fuel cell heater is added to the existing natural gas burner. The returning fluid will flow through the heat exchanger and heat recouperator. The Figure 5.2 is a schematic that shows different locations on the hybrid guideway heating system including existing gas burner and fuel cell as sources of heat. The numbers 1 to 8 in the schematic represents different points in the hybrid 
guideway heating system where there can be a temperature or pressure variation as detailed in section 5.7 .

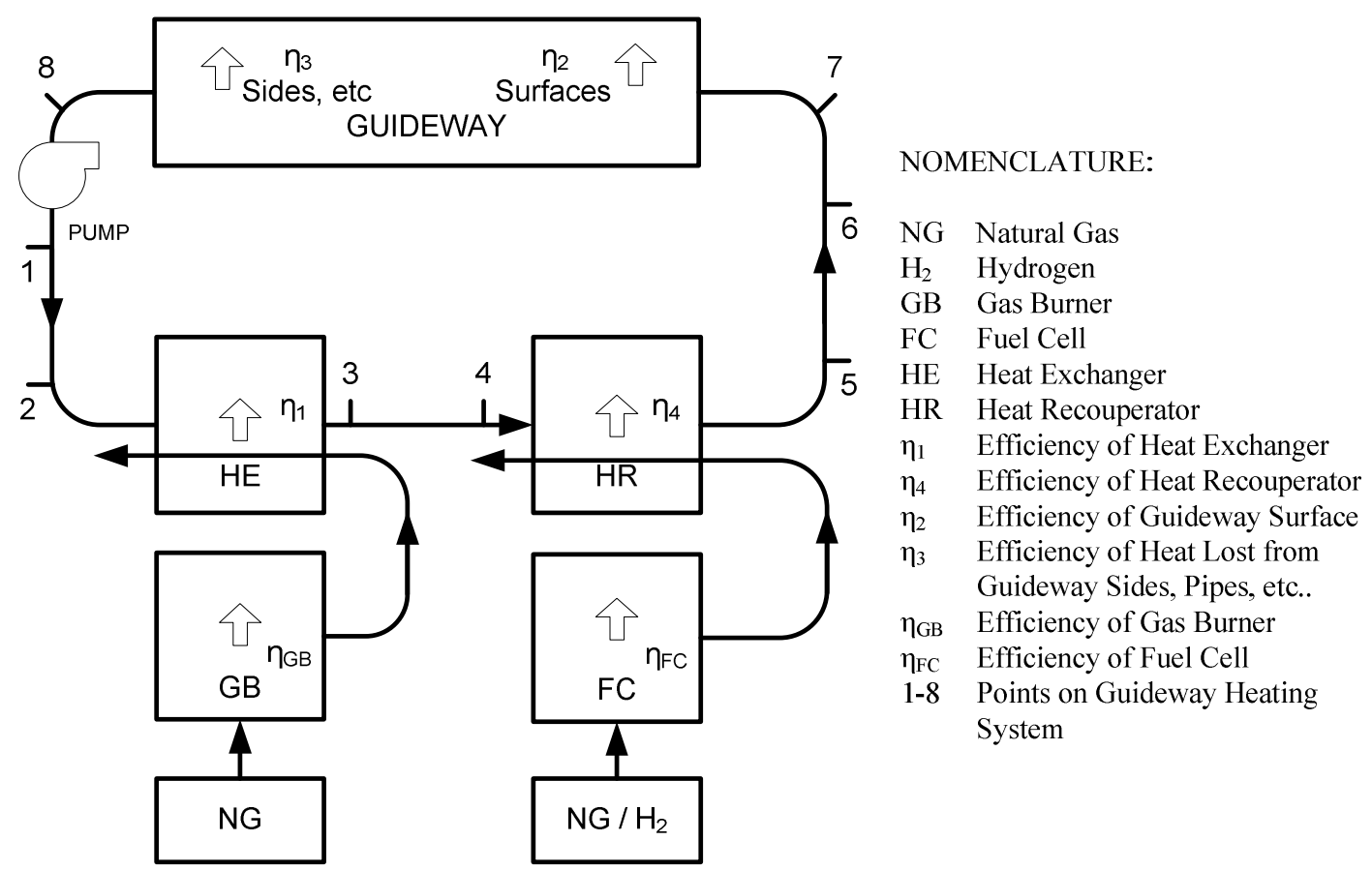

Figure 5.2: Schematic of Hybrid Guideway Heating System

A block diagram of the hybrid heating system is shown in Figure 5.3. A fuel cell (FC) with heat recouperator is added to the existing heating system. The controllers are connected to the inlet fuel supply of natural gas heater and at the outlet of fuel cell to control the fuel flow and heat supplied to the guideway. The controllers detect the ambient and guideway temperatures and control the supply of heat to the guideway. Since fuel cell is operated throughout the year supplying constant heat, the hydrogen gas supply into the fuel cell remains constant. Fuel Cell supplies the required heat to the guideway and the additional heat is used for commercial purposes. Fuel Cell also generates electrical energy at an efficiency of $40 \%$. 


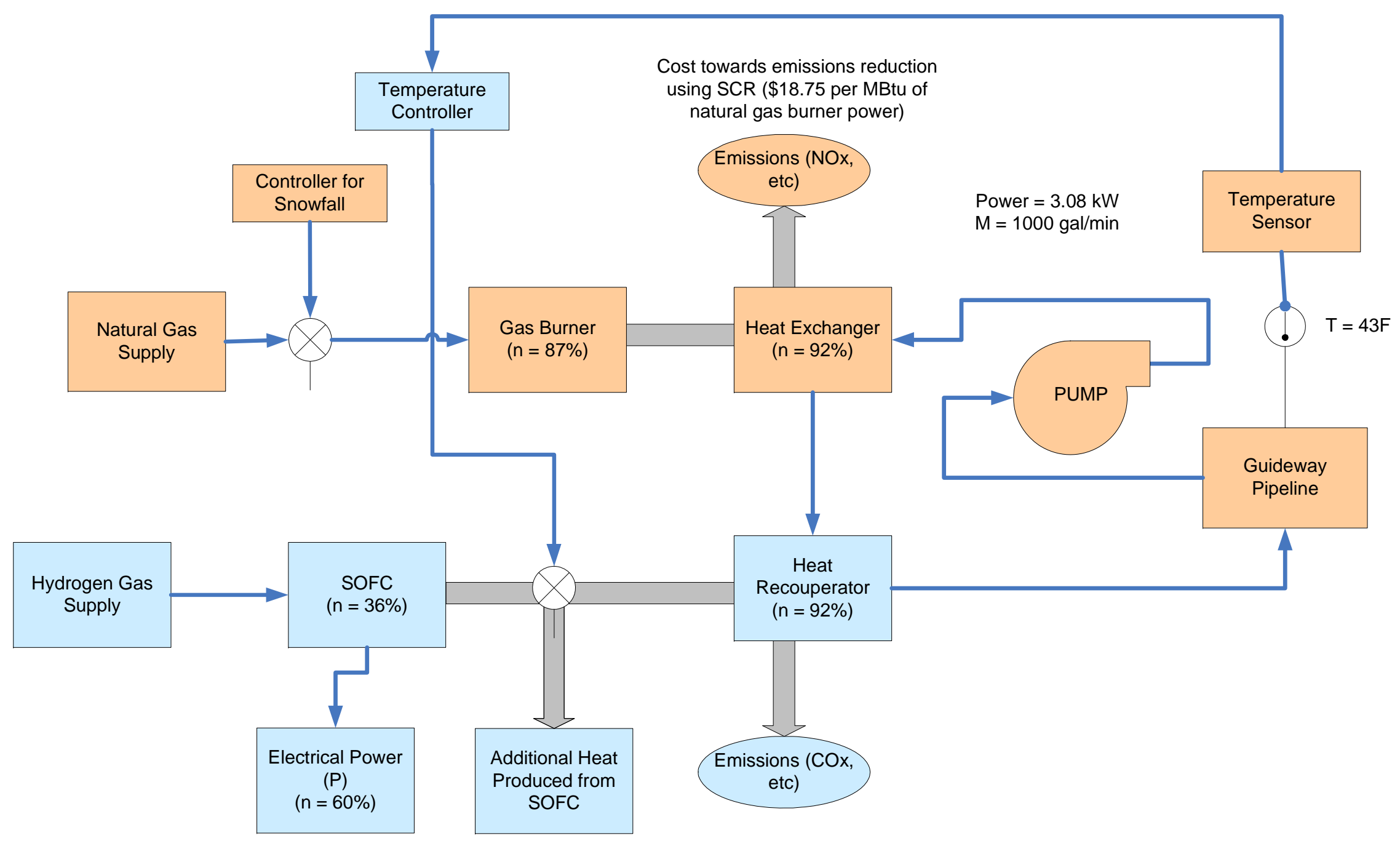

Figure 5.3: Block Diagram of Hybrid Heating System 


\subsection{Fuel Cell as a Source of Heat}

Among all the potential alternate sources of heat, fuel cell (SOFC) has a got more prominence as it is a high temperature fuel cell.

Fuel cell is a complete solid-state device that uses an oxide ion-conducting ceramic as the electrolyte. In fuel cell, the negatively charged oxygen ion is transferred from the cathode through the electrolyte to the anode. The product water is formed at the anode. The water obtained is in the form of steam, which carries a lot of heat.

\subsubsection{Specifications of Fuel Cell}

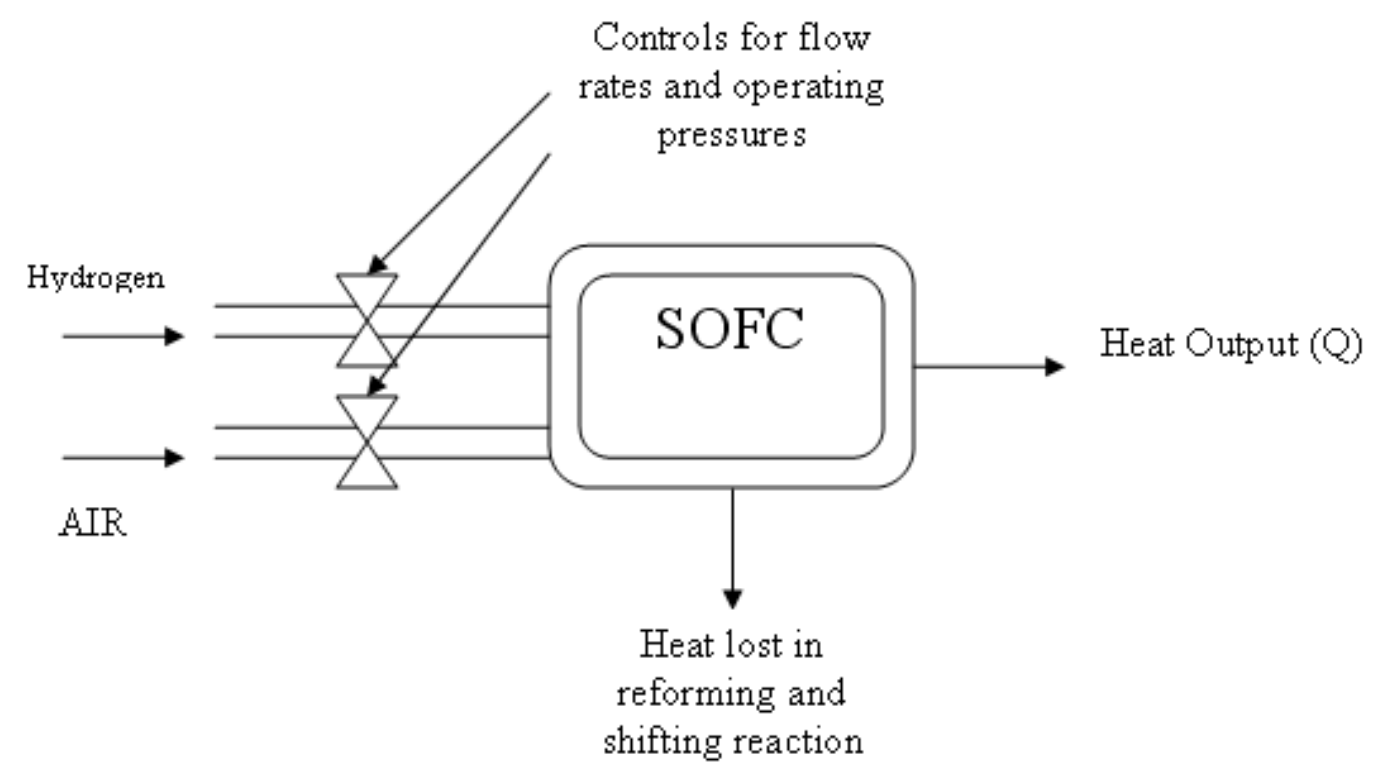

Figure 5.4: Heat Available from SOFC

The fuel cell used in the hybrid heating system is assumed to have the specifications shown in Table 5.1.

Table 5.1: Specifications of Fuel Cell Used

\begin{tabular}{|l|l|}
\hline Maximum Operating Temperature of Fuel Cell & $1850^{\circ} \mathrm{F}$ \\
\hline Hydrogen input to Fuel Cell & $3.55 \mathrm{ft}^{3} / \mathrm{sec}$ \\
\hline Inlet Pressure of Hydrogen Gas & $115 \mathrm{psi}$ \\
\hline Operating Thermal Efficiency of Fuel Cell & $36 \%$ \\
\hline
\end{tabular}




\subsubsection{Heat Available for Transfer from Fuel Cell}

The total heat available from the fuel cell is the difference between heat released from the electrochemical reaction and that absorbed in the reforming and shifting reactions. From Figure 5.4, heat output from fuel cell can be determined as

$Q=$ Qreaction - Qreforming - Qshifting

\subsection{Design of Heat Exchanger}

The heat exchanger transfers the heat from the natural gas burner exhaust to the guideway heating fluid. The temperature of the guideway heating fluid needs to increase from $135^{\circ} \mathrm{F}$ to $145^{\circ} \mathrm{F}$.

Assuming the volume flow rate of heated air coming out of gas burner $\left(\mathrm{m}_{\mathrm{h}}\right)$

$$
=14000 \mathrm{ft}^{3} \text { per hour }
$$

Specific heat $\left(\mathrm{C}_{\mathrm{h}}\right)$ of air $=4.1 \mathrm{Btu} / \mathrm{lb} .^{\circ} \mathrm{F}$

Density of air $\left(D_{h}\right)$ at $300 \mathrm{~F}$ is $1.3 \mathrm{lb} / \mathrm{ft}^{3}$

Volume flow rate of the heating fluid (50\% ambitrol NTC and 50\% water) $\left(\mathrm{m}_{\mathrm{c}}\right)$

$$
\begin{aligned}
& =1000 \text { gallons per min } \\
& =7800 \mathrm{ft}^{3} \text { per hour }
\end{aligned}
$$

Density of ambitrol $\left(D_{c}\right)$ at $100 \mathrm{~F}$ is $64.42 \mathrm{lb} / \mathrm{ft}^{3}$

Specific heat $\left(\mathrm{C}_{\mathrm{c}}\right)$ of the heating fluid (50\% ambitrol NTC and $50 \%$ water)

$$
=0.9 \mathrm{Btu} / \mathrm{lb} \cdot{ }^{\circ} \mathrm{F}
$$

Assuming ideal conditions in the heat exchanger, the heat lost by hot fluid (dQ) is equal to the heat gained my cold fluid (dQ). Using the equation 5.2 below, the outlet temperature of the hot fluid can be calculated.

$$
\begin{aligned}
\mathrm{dQ}=\left(\mathrm{m}_{\mathrm{h}}\right)\left(\mathrm{C}_{\mathrm{h}}\right)\left(\mathrm{D}_{\mathrm{h}}\right)\left(\mathrm{T}_{\mathrm{h}, \mathrm{L}}-\mathrm{T}_{\mathrm{h}, 0}\right)=\left(\mathrm{m}_{\mathrm{c}}\right)\left(\mathrm{C}_{\mathrm{c}}\right)\left(\mathrm{D}_{\mathrm{h}}\right)\left(\mathrm{T}_{\mathrm{c}, \mathrm{L}}-\mathrm{T}_{\mathrm{c}, 0}\right) \\
=4.5 \mathrm{MBtu} / \mathrm{hr}
\end{aligned}
$$

Temperatures of the inlet and outlet flows are shown in Figure 5.5. Hot exhaust gases are from the natural gas burner and cold fluid is the heating fluid that is pumped through the guideway pipes.

Overall heat transfer co-efficient $(\mathrm{U})=112.2 \mathrm{Btu} / \mathrm{ft}^{2} \cdot{ }^{2} \mathrm{hr} \cdot{ }^{\circ} \mathrm{F}$

Perimeter of the outer diameter of heat exchanger $(\mathrm{P})=\pi \mathrm{D}$ 
where $\mathrm{D}$ is the outer diameter of heat exchanger

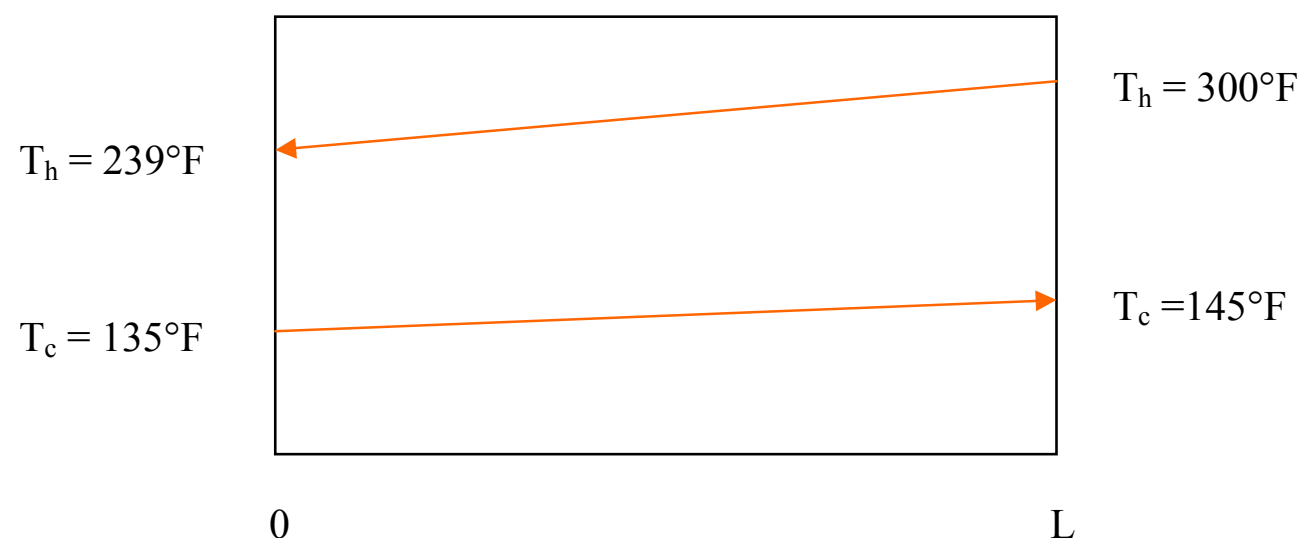

Figure 5.5: Temperature Change in Inlet and Outlet Flows due to Heat Exchanger [13] Logarithmic mean temperature difference $\left(\Delta \mathrm{T}_{\mathrm{m}}\right)$ of heat exchanger is computed as 147.7 using Equation 5.3,

$$
\Delta T_{m}=\frac{\left(T_{h}-T_{c}\right)_{L}-\left(T_{h}-T_{c}\right)_{0}}{\ln \left(\left(T_{h}-T_{c}\right)_{L} /\left(T_{h}-T_{c}\right)_{0}\right)}=147.7
$$

where $T_{h}$ and $T_{c}$ are temperatures of hot and cold fluids respectively at inlet (0) and outlet (L) locations.

Overall heat transfer coefficient $(U)$ is calculated using equation 5.4 below [15]

$$
\frac{1}{U}=\frac{1}{h_{o}}+\frac{x}{k}+\frac{1}{h_{i}}
$$

where

$U$ - Overall heat transfer coefficient (in Btu/ $/ \mathrm{ft}^{2} . \mathrm{hr} .{ }^{\circ} \mathrm{F}$ )

$h_{o}$ - Heat transfer co-efficient of outside hot fluid (air) is $445 \mathrm{Btu} / \mathrm{ft}^{2} . \mathrm{hr}^{\circ}{ }^{\circ} \mathrm{F}$

$h_{i}-$ Heat transfer co-efficient of inner cold fluid (ambitrol) is $972 \mathrm{Btu} / \mathrm{ft}^{2} . \mathrm{hr}^{\circ}{ }^{\circ} \mathrm{F}$

$k$ - Thermal conductivity of tube material (steel) is $26.6 \mathrm{Btu} / \mathrm{ft} . \mathrm{hr} .^{\circ} \mathrm{F}$

$\mathrm{x}-$ Thickness of the tube carrying the heating liquid $(0.15 \mathrm{ft})$

Length of the heat exchanger can be calculated using equation 5.5 below [15]

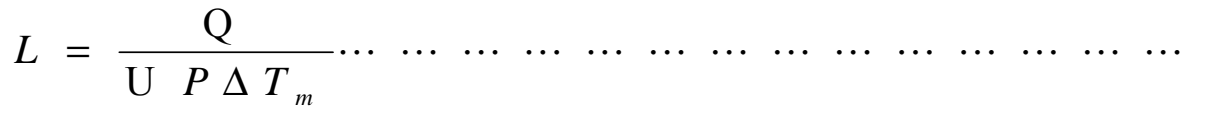

where

$\mathrm{Q}$ - Total heat transfer in the exchanger 
$\mathrm{U}$ - Overall heat transfer coefficient

$P$ - Perimeter of the cold fluid pipe

Hence, if the diameter of the heat exchanger is 7 feet, then the length of the heat exchanger is computed to be 2 feet. Increasing the perimeter of the heat exchanger decreases its length.

\subsection{Design of Heat Recouperator}

A recouperator that transfers heat from the fuel cell to the guideway heating fluid is being designed. It is a counter flow co-axial heat exchanger.

Assuming the volume flow rate of heated gases coming out of fuel cell $\left(\mathrm{m}_{\mathrm{h}}\right)=3000 \mathrm{ft}^{3}$ per hour

Specific heat $\left(\mathrm{C}_{\mathrm{h}}\right)$ of air at $200^{\circ} \mathrm{F}=3.48 \mathrm{Btu} / \mathrm{lb} .^{\circ} \mathrm{F}$

Density of air $\left(\mathrm{D}_{\mathrm{h}}\right)$ at $200^{\circ} \mathrm{F}=0.95 \mathrm{lb} / \mathrm{ft}^{3}$

Volume flow rate of heating fluid $(50 \%$ ambitrol NTC $)=7800 \mathrm{ft}^{3}$ per hour

Density of ambitrol $\left(D_{c}\right)$ is $64.42 \mathrm{lb} / \mathrm{ft}^{3}$

Specific heat $(\mathrm{Cp})$ of heating fluid $(50 \%$ ambitrol NTC $)=0.9 \mathrm{Btu} / \mathrm{lb} .{ }^{\circ} \mathrm{F}$

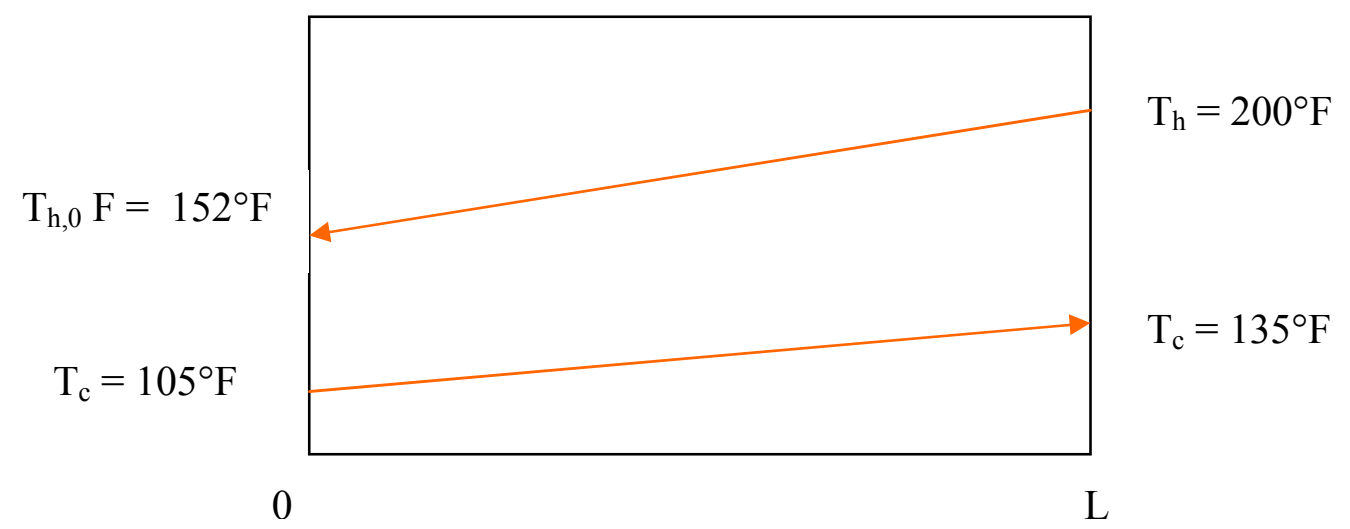

Figure 5.6: Temperature Changes in Inlet and Outlet Flows through Heat Recouperator [13]

In Figure 5.6, the hot gas is the exhaust from the fuel cell and the cold fluid is the heating fluid for the guideway.

Logarithmic mean temperature difference $(\Delta \mathrm{Tm})$ is observed to be $56^{\circ} \mathrm{F}$.

Length of the heat exchanger

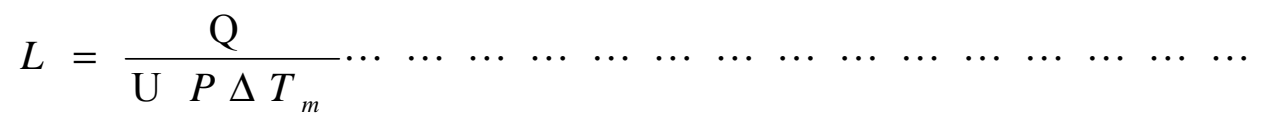


The Overall heat transfer co-efficient is $15.9 \mathrm{Btu} / \mathrm{ft} 2 . \mathrm{hr} .^{\circ} \mathrm{F}$

The length of heat recouperator is computed to be 11 feet and the diameter to be 7 feet.

\subsection{Temperature and Pressure Variations along Guideway Heating System}

The heating fluid remains a liquid throughout the cycle. The fluid is heated to a maximum temperature of $145^{\circ} \mathrm{F}$. This avoids any possibility of changing its state from liquid to vapor. The boiling point of the $50 \%$ ambitrol NTC is $221^{\circ} \mathrm{F}$ and freezing point

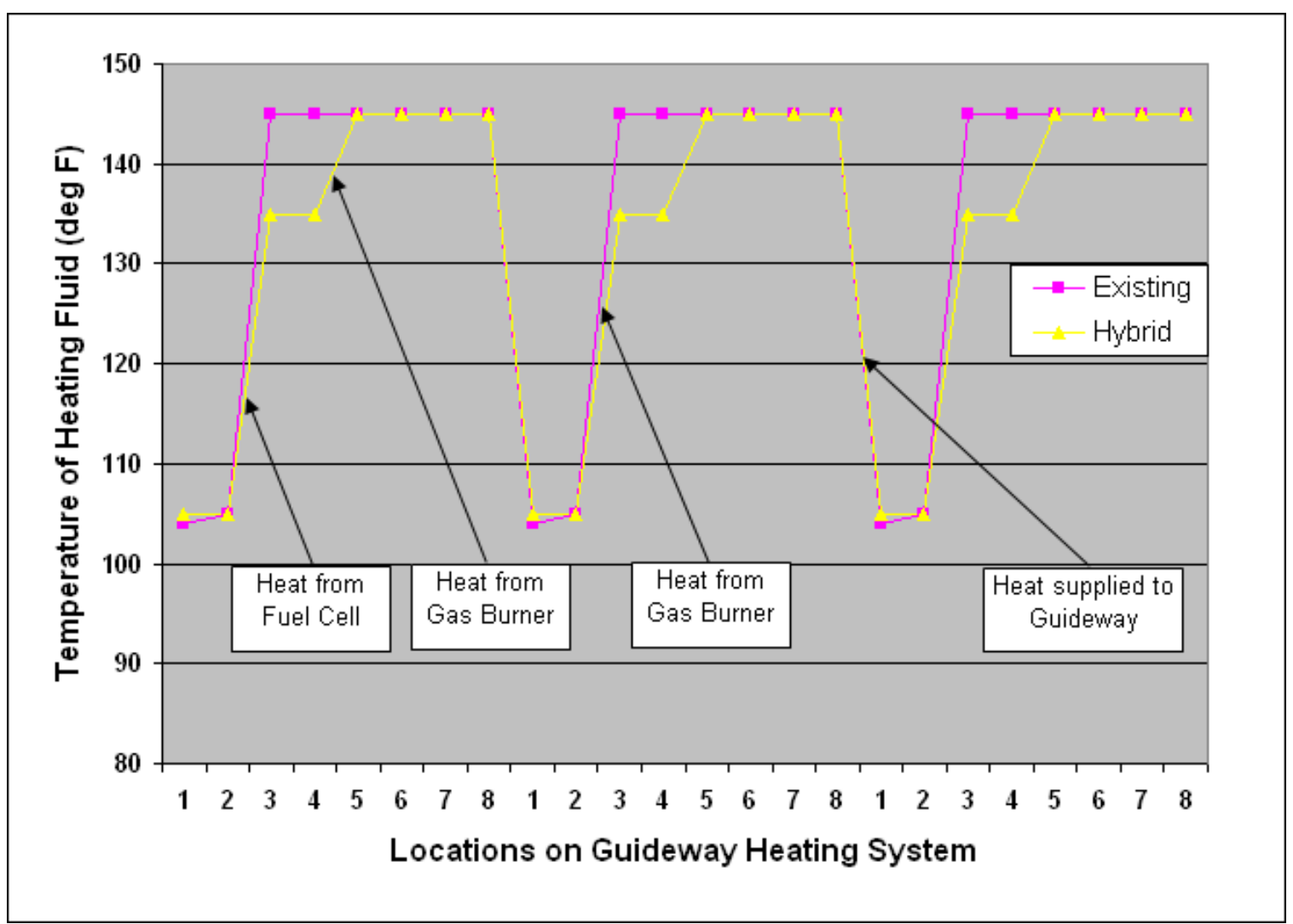

Figure 5.7: Temperature Variation along Guideway Heating System

is $-23^{\circ} \mathrm{F}$. The flow rate remains constant throughout the guideway system. Figure 5.7 shows the variation of temperature along the guideway heating system at different locations (mentioned in Figure 5.2). The heat from fuel cell increases the temperature of the fluid from $105^{\circ} \mathrm{F}$ to $135^{\circ} \mathrm{F}$. The heat from gas burner further increases its temperature from $135^{\circ} \mathrm{F}$ to $145^{\circ} \mathrm{F}$ when it snows.

The pressure variation is represented in Figure 5.8 at different locations on the guideway (as mentioned in Figure 5.1). For the heating fluid, a 50\% ambitrol NTC, there 
is a pressure drop of 0.8 psi per $100 \mathrm{ft}$ of pipe for an 8 -inch diameter pipe and 3 psi per $100 \mathrm{ft}$ of pipe for a 6-inch diameter pipe. The pressure variation is lesser between locations 1 and 7, but a sharp fall between points 7 and 8. The area between locations 7 and 8 represents the guideway of the M-PRT which is approximately $3000 \mathrm{ft}$ in length. Hence, a pressure drop is observed at the end of the guideway.

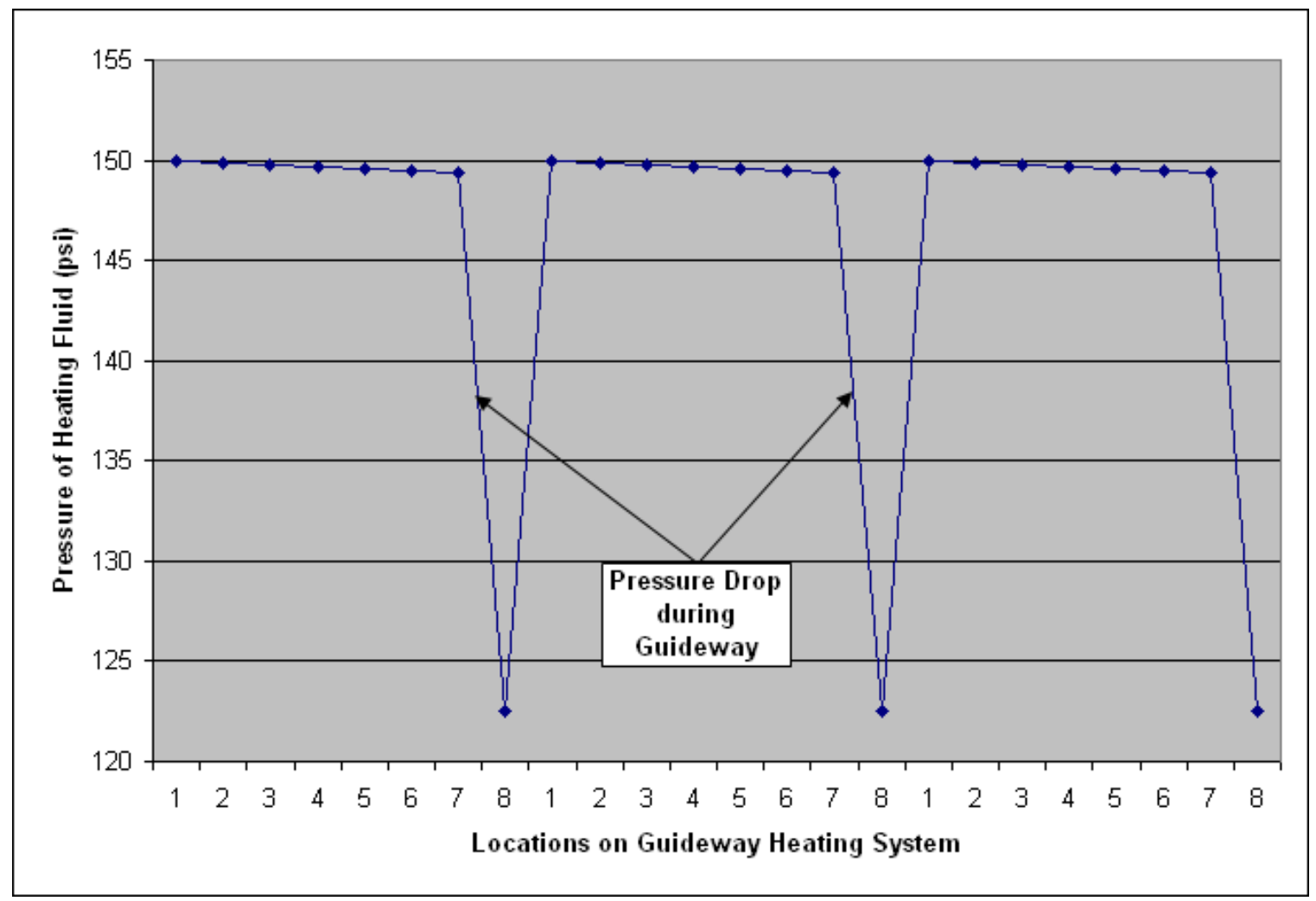

Figure 5.8: Pressure Variation along Guideway Heating System 


\section{Chapter 6}

\section{Analysis of Hybrid Guideway Heating System}

\subsection{Heat Supplied through Hybrid Guideway Heating System}

The heat required to melt snow depends on four atmospheric factors: (1) rate of snowfall, (2) air temperature, (3) relative humidity and (4) wind speed.

The snow melting system must first melt the snow and then evaporate the resulting water film. The rate of snowfall is a determining factor for calculating the heat required to melt the snow. The evaporation rate of the melted snow from the guideway is affected by the wind speed and by the difference in vapor pressure between the air and the melted snow. Since the vapor pressure is determined by the relative humidity and temperature of the air, the resulting evaporation rate varies with changes in air temperature, relative humidity and wind speed.

The fuel cell is used as the primary source of heat. The heat generated by fuel cell is high in temperature and the exhaust gases that carry the heat have minimum undesired pollutants. Therefore, the hybrid heating system is designed in such a way that the valves controlling the heat supply are dependant on the ambient temperature.

\subsection{Detailed Description of Hybrid Guideway Heating System}

The hybrid guideway heating system includes a fuel cell and heat recouperator in addition to the existing heating system.

The data for the guideway fluid, natural gas heater and fuel cell heater are described in subsections below.

\subsubsection{Guideway Fluid}

Fluid in section 3 of guideway is pumped through $2950 \mathrm{ft}$ of guideway pipe at a rate of $1000 \mathrm{gal} / \mathrm{min}$. 


\subsubsection{Natural Gas Heater}

Cost of natural gas is $\$ 5$ per MBtu, as per AEO 2007 projections, as shown in Figure 3.3. Natural gas heater consists of natural gas burner (combustion efficiency $=87 \%$ ) and heat exchanger (efficiency $=92 \%$ ) and thus has an operating efficiency of 80 percent. Natural gas heater in existing heating system is able to heat the guideway fluid to $145^{\circ} \mathrm{F}$ as the fluid exits the heat exchanger to the main guideway pipe. The returning fluid from the main guideway pipe enters the heater at $105^{\circ} \mathrm{F}$. Heater is operated on an average for $6 \%$ of winter and $2 \%$ in a year. Heat capacity of Natural gas is $1000 \mathrm{Btu} / \mathrm{ft}^{3}$ [9].

\subsubsection{Fuel Cell Heater}

Fuel cell heater is used to heat the guideway during the winter to keep guideway at $43^{\circ} \mathrm{F}$. Fuel cell has a startup inlet fuel pressure of $115 \mathrm{psi}$ and produces heated exhaust at $1850^{\circ} \mathrm{F}$. Fuel cell produces electricity at $40 \%$ efficiency, which generates electrical savings. Fuel cell has a heat generation efficiency of $36 \%$ and heat recouperator efficiency of $92 \%$ and thus has an effective thermal operating efficiency of $33 \%$.

\subsubsection{Derivation of Heat Output from Hybrid Guideway Heating system}

Hybrid heating system uses gas burner and fuel cell to supply heat to the guideway. Efficiency of heat exchanger $\left(\eta_{1}\right)$ and heat recouperator $\left(\eta_{4}\right)$ in hybrid heating system are both assumed to be $92 \%$. Guideway receives heat $\left(\mathrm{Q}_{\mathrm{GW} / \mathrm{in}}\right)$ via the heat exchanger and heat recouperator as shown in Figure 6.1. It is assumed that $60 \%\left(=\eta_{2}\right)$ of the heat supplied to the guideway will be utilized in melting the snow $\left(\mathrm{Q}_{\mathrm{GW} / \text { loss }}\right)$ as mentioned in equation 6.1 .

Hence, $\mathrm{Q}_{\mathrm{GW} / \mathrm{loss}}=0.6 * \mathrm{Q}_{\mathrm{GW} / \mathrm{in}}$

Therefore, the remaining heat $\left(\mathrm{Q}_{\mathrm{GW} / \mathrm{out}}\right)$ is equal to $40 \%\left(=\eta_{3}\right)$ of the heat supplied to the guideway as shown in equation 6.2.

$$
\mathrm{Q}_{\mathrm{GW} / \text { out }}=0.4 * \mathrm{Q}_{\mathrm{GW} / \text { in }}
$$


It is assumed that $40 \%$ of remaining heat $\left(\mathrm{Q}_{\mathrm{GW} / \mathrm{out}}\right)$, which is $\left(=\mathrm{Q}_{6}\right)$, will be lost to sides and bottom of guideway and the rest will be the heat re-circulated back into the heat exchanger $\left(\mathrm{Q}_{\mathrm{RC} / \mathrm{HE}}\right)$ as mentioned in equation 6.3.

$$
\mathrm{Q}_{\mathrm{RC} / \mathrm{HE}}=0.6 * \mathrm{Q}_{\mathrm{GW} / \mathrm{out}}
$$

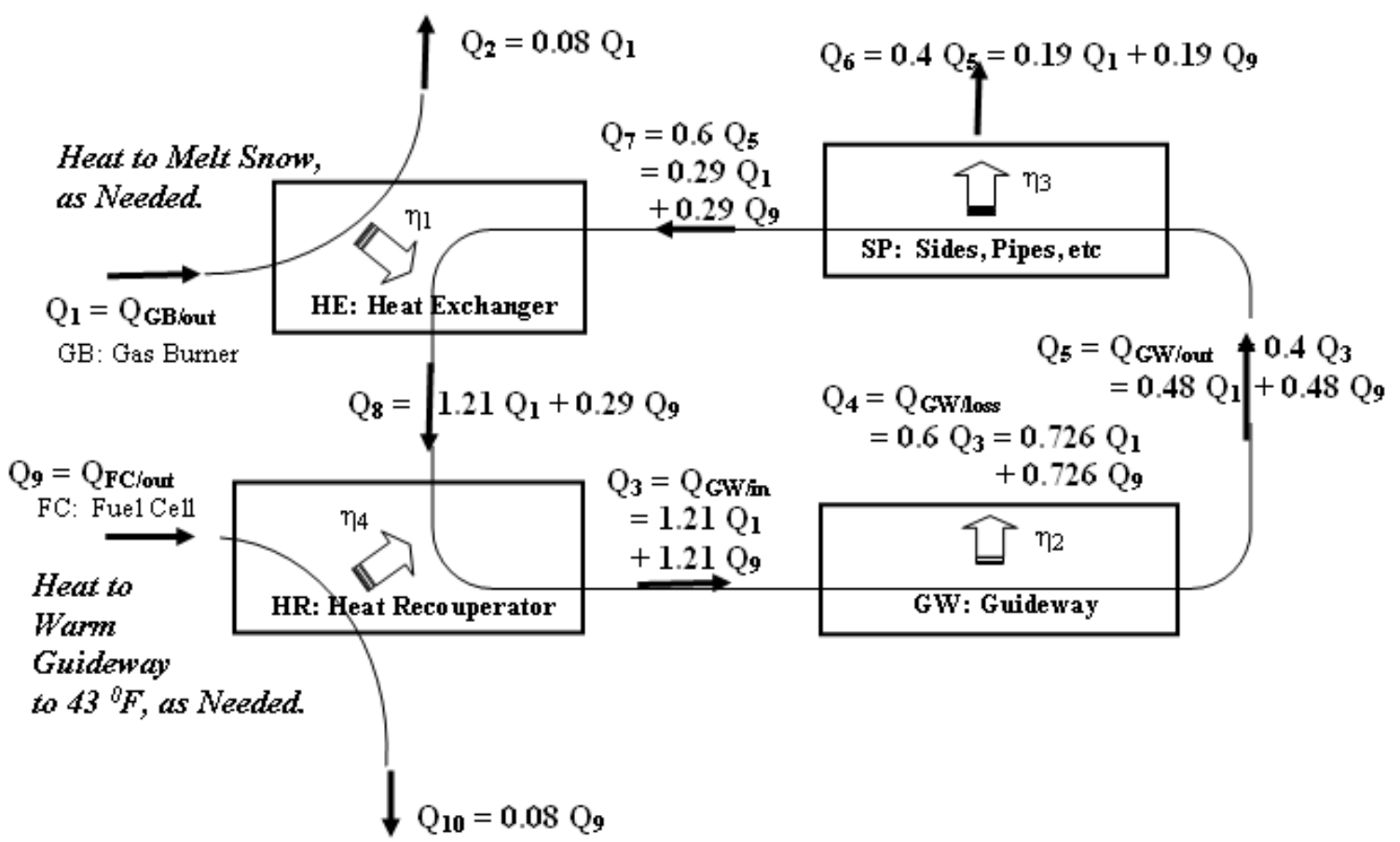

Figure 6.1: Heat Flow Diagram in Hybrid Heating System

Efficiencies of heat exchanger $\left(\eta_{1}\right)$, heat recouperator $\left(\eta_{2}\right)$, guideway $\left(\eta_{3}\right)$ and sides \& pipes $\left(\eta_{4}\right)$ will be calculated as mentioned below using equations 6.1, 6.2 and 6.3.

Definitions: HE: $\eta_{1}=\left(Q_{8}-Q_{7}\right) / Q_{1} ; \quad G W: \eta_{2}=Q_{4} / Q_{3}$;

SP: $\eta_{3}=\mathrm{Q}_{6} / \mathrm{Q}_{5} ; \quad \mathrm{HR}:\left(\mathrm{Q}_{3}-\mathrm{Q}_{8}\right) / \mathrm{Q}_{9} ;$

Energy Analyses: HE: $\mathrm{Q}_{8}=\mathrm{Q}_{7}+\eta_{1} \mathrm{Q}_{1} ; \mathrm{GW}: \mathrm{Q}_{5}=\mathrm{Q}_{3}-\mathrm{Q}_{4}$;

$$
\mathrm{SP}: \mathrm{Q}_{7}=\mathrm{Q}_{5}-\mathrm{Q}_{6} ; \mathrm{HR}: \mathrm{Q}_{3}=\mathrm{Q}_{8}+\eta_{4} \mathrm{Q}_{9} \text {; }
$$

Using the above definitions and energy analyses for Heat Exchanger (HE), Heat Recouperator (HR), Guideway (GW) and Sides, Pipes, etc (SP), the following equations are obtained as

$$
\begin{aligned}
& \text { GW: } Q_{4}=\eta_{2} Q_{3} \\
& \text { and } Q_{5}=\left(1 / \eta_{2}-1\right) Q_{4} \\
& \text { HE: } Q_{8}=Q_{7}+\eta_{1} Q_{1} \\
& \text { SP: } Q_{7}=\left(1-\eta_{3}\right) Q_{5}
\end{aligned}
$$




$$
\mathrm{HR}: \mathrm{Q}_{3}=\mathrm{Q}_{8}+\eta_{4} \mathrm{Q}_{9}
$$

Thus, using the five equations 6.4, 6.5, 6.6, 6.7 and 6.8 to solve for $\mathrm{Q}_{4}=\mathrm{f}\left(\mathrm{Q}_{1}, \mathrm{Q}_{9}\right)$ yields

$$
\mathrm{Q}_{4}=\mathrm{Q}_{1}\left[\left(\eta_{1} \eta_{2}\right) /\left(1-\left(1-\eta_{2}\right)\left(1-\eta_{3}\right)\right)\right]+\mathrm{Q}_{9}\left[\left(\eta_{2} \eta_{4}\right) /\left(1-\left(1-\eta_{2}\right)\left(1-\eta_{3}\right)\right)\right]
$$

Using the values for efficiencies, $\eta_{1}=0.92, \eta_{2}=0.6, \eta_{3}=0.4$ and $\eta_{4}=0.92$

Then, $\mathrm{Q}_{4}=0.726 \mathrm{Q}_{1}+0.726 \mathrm{Q}_{9}$

\section{Nomenclature:}

$\begin{array}{ll}\mathrm{Q}_{1} & \text { Heat output from gas burner }\left(\mathrm{Q}_{\mathrm{GB} / \mathrm{out}}\right) \\ \mathrm{Q}_{2} & \text { Heat lost in heat exchanger } \\ \mathrm{Q}_{3} & \text { Heat entering into the guideway }\left(\mathrm{Q}_{\mathrm{GW} / \mathrm{in}}\right) \\ \mathrm{Q}_{4} & \text { Heat utilized by the guideway }\left(\mathrm{Q}_{\mathrm{GW} / \text { loss }}\right) \\ \mathrm{Q}_{5} & \text { Heat coming out of the guideway }\left(\mathrm{Q}_{\mathrm{GW} / \text { out }}\right) \\ \mathrm{Q}_{6} & \text { Heat lost to the sides, bottom etc of the guideway }\left(=0.4 \mathrm{Q}_{5}\right) \\ \mathrm{Q}_{7} & \text { Heat re-circulated back into the heat exchanger }\left(\mathrm{Q}_{\mathrm{RC} / \mathrm{HE}}=0.6 \mathrm{Q}_{5}\right) \\ \mathrm{Q}_{8} & \text { Heat coming out of heat exchanger } \\ \mathrm{Q}_{9} & \text { Heat output from fuel cell }\left(\mathrm{Q}_{\mathrm{FC} / \mathrm{out}}\right) \\ \mathrm{Q}_{10} & \text { Heat lost in heat recouperator }\end{array}$

\subsection{Analysis of Hybrid Heating System}

Table 6.1 shows the detailed calculations for the heat and cost required in hybrid heating system. The cost of hydrogen and natural gas are calculated for the entire year. Fuel cell is operated throughout the year producing heat and electricity. The heat available for sale and cost of fuels (hydrogen and natural gas) are calculated.

The size of fuel cell is calculated based on the maximum thermal power required from fuel cell. Maximum heat is required when the ambient temperature is least. From the data provided my M-PRT in Table 6.1, the minimum ambient temperature is $10^{\circ} \mathrm{F}$ for the dates $\operatorname{Jan} 7,2004$ and Jan 9, 2004. The heat required to maintain the guideway at $43^{\circ} \mathrm{F}$ (for Jan 7, 2004) is calculated using equations 4.1, 6.9 and 6.10 as follows:

\section{Application:}

Peak heat output was needed on January 7, 2004. The data required is mentioned below. 
- $\quad$ Rate of snow fall (s) $=0.90 \mathrm{in} / \mathrm{hr}$

- $\quad$ Duration of snow fall $(\mathrm{d})=4.5$ hours

- Ambient temperature $\left(\mathrm{t}_{\mathrm{a}}\right)=10^{\circ} \mathrm{F}$

Heat required for melting snow on the guideway $\left(\mathrm{Q}_{\mathrm{m}}\right)=\mathrm{Q}_{\mathrm{et}}+\mathrm{Q}_{\mathrm{f}}$ is calculated using equation 4.2, 4.3 and 4.4 as follows:

$$
\begin{aligned}
\mathrm{Q}_{\mathrm{et}} & =\mathrm{d} * \mathrm{~A} *\left[\mathrm{~s} * \mathrm{D} * \mathrm{C}_{\mathrm{p}} *\left(32-\mathrm{t}_{\mathrm{a}}\right) / \mathrm{C}_{\mathrm{f}}\right] \\
& =(0.90) *(4.5) *(8.91) *(0.5) *(32-10) *(38,600) /(12)=1.3 \mathrm{MBtu} \\
\mathrm{Q}_{\mathrm{f}} & =\mathrm{d}^{*} \mathrm{~A} *\left[\mathrm{~s}^{*} \mathrm{D} * \mathrm{~h}_{\mathrm{f}} / \mathrm{C}_{\mathrm{f}}\right] \\
& =(0.90)^{*}(4.5)^{*}(8.91)^{*}(144) *(38,600) /(12)=16.7 \mathrm{MBtu} \\
\mathrm{Q}_{\mathrm{m}} & =\mathrm{Q}_{\mathrm{et}}+\mathrm{Q}_{\mathrm{cp}}=1.3+16.7=18.0 \mathrm{MBtu}
\end{aligned}
$$

where, $\mathrm{Q}_{\mathrm{et}}-$ Heat required for increasing the snow temperature to $32 \mathrm{~F}$

$$
\mathrm{Q}_{\mathrm{f}}-\text { Heat of fusion to melt snow }
$$

Heat convected from guideway $\left(\mathrm{Q}_{\mathrm{c}}\right)$ is calculated using equation 4.1 as follows:

$$
\mathrm{Q}_{\mathrm{c}}=\mathrm{d}^{*} \mathrm{~A} *\left[\mathrm{~h} *\left(43-\mathrm{t}_{\mathrm{a}}\right)\right]=(4.5)^{*}(14.3) *(43-10) *(38,600)=82 \mathrm{MBtu}
$$

Heat required from gas burner $\left(\mathrm{Q}_{\mathrm{GB} / \mathrm{out}}\right)$ and heat required from fuel cell ( $\left.\mathrm{Q}_{\mathrm{FC} / \mathrm{out}}\right)$ is calculated using equations 6.9 and 6.10 as follows:

$$
\begin{aligned}
& \mathrm{Q}_{\mathrm{GB} / \mathrm{out}}=\mathrm{Q}_{\mathrm{m}} / 0.726=18 / 0.726=24.8 \mathrm{MBtu} \\
& \mathrm{Q}_{\mathrm{FC} / \text { out }}=\mathrm{Q}_{\mathrm{c}} / 0.726=82 / 0.726=113 \mathrm{MBtu}
\end{aligned}
$$

Hence, the maximum power required by fuel cell is calculated as

$$
\mathrm{Q}_{\mathrm{FC} / \mathrm{out}} / \mathrm{d}=113 / 4.5=25 \mathrm{MBtu} / \mathrm{hr} \text {. }
$$

\section{Nomenclature:}

$$
\begin{array}{lll}
\text { Variables } & \mathrm{S} & \text { rate of snow fall }(\mathrm{in} / \mathrm{hr}) ; \\
& \mathrm{d} & \text { duration of snow fall }(\mathrm{hr}) ; \\
\text { Constants } & \mathrm{t}_{\mathrm{a}} & \text { ambient temperature }\left({ }^{\circ} \mathrm{F}\right) ; \\
& \mathrm{h}_{\mathrm{f}} & \text { heat of fusion of snow }(\mathrm{Btu} / \mathrm{lb})=144 \\
\mathrm{D} & \text { density of snow }(\mathrm{lb} / \mathrm{cuft})=8.91 \\
\mathrm{~A} & \text { surface area of heated guideway }(\mathrm{sqft})=38,600 \\
\mathrm{C}_{1} & \text { conversion factor }(\mathrm{in} / \mathrm{ft})=12 \\
\mathrm{C}_{\mathrm{p}} & \text { specific heat of snow }\left(\mathrm{Btu} / \mathrm{lb}-{ }^{\circ} \mathrm{F}\right)=0.5 \\
\mathrm{~h} & \text { convection heat transfer coefficient for air }\left(\mathrm{Btu} / \mathrm{hr}-{ }^{\circ} \mathrm{F}-\mathrm{sqft}\right)=14.3
\end{array}
$$


Table 6.1: Analysis of the Hybrid Heating System [8]

\begin{tabular}{|c|c|c|c|c|c|c|c|c|c|c|c|}
\hline $\begin{array}{l}\text { Calendar } \\
\text { Date }\end{array}$ & $\begin{array}{l}\text { Ambient } \\
\text { Temp } \\
\mathrm{T}\left({ }^{\circ} \mathrm{F}\right)\end{array}$ & $\begin{array}{c}\text { Duration } \\
\text { of } \\
\text { Snowfall } \\
\text { D (hr) }\end{array}$ & $\begin{array}{l}\text { Rate of } \\
\text { Snowfall } \\
\text { R (in/hr) }\end{array}$ & $\begin{array}{c}\text { Heat to } \\
\text { Melt } \\
\text { Snow } \\
\text { Qm } \\
\text { (MBtu) }\end{array}$ & $\begin{array}{l}\text { Convection } \\
\text { Heat Loss } \\
\text { Qc (MBtu) }\end{array}$ & $\begin{array}{c}\text { Heat } \\
\text { from } \\
\text { Gas } \\
\text { Burner } \\
\text { QGB } \\
\text { (MBtu) }\end{array}$ & $\begin{array}{l}\text { Heat } \\
\text { required } \\
\text { from } \\
\text { Fuel } \\
\text { Cell } \\
\quad \text { QFC } \\
\text { (MBtu) } \\
\end{array}$ & $\begin{array}{c}\text { Heat } \\
\text { available } \\
\text { for sale } \\
\text { (MBtu) }\end{array}$ & $\begin{array}{c}\text { Cost } \\
\text { for } \\
\text { Natural } \\
\text { Gas } \\
\text { CNG(\$) }\end{array}$ & $\begin{array}{l}\text { Cost for } \\
\text { Hydrogen } \\
\mathrm{CH}_{2}(\$)\end{array}$ & $\begin{array}{c}\text { Ratings } \\
\text { for FC } \\
\text { MBtu/hr }\end{array}$ \\
\hline \multirow[t]{2}{*}{$11 / 29 / 2003$} & 26.9 & 18.8 & 0.0 & 0.0 & 166.7 & 0.0 & 230 & 359 & $\$ 0$ & $\$ 12,300$ & 12.2 \\
\hline & 29.0 & 5.2 & 0.5 & 10.2 & 40.1 & 14.0 & 55 & 108 & $\$ 96$ & $\$ 3,400$ & 10.6 \\
\hline $11 / 30 / 2003$ & 29.0 & 24 & 0.0 & 0.0 & 185.1 & 0.0 & 255 & 496 & $\$ 0$ & $\$ 15,600$ & 10.6 \\
\hline $12 / 1 / 2003$ & 29.0 & 24 & 0.0 & 0.0 & 185.1 & 0.0 & 255 & 496 & $\$ 0$ & $\$ 15,600$ & 10.6 \\
\hline $12 / 2 / 2003$ & 29.0 & 24 & 0.0 & 0.0 & 185.1 & 0.0 & 255 & 496 & $\$ 0$ & $\$ 15,600$ & 10.6 \\
\hline $12 / 3 / 2003$ & 29.0 & 24 & 0.0 & 0.0 & 185.1 & 0.0 & 255 & 496 & $\$ 0$ & $\$ 15,600$ & 10.6 \\
\hline $12 / 4 / 2003$ & 29.0 & 24 & 0.0 & 0.0 & 185.1 & 0.0 & 255 & 496 & $\$ 0$ & $\$ 15,600$ & 10.6 \\
\hline \multirow[t]{3}{*}{$12 / 5 / 2003$} & 26.9 & 17.4 & 0.0 & 0.0 & 154.3 & 0.0 & 213 & 332 & $\$ 0$ & $\$ 11,300$ & 12.2 \\
\hline & 36.0 & 2.0 & 0.4 & 2.9 & 7.7 & 4.0 & 11 & 52 & $\$ 28$ & $\$ 1,300$ & 5.3 \\
\hline & 35.0 & 1.3 & 0.4 & 2.1 & 5.7 & 2.8 & 8 & 33 & $\$ 20$ & $\$ 800$ & 6.1 \\
\hline \multirow[t]{3}{*}{$12 / 6 / 2003$} & 26.9 & 20.4 & 0.0 & 0.0 & 180.9 & 0.0 & 249 & 389 & $\$ 0$ & $\$ 13,300$ & 12.2 \\
\hline & 34.0 & 2.4 & 0.4 & 3.9 & 11.9 & 5.4 & 16 & 59 & $\$ 37$ & $\$ 1,600$ & 6.8 \\
\hline & 28.0 & 1.2 & 0.5 & 2.4 & 9.9 & 3.3 & 14 & 24 & $\$ 23$ & $\$ 800$ & 11.4 \\
\hline $12 / 7 / 2003$ & 26.9 & 24 & 0.0 & 0.0 & 212.8 & 0.0 & 293 & 458 & $\$ 0$ & $\$ 15,600$ & 12.2 \\
\hline $12 / 8 / 2003$ & 26.9 & 24 & 0.0 & 0.0 & 212.8 & 0.0 & 293 & 458 & $\$ 0$ & $\$ 15,600$ & 12.2 \\
\hline $12 / 9 / 2003$ & 26.9 & 24 & 0.0 & 0.0 & 212.8 & 0.0 & 293 & 458 & $\$ 0$ & $\$ 15,600$ & 12.2 \\
\hline $12 / 10 / 2003$ & 26.9 & 24 & 0.0 & 0.0 & 212.8 & 0.0 & 293 & 458 & $\$ 0$ & $\$ 15,600$ & 12.2 \\
\hline $12 / 11 / 2003$ & 26.9 & 24 & 0.0 & 0.0 & 212.8 & 0.0 & 293 & 458 & $\$ 0$ & $\$ 15,600$ & 12.2 \\
\hline $12 / 12 / 2003$ & 26.9 & 24 & 0.0 & 0.0 & 212.8 & 0.0 & 293 & 458 & $\$ 0$ & $\$ 15,600$ & 12.2 \\
\hline $12 / 13 / 2003$ & 26.9 & 24 & 0.0 & 0.0 & 212.8 & 0.0 & 293 & 458 & $\$ 0$ & $\$ 15,600$ & 12.2 \\
\hline $12 / 14 / 2003$ & 26.9 & 24 & 0.0 & 0.0 & 212.8 & 0.0 & 293 & 458 & $\$ 0$ & $\$ 15,600$ & 12.2 \\
\hline $12 / 15 / 2003$ & 26.9 & 24 & 0.0 & 0.0 & 212.8 & 0.0 & 293 & 458 & $\$ 0$ & $\$ 15,600$ & 12.2 \\
\hline
\end{tabular}




\begin{tabular}{|c|c|c|c|c|c|c|c|c|c|c|c|}
\hline $12 / 17 / 2003$ & 26.9 & 24 & 0.0 & 0.0 & 212.8 & 0.0 & 293 & 458 & $\$ 0$ & $\$ 15,600$ & 12.2 \\
\hline $12 / 18 / 2003$ & 26.9 & 24 & 0.0 & 0.0 & 212.8 & 0.0 & 293 & 458 & $\$ 0$ & $\$ 15,600$ & 12.2 \\
\hline $12 / 19 / 2003$ & 26.9 & 24 & 0.0 & 0.0 & 212.8 & 0.0 & 293 & 458 & $\$ 0$ & $\$ 15,600$ & 12.2 \\
\hline $12 / 21 / 2003$ & 26.9 & 24 & 0.0 & 0.0 & 212.8 & 0.0 & 293 & 458 & $\$ 0$ & $\$ 15,600$ & 12.2 \\
\hline $12 / 22 / 2003$ & 26.9 & 24 & 0.0 & 0.0 & 212.8 & 0.0 & 293 & 458 & $\$ 0$ & $\$ 15,600$ & 12.2 \\
\hline $12 / 23 / 2003$ & 26.9 & 24 & 0.0 & 0.0 & 212.8 & 0.0 & 293 & 458 & $\$ 0$ & $\$ 15,600$ & 12.2 \\
\hline $12 / 24 / 2003$ & 26.9 & 24 & 0.0 & 0.0 & 212.8 & 0.0 & 293 & 458 & $\$ 0$ & $\$ 15,600$ & 12.2 \\
\hline $12 / 25 / 2003$ & 26.9 & 24 & 0.0 & 0.0 & 212.8 & 0.0 & 293 & 458 & $\$ 0$ & $\$ 15,600$ & 12.2 \\
\hline $12 / 26 / 2003$ & 26.9 & 24 & 0.0 & 0.0 & 212.8 & 0.0 & 293 & 458 & $\$ 0$ & $\$ 15,600$ & 12.2 \\
\hline $12 / 27 / 2003$ & 26.9 & 24 & 0.0 & 0.0 & 212.8 & 0.0 & 293 & 458 & $\$ 0$ & $\$ 15,600$ & 12.2 \\
\hline $12 / 28 / 2003$ & 26.9 & 24 & 0.0 & 0.0 & 212.8 & 0.0 & 293 & 458 & $\$ 0$ & $\$ 15,600$ & 12.2 \\
\hline $12 / 29 / 2003$ & 26.9 & 24 & 0.0 & 0.0 & 212.8 & 0.0 & 293 & 458 & $\$ 0$ & $\$ 15,600$ & 12.2 \\
\hline $12 / 30 / 2003$ & 26.9 & 24 & 0.0 & 0.0 & 212.8 & 0.0 & 293 & 458 & $\$ 0$ & $\$ 15,600$ & 12.2 \\
\hline $1 / 1 / 2004$ & 22.1 & 24 & 0.0 & 0.0 & 276.3 & 0.0 & 381 & 371 & $\$ 0$ & $\$ 15,600$ & 15.9 \\
\hline $1 / 2 / 2004$ & 22.1 & 24 & 0.0 & 0.0 & 276.3 & 0.0 & 381 & 371 & $\$ 0$ & $\$ 15,600$ & 15.9 \\
\hline $1 / 3 / 2004$ & 22.1 & 24 & 0.0 & 0.0 & 276.3 & 0.0 & 381 & 371 & $\$ 0$ & $\$ 15,600$ & 15.9 \\
\hline $1 / 4 / 2004$ & 22.1 & 24 & 0.0 & 0.0 & 276.3 & 0.0 & 381 & 371 & $\$ 0$ & $\$ 15,600$ & 15.9 \\
\hline \multirow[t]{3}{*}{$1 / 5 / 2004$} & 22.1 & 20.0 & 0.0 & 0.0 & 230.2 & 0.0 & 317 & 309 & $\$ 0$ & $\$ 13,000$ & 15.9 \\
\hline & 28.0 & 2.3 & 0.5 & 4.6 & 19.0 & 6.3 & 26 & 46 & $\$ 44$ & $\$ 1,500$ & 11.4 \\
\hline & 28.0 & 1.7 & 0.5 & 3.4 & 14.0 & 4.7 & 19 & 34 & $\$ 32$ & $\$ 1,100$ & 11.4 \\
\hline \multirow[t]{3}{*}{$1 / 6 / 2004$} & 22.1 & 18.8 & 0.0 & 0.0 & 216.4 & 0.0 & 298 & 290 & $\$ 0$ & $\$ 12,300$ & 15.9 \\
\hline & 33.0 & 2.7 & 0.4 & 4.4 & 14.8 & 6.1 & 20 & 64 & $\$ 42$ & $\$ 1,800$ & 7.6 \\
\hline & 31.0 & 2.5 & 0.4 & 4.3 & 16.5 & 6.0 & 23 & 55 & $\$ 41$ & $\$ 1,600$ & 9.1 \\
\hline \multirow[t]{2}{*}{$1 / 7 / 2004$} & 22.1 & 19.5 & 0.0 & 0.0 & 224.5 & 0.0 & 309 & 301 & $\$ 0$ & $\$ 12,700$ & 15.9 \\
\hline & 10.0 & 4.5 & 0.9 & 17.9 & 81.8 & 24.7 & 113 & 28 & $\$ 170$ & $\$ 2,900$ & 25.0 \\
\hline $1 / 8 / 2004$ & 22.1 & 18.3 & 0.0 & 0.0 & 210.7 & 0.0 & 290 & 283 & $\$ 0$ & $\$ 11,900$ & 15.9 \\
\hline
\end{tabular}




\begin{tabular}{|c|c|c|c|c|c|c|c|c|c|c|c|}
\hline $1 / 9 / 2004$ & 22.1 & 21.3 & 0.0 & 0.0 & 245.2 & 0.0 & 338 & 329 & $\$ 0$ & $\$ 13,900$ & 15.9 \\
\hline & 27.0 & 1.8 & 0.5 & 3.5 & 15.9 & 4.9 & 22 & 34 & $\$ 34$ & $\$ 1,200$ & 12.1 \\
\hline & 10.0 & 0.9 & 0.5 & 1.8 & 16.4 & 2.5 & 23 & 6 & $\$ 17$ & $\$ 600$ & 25.0 \\
\hline \multirow[t]{2}{*}{$1 / 10 / 2004$} & 22.1 & 20.2 & 0.0 & 0.0 & 232.5 & 0.0 & 320 & 312 & $\$ 0$ & $\$ 13,200$ & 15.9 \\
\hline & 22.1 & 3.8 & 0.9 & 14.6 & 43.7 & 20.0 & 60 & 59 & $\$ 138$ & $\$ 2,500$ & 15.9 \\
\hline $1 / 11 / 2004$ & 22.1 & 24 & 0.0 & 0.0 & 276.3 & 0.0 & 381 & 371 & $\$ 0$ & $\$ 15,600$ & 15.9 \\
\hline $1 / 12 / 2004$ & 22.1 & 24 & 0.0 & 0.0 & 276.3 & 0.0 & 381 & 371 & $\$ 0$ & $\$ 15,600$ & 15.9 \\
\hline \multirow[t]{3}{*}{$1 / 13 / 2004$} & 22.1 & 19.3 & 0.0 & 0.0 & 222.2 & 0.0 & 306 & 298 & $\$ 0$ & $\$ 12,600$ & 15.9 \\
\hline & 37.0 & 1.8 & 0.4 & 2.5 & 5.9 & 3.5 & 8 & 48 & $\$ 24$ & $\$ 1,200$ & 4.6 \\
\hline & 32.0 & 2.9 & 0.4 & 4.9 & 17.6 & 6.7 & 24 & 67 & $\$ 46$ & $\$ 1,900$ & 8.3 \\
\hline \multirow[t]{3}{*}{$1 / 14 / 2004$} & 22.1 & 19.9 & 0.0 & 0.0 & 229.1 & 0.0 & 316 & 307 & $\$ 0$ & $\$ 13,000$ & 15.9 \\
\hline & 26.0 & 1.7 & 0.5 & 3.3 & 15.9 & 4.5 & 22 & 31 & $\$ 31$ & $\$ 1,100$ & 12.9 \\
\hline & 35.0 & 2.4 & 0.4 & 3.8 & 10.6 & 5.2 & 15 & 61 & $\$ 36$ & $\$ 1,600$ & 6.1 \\
\hline \multirow[t]{4}{*}{$1 / 15 / 2004$} & 22.1 & 18.7 & 0.0 & 0.0 & 215.3 & 0.0 & 297 & 289 & $\$ 0$ & $\$ 12,200$ & 15.9 \\
\hline & 38.0 & 2.5 & 0.3 & 3.4 & 6.9 & 4.7 & 9 & 69 & $\$ 33$ & $\$ 1,600$ & 3.8 \\
\hline & 28.0 & 1.5 & 0.5 & 3.0 & 12.4 & 4.1 & 17 & 30 & $\$ 29$ & $\$ 1,000$ & 11.4 \\
\hline & 27.0 & 1.3 & 0.3 & 1.8 & 11.5 & 2.5 & 16 & 25 & $\$ 18$ & $\$ 800$ & 12.1 \\
\hline $1 / 16 / 2004$ & 22.1 & 24 & 0.0 & 0.0 & 276.3 & 0.0 & 381 & 371 & $\$ 0$ & $\$ 15,600$ & 15.9 \\
\hline \multirow[t]{2}{*}{$1 / 17 / 2004$} & 22.1 & 17.2 & 0.0 & 0.0 & 198.0 & 0.0 & 273 & 266 & $\$ 0$ & $\$ 11,200$ & 15.9 \\
\hline & 16.0 & 6.8 & 0.5 & 13.9 & 101.1 & 19.1 & 139 & 74 & $\$ 132$ & $\$ 4,400$ & 20.5 \\
\hline $1 / 18 / 2004$ & 22.1 & 24 & 0.0 & 0.0 & 276.3 & 0.0 & 381 & 371 & $\$ 0$ & $\$ 15,600$ & 15.9 \\
\hline $1 / 19 / 2004$ & 22.1 & 24 & 0.0 & 0.0 & 276.3 & 0.0 & 381 & 371 & $\$ 0$ & $\$ 15,600$ & 15.9 \\
\hline \multirow[t]{3}{*}{$1 / 20 / 2004$} & 22.1 & 20.4 & 0.0 & 0.0 & 234.8 & 0.0 & 323 & 315 & $\$ 0$ & $\$ 13,300$ & 15.9 \\
\hline & 19.0 & 1.4 & 0.9 & 5.1 & 18.5 & 7.0 & 25 & 18 & $\$ 49$ & $\$ 900$ & 18.2 \\
\hline & 18.0 & 2.2 & 0.9 & 8.1 & 30.3 & 11.1 & 42 & 27 & $\$ 77$ & $\$ 1,400$ & 19.0 \\
\hline \multirow[t]{2}{*}{$1 / 21 / 2004$} & 22.1 & 18.9 & 0.0 & 0.0 & 217.6 & 0.0 & 300 & 292 & $\$ 0$ & $\$ 12,300$ & 15.9 \\
\hline & 18.0 & 5.1 & 0.9 & 18.7 & 70.2 & 25.8 & 97 & 63 & $\$ 178$ & $\$ 3,300$ & 19.0 \\
\hline \multirow[t]{3}{*}{$1 / 22 / 2004$} & 22.1 & 17.6 & 0.0 & 0.0 & 202.6 & 0.0 & 279 & 272 & $\$ 0$ & $\$ 11,500$ & 15.9 \\
\hline & 25.0 & 1.7 & 0.8 & 5.4 & 16.9 & 7.5 & 23 & 30 & $\$ 52$ & $\$ 1,100$ & 13.7 \\
\hline & 25.0 & 1.8 & 0.8 & 6.1 & 17.8 & 8.4 & 25 & 32 & $\$ 58$ & $\$ 1,200$ & 13.7 \\
\hline
\end{tabular}




\begin{tabular}{|c|c|c|c|c|c|c|c|c|c|c|c|}
\hline & 25.0 & 2.9 & 0.4 & 5.4 & 28.8 & 7.4 & 40 & 51 & $\$ 51$ & $\$ 1,900$ & 13.7 \\
\hline \multirow[t]{5}{*}{$1 / 23 / 2004$} & 22.1 & 11.3 & 0.0 & 0.0 & 130.1 & 0.0 & 179 & 174 & $\$ 0$ & $\$ 7,400$ & 15.9 \\
\hline & 25.0 & 1.6 & 0.8 & 5.4 & 15.9 & 7.4 & 22 & 28 & $\$ 51$ & $\$ 1,000$ & 13.7 \\
\hline & 12.0 & 2.7 & 0.4 & 5.1 & 46.1 & 7.0 & 64 & 21 & $\$ 49$ & $\$ 1,800$ & 23.5 \\
\hline & 18.0 & 5.9 & 0.4 & 11.2 & 81.2 & 15.4 & 112 & 73 & $\$ 106$ & $\$ 3,800$ & 19.0 \\
\hline & 18.0 & 2.5 & 0.9 & 9.3 & 34.4 & 12.8 & 47 & 31 & $\$ 88$ & $\$ 1,600$ & 19.0 \\
\hline \multirow[t]{3}{*}{$1 / 24 / 2004$} & 22.1 & 16.7 & 0.0 & 0.0 & 192.3 & 0.0 & 265 & 258 & $\$ 0$ & $\$ 10,900$ & 15.9 \\
\hline & 21.0 & 5.9 & 0.8 & 20.2 & 71.5 & 27.8 & 98 & 86 & $\$ 191$ & $\$ 3,800$ & 16.7 \\
\hline & 20.0 & 1.4 & 0.8 & 4.8 & 17.7 & 6.6 & 24 & 19 & $\$ 46$ & $\$ 900$ & 17.5 \\
\hline \multirow[t]{3}{*}{$1 / 25 / 2004$} & 22.1 & 13.5 & 0.0 & 0.0 & 155.4 & 0.0 & 214 & 208 & $\$ 0$ & $\$ 8,800$ & 15.9 \\
\hline & 17.0 & 6.6 & 0.5 & 14.3 & 94.5 & 19.7 & 130 & 76 & $\$ 136$ & $\$ 4,300$ & 19.7 \\
\hline & 12.0 & 3.9 & 0.5 & 8.6 & 66.6 & 11.8 & 92 & 30 & $\$ 81$ & $\$ 2,500$ & 23.5 \\
\hline \multirow[t]{3}{*}{$1 / 26 / 2004$} & 22.1 & 19.6 & 0.0 & 0.0 & 225.6 & 0.0 & 311 & 303 & $\$ 0$ & $\$ 12,800$ & 15.9 \\
\hline & 27.0 & 1.1 & 0.8 & 3.7 & 9.7 & 5.1 & 13 & 21 & $\$ 35$ & $\$ 700$ & 12.1 \\
\hline & 20.0 & 2.0 & 0.4 & 3.6 & 25.3 & 5.0 & 35 & 28 & $\$ 34$ & $\$ 1,300$ & 17.5 \\
\hline \multirow[t]{5}{*}{$1 / 27 / 2004$} & 22.1 & 16.4 & 0.0 & 0.0 & 188.8 & 0.0 & 260 & 253 & $\$ 0$ & $\$ 10,700$ & 15.9 \\
\hline & 32.0 & 1.9 & 0.5 & 3.9 & 11.5 & 5.4 & 16 & 44 & $\$ 37$ & $\$ 1,200$ & 8.3 \\
\hline & 30.0 & 3.1 & 0.5 & 6.0 & 22.2 & 8.3 & 31 & 66 & $\$ 57$ & $\$ 2,000$ & 9.9 \\
\hline & 22.0 & 0.6 & 0.4 & 1.1 & 6.9 & 1.5 & 10 & 9 & $\$ 11$ & $\$ 400$ & 15.9 \\
\hline & 29.0 & 2.0 & 0.5 & 3.7 & 15.4 & 5.2 & 21 & 41 & $\$ 36$ & $\$ 1,300$ & 10.6 \\
\hline \multirow[t]{3}{*}{ 1/28/2004 } & 22.1 & 17.4 & 0.0 & 0.0 & 200.3 & 0.0 & 276 & 269 & $\$ 0$ & $\$ 11,300$ & 15.9 \\
\hline & 22.0 & 1.5 & 0.4 & 2.8 & 17.4 & 3.9 & 24 & 23 & $\$ 27$ & $\$ 1,000$ & 15.9 \\
\hline & 18.0 & 5.1 & 0.5 & 9.9 & 70.2 & 13.6 & 97 & 63 & $\$ 94$ & $\$ 3,300$ & 19.0 \\
\hline \multirow[t]{2}{*}{$1 / 29 / 2004$} & 22.1 & 14.2 & 0.0 & 0.0 & 163.5 & 0.0 & 225 & 219 & $\$ 0$ & $\$ 9,300$ & 15.9 \\
\hline & 24.0 & 3.1 & 0.7 & 9.2 & 32.4 & 12.6 & 45 & 52 & $\$ 87$ & $\$ 2,000$ & 14.4 \\
\hline
\end{tabular}




\begin{tabular}{|c|c|c|c|c|c|c|c|c|c|c|c|}
\hline $1 / 30 / 2004$ & 22.1 & 15.6 & 0.0 & 0.0 & 179.6 & 0.0 & 247 & 241 & $\$ 0$ & $\$ 10,200$ & 15.9 \\
\hline & 20.0 & 5.5 & 0.5 & 12.3 & 69.7 & 16.9 & 96 & 76 & $\$ 116$ & $\$ 3,600$ & 17.5 \\
\hline & 15.0 & 2.9 & 0.9 & 10.7 & 44.7 & 14.8 & 62 & 29 & $\$ 102$ & $\$ 1,900$ & 21.2 \\
\hline $1 / 31 / 2004$ & 22.1 & 24 & 0.0 & 0.0 & 276.3 & 0.0 & 381 & 371 & $\$ 0$ & $\$ 15,600$ & 15.9 \\
\hline 2/1/2004 & 24.2 & 24 & 0.0 & 0.0 & 248.5 & 0.0 & 342 & 409 & $\$ 0$ & $\$ 15,600$ & 14.3 \\
\hline $2 / 2 / 2004$ & 24.2 & 24 & 0.0 & 0.0 & 248.5 & 0.0 & 342 & 409 & $\$ 0$ & $\$ 15,600$ & 14.3 \\
\hline $2 / 3 / 2004$ & 24.2 & 24 & 0.0 & 0.0 & 248.5 & 0.0 & 342 & 409 & $\$ 0$ & $\$ 15,600$ & 14.3 \\
\hline 2/4/2004 & 24.2 & 24 & 0.0 & 0.0 & 248.5 & 0.0 & 342 & 409 & $\$ 0$ & $\$ 15,600$ & 14.3 \\
\hline $2 / 5 / 2004$ & 24.2 & 24 & 0.0 & 0.0 & 248.5 & 0.0 & 342 & 409 & $\$ 0$ & $\$ 15,600$ & 14.3 \\
\hline $2 / 6 / 2004$ & 24.2 & 24 & 0.0 & 0.0 & 248.5 & 0.0 & 342 & 409 & $\$ 0$ & $\$ 15,600$ & 14.3 \\
\hline \multirow[t]{3}{*}{$2 / 7 / 2004$} & 24.2 & 19.2 & 0.0 & 0.0 & 198.8 & 0.0 & 274 & 327 & $\$ 0$ & $\$ 12,500$ & 14.3 \\
\hline & 33.0 & 3.8 & 0.4 & 6.2 & 20.9 & 8.6 & 29 & 90 & $\$ 59$ & $\$ 2,500$ & 7.6 \\
\hline & 32.0 & 1.0 & 0.4 & 1.7 & 6.1 & 2.3 & 8 & 23 & $\$ 16$ & $\$ 700$ & 8.3 \\
\hline $2 / 8 / 2004$ & 24.2 & 24 & 0.0 & 0.0 & 248.5 & 0.0 & 342 & 409 & $\$ 0$ & $\$ 15,600$ & 14.3 \\
\hline 2/9/2004 & 24.2 & 24 & 0.0 & 0.0 & 248.5 & 0.0 & 342 & 409 & $\$ 0$ & $\$ 15,600$ & 14.3 \\
\hline 2/10/2004 & 24.2 & 24 & 0.0 & 0.0 & 248.5 & 0.0 & 342 & 409 & $\$ 0$ & $\$ 15,600$ & 14.3 \\
\hline $2 / 11 / 2004$ & 24.2 & 24 & 0.0 & 0.0 & 248.5 & 0.0 & 342 & 409 & $\$ 0$ & $\$ 15,600$ & 14.3 \\
\hline \multirow[t]{2}{*}{$2 / 12 / 2004$} & 24.2 & 22.7 & 0.0 & 0.0 & 235.1 & 0.0 & 324 & 387 & $\$ 0$ & $\$ 14,800$ & 14.3 \\
\hline & 28.0 & 1.3 & 0.5 & 2.4 & 10.7 & 3.4 & 15 & 26 & $\$ 23$ & $\$ 800$ & 11.4 \\
\hline 2/13/2004 & 24.2 & 24 & 0.0 & 0.0 & 248.5 & 0.0 & 342 & 409 & $\$ 0$ & $\$ 15,600$ & 14.3 \\
\hline 2/14/2004 & 24.2 & 24 & 0.0 & 0.0 & 248.5 & 0.0 & 342 & 409 & $\$ 0$ & $\$ 15,600$ & 14.3 \\
\hline $2 / 15 / 2004$ & 24.2 & 24 & 0.0 & 0.0 & 248.5 & 0.0 & 342 & 409 & $\$ 0$ & $\$ 15,600$ & 14.3 \\
\hline $2 / 16 / 2004$ & 24.2 & 24 & 0.0 & 0.0 & 248.5 & 0.0 & 342 & 409 & $\$ 0$ & $\$ 15,600$ & 14.3 \\
\hline $2 / 17 / 2004$ & 24.2 & 24 & 0.0 & 0.0 & 248.5 & 0.0 & 342 & 409 & $\$ 0$ & $\$ 15,600$ & 14.3 \\
\hline 2/18/2004 & 24.2 & 24 & 0.0 & 0.0 & 248.5 & 0.0 & 342 & 409 & $\$ 0$ & $\$ 15,600$ & 14.3 \\
\hline 2/19/2004 & 24.2 & 24 & 0.0 & 0.0 & 248.5 & 0.0 & 342 & 409 & $\$ 0$ & $\$ 15,600$ & 14.3 \\
\hline $2 / 20 / 2004$ & 24.2 & 24 & 0.0 & 0.0 & 248.5 & 0.0 & 342 & 409 & $\$ 0$ & $\$ 15,600$ & 14.3 \\
\hline
\end{tabular}




\begin{tabular}{|c|c|c|c|c|c|c|c|c|c|c|c|}
\hline 2/21/2004 & 24.2 & 19.0 & 0.0 & 0.0 & 196.8 & 0.0 & 271 & 324 & $\$ 0$ & $\$ 12,400$ & 14.3 \\
\hline & 39.0 & 2.0 & 0.2 & 1.6 & 4.4 & 2.2 & 6 & 57 & $\$ 15$ & $\$ 1,300$ & 3.0 \\
\hline & 39.0 & 2.0 & 0.3 & 2.4 & 4.4 & 3.3 & 6 & 57 & $\$ 23$ & $\$ 1,300$ & 3.0 \\
\hline & 39.0 & 1.0 & 0.4 & 1.4 & 2.2 & 1.9 & 3 & 28 & $\$ 13$ & $\$ 700$ & 3.0 \\
\hline 2/22/2004 & 24.2 & 24 & 0.0 & 0.0 & 248.5 & 0.0 & 342 & 409 & $\$ 0$ & $\$ 15,600$ & 14.3 \\
\hline 2/23/2004 & 24.2 & 24 & 0.0 & 0.0 & 248.5 & 0.0 & 342 & 409 & $\$ 0$ & $\$ 15,600$ & 14.3 \\
\hline $2 / 25 / 2004$ & 24.2 & 24 & 0.0 & 0.0 & 248.5 & 0.0 & 342 & 409 & $\$ 0$ & $\$ 15,600$ & 14.3 \\
\hline $2 / 26 / 2004$ & 24.2 & 24 & 0.0 & 0.0 & 248.5 & 0.0 & 342 & 409 & $\$ 0$ & $\$ 15,600$ & 14.3 \\
\hline $2 / 27 / 2004$ & 24.2 & 24 & 0.0 & 0.0 & 248.5 & 0.0 & 342 & 409 & $\$ 0$ & $\$ 15,600$ & 14.3 \\
\hline 2/28/2004 & 24.2 & 24 & 0.0 & 0.0 & 248.5 & 0.0 & 342 & 409 & $\$ 0$ & $\$ 15,600$ & 14.3 \\
\hline 2/29/2004 & 24.2 & 24 & 0.0 & 0.0 & 248.5 & 0.0 & 342 & 409 & $\$ 0$ & $\$ 15,600$ & 14.3 \\
\hline $3 / 2 / 2004$ & 31.8 & 24 & 0.0 & 0.0 & 148.1 & 0.0 & 204 & 547 & $\$ 0$ & $\$ 15,600$ & 8.5 \\
\hline $3 / 3 / 2004$ & 31.8 & 24 & 0.0 & 0.0 & 148.1 & 0.0 & 204 & 547 & $\$ 0$ & $\$ 15,600$ & 8.5 \\
\hline $3 / 4 / 2004$ & 31.8 & 24 & 0.0 & 0.0 & 148.1 & 0.0 & 204 & 547 & $\$ 0$ & $\$ 15,600$ & 8.5 \\
\hline $3 / 5 / 2004$ & 31.8 & 24 & 0.0 & 0.0 & 148.1 & 0.0 & 204 & 547 & $\$ 0$ & $\$ 15,600$ & 8.5 \\
\hline $3 / 6 / 2004$ & 31.8 & 24 & 0.0 & 0.0 & 148.1 & 0.0 & 204 & 547 & $\$ 0$ & $\$ 15,600$ & 8.5 \\
\hline $3 / 7 / 2004$ & 31.8 & 24 & 0.0 & 0.0 & 148.1 & 0.0 & 204 & 547 & $\$ 0$ & $\$ 15,600$ & 8.5 \\
\hline \multirow[t]{5}{*}{$3 / 8 / 2004$} & 31.8 & 14.9 & 0.0 & 0.0 & 91.9 & 0.0 & 127 & 340 & $\$ 0$ & $\$ 9,700$ & 8.5 \\
\hline & 37.0 & 2.7 & 0.4 & 3.8 & 8.9 & 5.3 & 12 & 72 & $\$ 36$ & $\$ 1,800$ & 4.6 \\
\hline & 34.0 & 2.0 & 0.4 & 3.0 & 9.9 & 4.2 & 14 & 49 & $\$ 29$ & $\$ 1,300$ & 6.8 \\
\hline & 34.0 & 1.5 & 0.4 & 2.3 & 7.4 & 3.1 & 10 & 37 & $\$ 22$ & $\$ 1,000$ & 6.8 \\
\hline & 32.0 & 2.9 & 0.4 & 4.7 & 17.6 & 6.4 & 24 & 67 & $\$ 44$ & $\$ 1,900$ & 8.3 \\
\hline $3 / 9 / 2004$ & 31.8 & 24 & 0.0 & 0.0 & 148.1 & 0.0 & 204 & 547 & $\$ 0$ & $\$ 15,600$ & 8.5 \\
\hline
\end{tabular}




\begin{tabular}{|c|c|c|c|c|c|c|c|c|c|c|c|}
\hline $3 / 12 / 2004$ & 31.8 & 22.3 & 0.0 & 0.0 & 137.6 & 0.0 & 189 & 508 & $\$ 0$ & $\$ 14,500$ & 8.5 \\
\hline & 25.0 & 1.7 & 0.4 & 3.1 & 16.9 & 4.2 & 23 & 30 & $\$ 29$ & $\$ 1,100$ & 13.7 \\
\hline $3 / 13 / 2004$ & 31.8 & 24 & 0.0 & 0.0 & 148.1 & 0.0 & 204 & 547 & $\$ 0$ & $\$ 15,600$ & 8.5 \\
\hline $3 / 14 / 2004$ & 31.8 & 24 & 0.0 & 0.0 & 148.1 & 0.0 & 204 & 547 & $\$ 0$ & $\$ 15,600$ & 8.5 \\
\hline $3 / 15 / 2004$ & 31.8 & 24 & 0.0 & 0.0 & 148.1 & 0.0 & 204 & 547 & $\$ 0$ & $\$ 15,600$ & 8.5 \\
\hline $3 / 16 / 2004$ & 31.8 & 24 & 0.0 & 0.0 & 148.1 & 0.0 & 204 & 547 & $\$ 0$ & $\$ 15,600$ & 8.5 \\
\hline $3 / 18 / 2004$ & 31.8 & 24 & 0.0 & 0.0 & 148.1 & 0.0 & 204 & 547 & $\$ 0$ & $\$ 15,600$ & 8.5 \\
\hline $3 / 19 / 2004$ & 31.8 & 24 & 0.0 & 0.0 & 148.1 & 0.0 & 204 & 547 & $\$ 0$ & $\$ 15,600$ & 8.5 \\
\hline $3 / 20 / 2004$ & 31.8 & 24 & 0.0 & 0.0 & 148.1 & 0.0 & 204 & 547 & $\$ 0$ & $\$ 15,600$ & 8.5 \\
\hline $3 / 21 / 2004$ & 31.8 & 24 & 0.0 & 0.0 & 148.1 & 0.0 & 204 & 547 & $\$ 0$ & $\$ 15,600$ & 8.5 \\
\hline $3 / 22 / 2004$ & 31.8 & 20.8 & 0.0 & 0.0 & 128.3 & 0.0 & 177 & 474 & $\$ 0$ & $\$ 13,600$ & 8.5 \\
\hline $3 / 23 / 2004$ & 31.8 & 24 & 0.0 & 0.0 & 148.1 & 0.0 & 204 & 547 & $\$ 0$ & $\$ 15,600$ & 8.5 \\
\hline $3 / 24 / 2004$ & 31.8 & 24 & 0.0 & 0.0 & 148.1 & 0.0 & 204 & 547 & $\$ 0$ & $\$ 15,600$ & 8.5 \\
\hline $3 / 25 / 2004$ & 31.8 & 24 & 0.0 & 0.0 & 148.1 & 0.0 & 204 & 547 & $\$ 0$ & $\$ 15,600$ & 8.5 \\
\hline $3 / 26 / 2004$ & 31.8 & 24 & 0.0 & 0.0 & 148.1 & 0.0 & 204 & 547 & $\$ 0$ & $\$ 15,600$ & 8.5 \\
\hline $3 / 27 / 2004$ & 31.8 & 24 & 0.0 & 0.0 & 148.1 & 0.0 & 204 & 547 & $\$ 0$ & $\$ 15,600$ & 8.5 \\
\hline $3 / 28 / 2004$ & 31.8 & 24 & 0.0 & 0.0 & 148.1 & 0.0 & 204 & 547 & $\$ 0$ & $\$ 15,600$ & 8.5 \\
\hline $3 / 29 / 2004$ & 31.8 & 24 & 0.0 & 0.0 & 148.1 & 0.0 & 204 & 547 & $\$ 0$ & $\$ 15,600$ & 8.5 \\
\hline $3 / 30 / 2004$ & 31.8 & 24 & 0.0 & 0.0 & 148.1 & 0.0 & 204 & 547 & $\$ 0$ & $\$ 15,600$ & 8.5 \\
\hline $3 / 31 / 2004$ & 31.8 & 24 & 0.0 & 0.0 & 148.1 & 0.0 & 204 & 547 & $\$ 0$ & $\$ 15,600$ & 8.5 \\
\hline
\end{tabular}


Hence, the maximum thermal power required is $25 \mathrm{MBtu} / \mathrm{hr}$. Therefore, fuel cell should be capable of producing a minimum of $25 \mathrm{MBtu} / \mathrm{hr}$ of heat to the guideway. Fuel cell (thermal efficiency of $36 \%$ ) shall be oversized by $25 \%$ to meet extreme climatic conditions. Hence, the total power of fuel cell needed is $(25 / 0.36) * 1.25=86.8 \mathrm{MBtu} / \mathrm{Hr}$ or $25,481 \mathrm{~kW}$.

The maximum thermal power generated by fuel cell is $31 \mathrm{MBtu} / \mathrm{hr}$. Electrical efficiency of fuel cell is $40 \%$. The maximum electrical power generated by fuel cell is $(25,500 * 0.40)=10,200 \mathrm{~kW}$. The fuel cell as sized at $25,500 \mathrm{~kW}$ shall continue to produce $31 \mathrm{MBtu} / \mathrm{hr}$ of heat and 10,200 $\mathrm{kW}$ of electricity throughout the year.

Table 6.2: Heat Required and Cost of Fuel used in Existing and Hybrid Heating Systems

\begin{tabular}{|c|c|c|c|c|c|c|}
\hline MONTH & $\begin{array}{c}\text { Heat from } \\
\text { GB in } \\
\text { Existing } \\
\text { Heating } \\
\text { System }\end{array}$ & $\begin{array}{c}\text { Cost of } \\
\text { Fuel in } \\
\text { Existing } \\
\text { Heating } \\
\text { System } \\
\left(\mathrm{C}_{\mathrm{E}}\right)\end{array}$ & $\begin{array}{c}\text { Heat from } \\
\text { GB in } \\
\text { Hybrid } \\
\text { Heating } \\
\text { System }\end{array}$ & $\begin{array}{c}\text { Heat from } \\
\text { Fuel Cell } \\
\text { for } \\
\text { guideway } \\
\text { heating in } \\
\text { Hybrid } \\
\text { Heating } \\
\text { System }\end{array}$ & $\begin{array}{c}\text { Heat } \\
\text { available } \\
\text { for sale in } \\
\text { Hybrid } \\
\text { Heating } \\
\text { System }\end{array}$ & $\begin{array}{c}\text { Cost of Fuel } \\
\text { in Hybrid } \\
\text { Heating } \\
\text { System } \\
\left(\mathrm{C}_{\mathrm{H}}\right)\end{array}$ \\
\hline & (MBtu) & $(\$)$ & $(\mathrm{MBtu})$ & (MBtu) & (MBtu) & $(\$)$ \\
\hline November & 0 & 0 & 0 & 0 & 21,000 & $\$ 438,000$ \\
\hline December & 161 & $\$ 1,100$ & 37 & 9,420 & 15,400 & $\$ 515,000$ \\
\hline January & 2,420 & $\$ 16,700$ & 432 & 11,800 & 11,500 & $\$ 487,000$ \\
\hline February & 89 & $\$ 613$ & 22 & 9,800 & 11,900 & $\$ 453,000$ \\
\hline March & 146 & $\$ 1,000$ & 31 & 6,300 & 17,000 & $\$ 484,000$ \\
\hline April & 0 & 0 & 0 & 0 & 22,500 & $\$ 470,000$ \\
\hline May & 0 & 0 & 0 & 0 & 23,300 & $\$ 485,000$ \\
\hline June & 0 & 0 & 0 & 0 & 22,500 & $\$ 470,000$ \\
\hline July & 0 & 0 & 0 & 0 & 23,300 & $\$ 485,000$ \\
\hline August & 0 & 0 & 0 & 0 & 23,300 & $\$ 485,000$ \\
\hline September & 0 & 0 & 0 & 0 & 22,500 & $\$ 470,000$ \\
\hline October & 0 & 0 & 0 & 0 & 23,300 & $\$ 485,000$ \\
\hline TOTAL & $\mathbf{2 , 8 0 0}$ & $\mathbf{\$ 1 9 , 4 0 0}$ & $\mathbf{5 2 1}$ & $\mathbf{3 7 , 3 0 0}$ & $\mathbf{2 3 8 , 0 0 0}$ & $\$ 5,731,000$ \\
\hline
\end{tabular}

From Table 6.2, the cost of fuel in hybrid heating system is very high when compared to the cost of fuel in existing heating system. The cost of hybrid heating system includes the cost of fuel in fuel cell and natural gas burner. Since the fuel cell is continuously operated at maximum power throughout the year the cost of hydrogen remains constant. 
In December, February and March the fuel cost ratio between hybrid and existing heating system is much higher than January. One of the reasons being that the fuel cell is supplying heat throughout the month (including the non-snowing hours) where as the existing heating system is supplying heat only during the snowing hours. The number of snowing hours is relatively more in January when compared to other months. Hence the cost of fuel in existing heating system is very less in December, February and March when compared to January.

The other reason is that the average ambient temperature in January is lower than the average ambient temperature in other months. Hence, the increase in cost of hybrid heating system is not only dependant on the rate of snowfall or amount of snowfall but also dependant on the ambient temperature.

\subsection{Heat Generated and Fuel Used}

The heat produced and fuels used by the hybrid heater are shown in Figure 6.2. The heat produced from gas burner in hybrid heating system is less than that in existing heating system. The hybrid heating system receives major amount of heat from fuel cell. Since the guideway is maintained at $43^{\circ} \mathrm{F}$ throughout the winter, the heat required by gas burner to melt the snow reduces by more than $50 \%$.

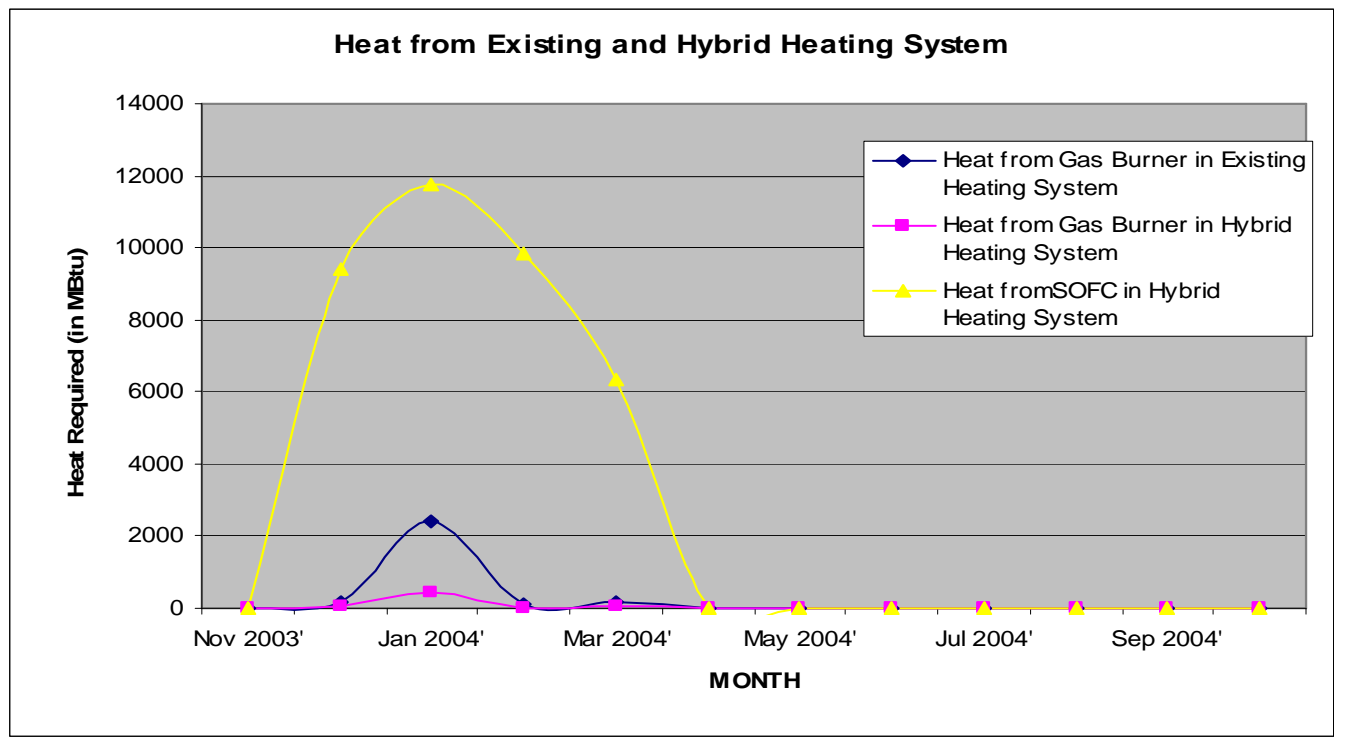

Figure 6.2: Heat Produced by Natural Gas Burner and Fuel Cell

for Hybrid Heating System 
From Table 6.2, total heat output from gas burner in existing heating system is 2,815 MBtu. Heat capacity of natural gas is $1000 \mathrm{Btu} / \mathrm{ft}^{3}$ or $1 \mathrm{MBtu} / \mathrm{MCF}$. It is assumed that $20 \%$ of natural gas may be utilized for start-ups and the efficiency of gas burner is $87 \%$. The total natural gas used in existing heating system is calculated as $1.2 *(2,800) / 0.87$ $=3,890 \mathrm{MCF}$. From Table 6.2, total heat output from gas burner in hybrid heating system is $521 \mathrm{MBtu}$. Therefore, natural gas used in hybrid guideway heating system is calculated as $1.2 *(521) / 0.87=719 \mathrm{MCF}$. The natural gas used in hybrid heating system is much lesser than that in existing heating system as shown in Figure 6.3. This is because the hybrid heating system maintains the guideway at $43^{\circ} \mathrm{F}$ using fuel cell supplied heat.

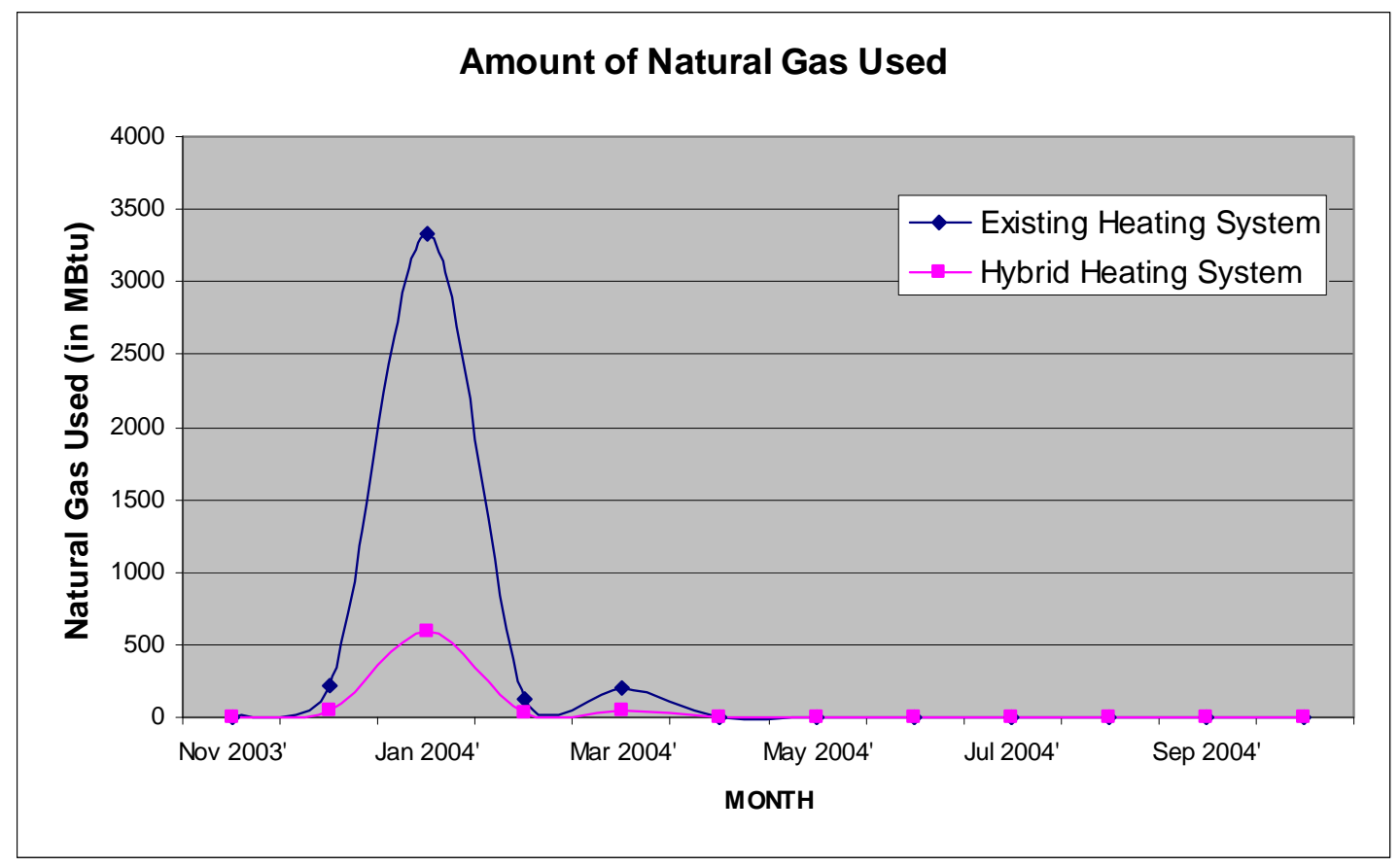

Figure 6.3: Natural Gas used in Existing and Hybrid Heating System

\subsection{Emissions Produced in Hybrid Heating System}

Emissions produced by natural gas burner are assumed to be equal to $120 \mathrm{lbs}$ per MBtu of heat produced. Heat produced by gas burner in existing heating system is 2,800 MBtu and heat produced by gas burner in hybrid heating system is 520 MBtu. Emissions 
produced by natural gas burner in existing heating system exceeds by 240,000 lbs/MBtu to that in hybrid heating system. Hybrid heating system producing fewer emissions can prove to be more eco-friendly than existing heating system as shown in Figure 6.4. Emissions produced from fuel cell are negligible when compared with the emissions from natural gas burner.

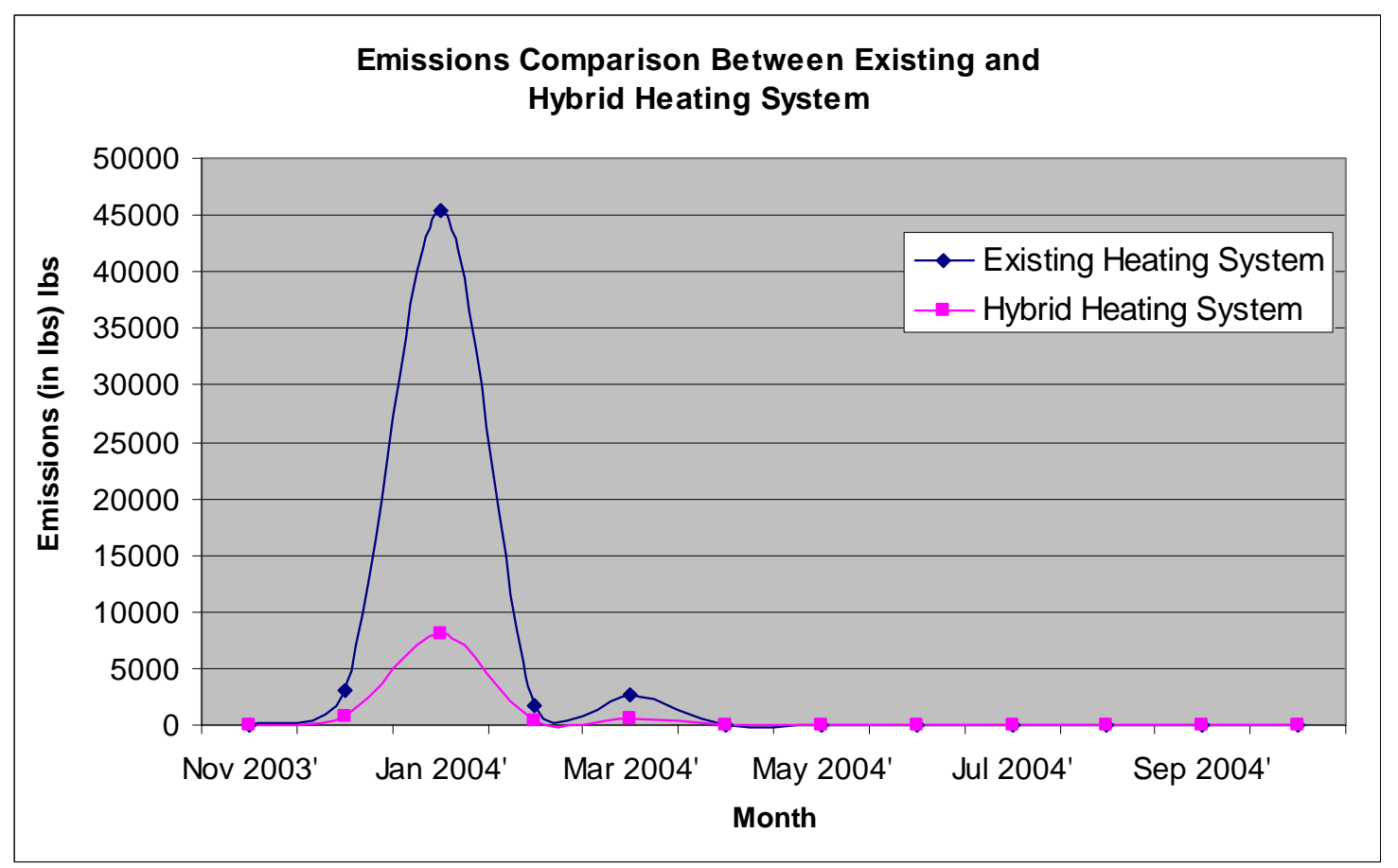

Figure 6.4: Emissions Produced in Existing and Hybrid Heating System

\subsection{Limitations for Hybrid Heating System}

In spite of several advantages, the hybrid heating system has limitations that are as follows:

a. The system uses hydrogen as one of its fuel inputs which is difficult to store and is also explosive.

b. The hybrid heating system involves high capital cost due to high purchase price of fuel cell. 


\section{Chapter 7}

\section{Economic Justification}

\subsection{Fixed and Variable Costs in Hybrid Heating System}

The heat required by the guideway during winter is calculated in the spreadsheet. The total power of fuel cell as sized at the maximum power required in hybrid heating system is $25,500 \mathrm{~kW}$.

The fixed costs in hybrid heating system includes the cost of fuel cell, heat recouperator, gas burner, heat exchanger and capital cost of selective catalytic reducer (SCR). The capital cost of producing fuel cell is assumed to be $\$ 1480$ per $\mathrm{kW}$ of total power or $\$ 3700$ per $\mathrm{kW}$ of electrical power produced.

- Capital cost of fuel cell is equal to $(25,500 * 1480)=\$ 37,712,000$

- Average cost of gas burner and heat exchanger in boiler plant 3 (BP3) is $\$ 130,000$.

- Average life of fuel cell, gas burner, heat exchanger and heat recouperator are assumed to be 20 years.

- Cost of selective catalytic reducer (SCR) is $\$ 8000$ per MBtu/hr of gas burner heat produced. Average life of SCR is assumed to be 10 years.

The variable costs in M-PRT heating systems are the costs of fuels (natural gas and hydrogen), operational and maintenance (O\&M) costs and cost to reduce emissions using SCR. Annual operational and maintenance (O\&M) cost of gas burner and heat exchanger is assumed to be $20 \%$ of its capital cost equivalent annually.

- O\&M cost for gas burner/heat exchanger in existing heating system is $\$ 26,000$ annually. O\&M cost for gas burner/heat exchanger in hybrid heating system is $\$ 6,200$ annually.

- Annual O\&M cost of fuel cell/heat recouperator is assumed to be $\$ 0.005$ per $\mathrm{kW}$ hr of electrical energy produced. O\&M cost of fuel cell/heat recouperator in hybrid heating system is $\$ 447,600$ annually. 
Table 7.1: Assessment of M-PRT Guideway Heating Systems

\begin{tabular}{|c|c|c|c|c|c|c|c|c|c|c|c|}
\hline \multicolumn{12}{|c|}{ Assessment of M-PRT Guideway Heating Systems } \\
\hline \multirow[t]{10}{*}{1} & 1.0 & Required Heat & \multicolumn{3}{|c|}{ Existing System } & \multicolumn{3}{|c|}{ Hybrid System with Thesis FC } & \multicolumn{3}{|c|}{ Hybrid System w/ Commercial FC } \\
\hline & & & & Peak & Annual & & Peak & Annual & & Peak & Annual \\
\hline & & & & MBtu/hr & MBtu & & MBtu/hr & MBtu & & MBtu/hr & MBtu \\
\hline & 1.1 & Heat Guideway & GB & 18.2 & & $\mathrm{FC}$ & 18.2 & & $\mathrm{FC}$ & 18.2 & \\
\hline & 1.2 & Guideway Losses & GB & 4.8 & & FC & 4.8 & & $\mathrm{FC}$ & 4.8 & \\
\hline & 1.3 & Heater Losses & GB & 3.5 & & FC & 2.9 & & FC & 2.9 & \\
\hline & 1.4 & Heater Losses & & & & GB & 0.6 & & GB & 0.6 & \\
\hline & 1.5 & Melt Snow & GB & 4 & & GB & 4 & & GB & 4 & \\
\hline & 1.6 & Totals & GB & 30.5 & 2815 & GB & 5.5 & 521 & GB & 5.5 & 521 \\
\hline & & & & & & FC & 25.0 & 37,338 & FC & 25.0 & 37,338 \\
\hline \multirow[t]{9}{*}{2} & 2.0 & Fixed Costs & \multicolumn{3}{|c|}{ Existing System } & \multicolumn{3}{|c|}{ Hybrid System with Thesis FC } & \multicolumn{3}{|c|}{ Hybrid System w/ Commercial FC } \\
\hline & & & & Purchase & Annual & & Purchase & Annual & & Purchase & Annual \\
\hline & & & & $\$$ & $\$$ & & $\$$ & $\$$ & & $\$$ & $\$$ \\
\hline & 2.1 & Gas Burner (GB) & & 100,000 & 5,000 & & 25,000 & 1,250 & & 25,000 & 1,250 \\
\hline & 2.2 & Heat Exchanger (HE) & & 30,000 & 1,500 & & 6,000 & 300 & & 6,000 & 300 \\
\hline & 2.3 & Fuel Cell (FC) & & 0 & 0 & $\mathrm{FC}$ & $37,712,000$ & $1,886,000$ & $\mathrm{FC}$ & $47,176,000$ & $2,359,000$ \\
\hline & 2.4 & Heat Recouperator (HR) & & 0 & 0 & $\mathrm{HR}$ & 30,000 & 1,500 & $\mathrm{HR}$ & 30,000 & 1,500 \\
\hline & 2.5 & $\begin{array}{l}\text { Selective Catalytic Reducer } \\
\text { (SCR) }\end{array}$ & & 244,000 & 24,400 & SCR & 43,920 & 4,392 & SCR & 0.0 & 0 \\
\hline & 2.6 & Sub-Totals & & 374,000 & 30,900 & & $37,817,000$ & $1,893,000$ & & $47,237,000$ & $2,362,000$ \\
\hline \multirow[t]{4}{*}{3} & 3.0 & Variable Costs & \multicolumn{3}{|c|}{ Existing System } & \multicolumn{3}{|c|}{ Hybrid System with Thesis FC } & \multicolumn{3}{|c|}{ Hybrid System w/ Commercial FC } \\
\hline & & & & & Annual & & & Annual & & & Annual \\
\hline & & & & & $\$$ & & & $\$$ & & & $\$$ \\
\hline & 3.1 & Cost due to Emissions & & & 52,780 & & & 9,769 & & & 9,769 \\
\hline
\end{tabular}




\begin{tabular}{|c|c|c|c|c|c|c|c|c|c|c|}
\hline & 3.2 & $\mathrm{O} \& \mathrm{M}$ Cost of $\mathrm{GB} / \mathrm{HE}$ & & 26,000 & & & 6,200 & & & 6,200 \\
\hline & 3.3 & $\mathrm{O} \& \mathrm{M}$ Cost of $\mathrm{FC} / \mathrm{HR}$ & & 0 & & & 448,000 & & & 460,000 \\
\hline & 3.4 & Cost of Natural Gas & & 19,414 & & & 3,593 & & & $4,069,000$ \\
\hline & 3.5 & Cost of Hydrogen & & 0 & & & $5,728,000$ & & & 0 \\
\hline & 3.6 & Sub-Totals & & 98,190 & & & $6,196,000$ & & & $4,545,000$ \\
\hline 4 & 4.0 & Additional Incomes & \multicolumn{2}{|l|}{ Existing System } & \multicolumn{3}{|c|}{ Hybrid System with Thesis FC } & \multicolumn{3}{|c|}{ Hybrid System w/ Commercial FC } \\
\hline & & & & Annual & & Annual & Annual & & Annual & Annual \\
\hline & 4.2 & Electric Power (kW-hr) & & - & $\mathrm{FC}$ & $89,530,000$ & $3,859,000$ & $\mathrm{FC}$ & $92,088,000$ & $3,969,000$ \\
\hline & 4.3 & Sub-Totals & & 0 & & & $6,715,000$ & & & $6,825,000$ \\
\hline \multirow[t]{3}{*}{5} & 5.0 & Structural Costs & \multicolumn{2}{|l|}{ Existing System } & \multicolumn{3}{|c|}{ Hybrid System with Thesis FC } & \multicolumn{3}{|c|}{ Hybrid System w/ Commercial FC } \\
\hline & & & Purchase & Annual & & Purchase & Annual & & Purchase & Annual \\
\hline & & & $\$$ & $\$$ & & $\$$ & $\$$ & & $\$$ & $\$$ \\
\hline \multirow{3}{*}{6} & & & & $\$$ & & & $\$$ & & & $\$$ \\
\hline & 6.1 & Total Cost (Annually) & & $1,344,000$ & & & $1,981,000$ & & & 689,000 \\
\hline & 6.2 & $\begin{array}{l}\text { Net Cost Ratio } \\
\text { (Hybrid/Existing) }\end{array}$ & & & & & 1.47 & & & 0.51 \\
\hline
\end{tabular}

\section{Nomenclature:}

GB - Gas burner; FC - Fuel cell; HE - Heat Exchanger; HR - Heat Recouperator; O \& M - Operational and Maintenance Costs; 
- Operating cost of SCR is $\$ 18.75$ per MBtu of gas burner heat produced. Cost due to emissions in existing heating system is $\$ 52,800$ and in hybrid heating system is $\$ 9,800$.

- Cost of natural gas is assumed to $\$ 5$ per MBtu of heat produced and cost of hydrogen is $\$ 7.5$ per MBtu of heat produced.

\subsection{Use of Commercial Fuel Cell in Hybrid Heating System}

A commercial fuel cell is incorporated in hybrid heating system. The fuel cell is developed and manufactured by UTC Power in South Windsor, CT. The fuel cell is called PureCell400 system. PureCel1400 system produces electrical power at 400kW and thermal power of 1.2 MBtu/hr (at 200F).

From the data provided by M-PRT, the maximum thermal power required from fuel cell is calculated as $25 \mathrm{MBtu} / \mathrm{hr}$. Since the fuel cell system is oversized by $25 \%, 27$ PureCell400 Systems are utilized and provide a maximum power of $31 \mathrm{MBtu} / \mathrm{hr}$. Each PureCell400 System produces power at $400 \mathrm{~kW}$. Hence, when 27 PureCell400 systems are connected in series they provide electricity at $10,800 \mathrm{~kW}$ of power.

- Average equipment cost of fuel cell (as provided by UTC power) is $\$ 3500 / \mathrm{kW}$.

- Average installation and preventive maintenance costs for each PureCell400 System is $\$ 400,000$ per PureCell400 (depending on the site they install the fuel cell).

- For 27 PureCell400 systems, the equipment cost could be around $\$ 37,800,000$ and installation cost is about $\$ 10,800,000$.

Hence Capital Cost of 27 PureCell400 Systems (including equipment, installation and preventive maintenance) $=\$ 48,000,000$

Cost assessment for hybrid heating system with commercial fuel cell was also included in the Table 7.1 along with cost assessment for existing and hybrid heating system.

The PureCell400 system comes with a one year warranty at no cost. After that, the warranty can be purchased at $\$ 0.02 / \mathrm{kW}-\mathrm{hr}$ for the full life of fuel cell (which is 20 years). The total power of commercial fuel cell considered for M-PRT application is $10,500 \mathrm{~kW}$. The annual (optional) cost of warranty for commercial fuel cell is $\$ 1,830,000$. 


\subsection{Cost Savings in Hybrid Heating System}

\subsubsection{Savings due to Fuel Cell Generated Electricity and Heat}

Fuel cell operates constantly throughout the year. The remaining fuel cell generated heat shall be sold for commercial purposes. The electrical energy generated from fuel cell can also be sold for commercial use. Selling price of fuel cell electricity is assumed to be 4.3 cents per $\mathrm{kW}-\mathrm{hr}$ for industrial applications [22]. Selling price of fuel cell heat is assumed to be $\$ 12$ per MBtu [23].

The savings from hybrid heating system include the savings due to reduction in emissions. Selective Catalytic Reduction (SCR) process can be used for reducing emissions from natural gas burner.

Total heat required from natural gas burner in existing heating system $=2850 \mathrm{MBtu}$

Total heat required from natural gas burner in hybrid heating system $=520 \mathrm{MBtu}$

Additional heat needed by existing heating system $=(2851-521)=2330 \mathrm{MBtu}$

Cost of reducing emissions using SCR is 64 mills per $\mathrm{kW}-\mathrm{hr}$ or $\$ 19 / \mathrm{MBtu}$

Hence, the additional cost for reducing emissions per year in existing heating system is $\$ 43,700$.

\subsubsection{Structural Savings in Hybrid Heating System}

Hybrid heating system maintains the guideway at constant temperature. This will reduce structural damages to the guideway concrete, pipes etc to a certain extent. Cost for structural replacement in existing heating system can be reduced or may be avoided in hybrid heating system.

- Average cost of construction of M-PRT guideway (in 2004 dollars) is \$8.3 million per lane mile of the guideway.

- BP3 provides heat for the guideway consisting of length 4,390 ft of single lane guideway. Hence, the average cost for BP3 heated guideway is \$36.5 million. 
- The average life of guideway in existing heating system is assumed to be 30 years. Since hybrid heating system maintains the guideway at constant temperature, the guideway may have an average life of 60 years.

\subsubsection{Net Cost Savings in Hybrid Heating System}

Therefore, the total savings in hybrid heating system is the sum of savings due to emissions, savings due to sale of fuel cell generated heat, fuel cell generated electricity and structural savings. The detailed calculations for the amount of savings throughout the year are calculated in Table 7.2. Therefore, the annual savings from hybrid heating system are about $\$ 6,752,000$ (not including the structural savings). The annual structural savings as calculated in Table 7.1 is $\$ 607,000$.

Table 7.2: Savings in Hybrid Heating System

\begin{tabular}{|c|c|c|c|c|}
\hline MONTH & $\begin{array}{c}\text { Savings due } \\
\text { to reduction } \\
\text { in emissions }\end{array}$ & $\begin{array}{c}\text { Savings from } \\
\text { selling fuel } \\
\text { cell } \\
\text { electricity }\end{array}$ & $\begin{array}{c}\text { Savings from } \\
\text { sale of fuel cell } \\
\text { heat }\end{array}$ & $\begin{array}{c}\text { Total Savings } \\
\text { from Hybrid } \\
\text { Heating system }\end{array}$ \\
\hline November & $(\$)$ & $(\$)$ & $(\$)$ & $(\$)$ \\
\hline December & $\$ 2,300$ & $\$ 295,000$ & $\$ 252,000$ & $\$ 547,000$ \\
\hline January & $\$ 37,300$ & $\$ 327,000$ & $\$ 184,000$ & $\$ 534,000$ \\
\hline February & $\$ 1,260$ & $\$ 306,000$ & $\$ 138,000$ & $\$ 502,000$ \\
\hline March & $\$ 2,160$ & $\$ 327,000$ & $\$ 204,000$ & $\$ 450,000$ \\
\hline April & $\$ 0$ & $\$ 316,000$ & $\$ 270,000$ & $\$ 533,000$ \\
\hline May & $\$ 0$ & $\$ 327,000$ & $\$ 279,000$ & $\$ 586,000$ \\
\hline June & $\$ 0$ & $\$ 316,000$ & $\$ 270,000$ & $\$ 586,000$ \\
\hline July & $\$ 0$ & $\$ 327,000$ & $\$ 279,000$ & $\$ 606,000$ \\
\hline August & $\$ 0$ & $\$ 327,000$ & $\$ 279,000$ & $\$ 606,000$ \\
\hline September & $\$ 0$ & $\$ 316,000$ & $\$ 270,000$ & $\$ 586,000$ \\
\hline October & $\$ 0$ & $\$ 327,000$ & $\$ 279,000$ & $\$ 606,000$ \\
\hline TOTAL & $\mathbf{\$ 4 3 , 0 0 0}$ & $\mathbf{\$ 3 , 8 5 8 , 0 0 0}$ & $\mathbf{\$ 2 , 8 5 1 , 0 0 0}$ & $\$ \mathbf{6 6 , 7 5 2 , 0 0 0}$ \\
\hline
\end{tabular}

\subsection{Cost Comparison of Existing and Hybrid Heating Systems}

A cost break-down for existing and hybrid heating systems are calculated in Table 7.3 using fixed costs, variable costs, additional income generated and structural savings. Net 
cost is calculated by subtracting the savings and income generated in heating systems. Net cost ratio is defined as the ratio between the net cost of hybrid heating system and existing heating system.

The net cost of hybrid heating system after subtracting the savings obtained is $\$ 1,989,000$ per year. Therefore the cost of hybrid heating system is more than the cost of existing heating system which is around $\$ 1,363,000$ per year.

The net cost of hybrid heating system using the commercial fuel cell is about $\$ 692,000$ annually which is about $\$ 1,297,000$ lesser than the hybrid heating system using hydrogen fuel cell. This difference is mainly due to usage of natural gas as fuel in commercial fuel cell. Using hydrogen fuel cell in hybrid heating system provides an alternate method of fuelling the M-PRT guideway heating system. The cost ratio of hybrid heating system to existing heating system may reduce by using a lesser size fuel cell. Using a fuel cell producing a maximum power of $15 \mathrm{MBtu} / \mathrm{hr}$ shall make the hybrid heating system have a total cost of $\$ 1,635,000$ and a cost ratio of 1.20.

Table 7.3: Cost Comparison of Existing and Hybrid Heating Systems

\begin{tabular}{|l|c|c|c|c|c|}
\hline $\begin{array}{c}\text { TYPE OF } \\
\text { COSTS }\end{array}$ & $\begin{array}{c}\text { Existing } \\
\text { Heating } \\
\text { System } \\
\text { (\$/year) }\end{array}$ & $\begin{array}{c}\text { Hybrid } \\
\text { Heating } \\
\text { System with } \\
\text { Thesis FC } \\
\text { (with } \\
\text { maxFC } \\
\text { power }=25 \\
\text { MBtu/hr) } \\
\text { (\$/year) }\end{array}$ & $\begin{array}{c}\text { Hybrid } \\
\text { Heating } \\
\text { System with } \\
\text { Thesis FC } \\
\text { (with } \\
\text { maxFCpower } \\
=\mathbf{2 0} \\
\text { MBtu/hr) } \\
\mathbf{( \$ / y e a r ) ~}\end{array}$ & $\begin{array}{c}\text { Hybrid } \\
\text { Heating } \\
\text { System with } \\
\text { Thesis FC } \\
\text { (with } \\
\text { maxFCpower } \\
=\mathbf{1 5} \\
\text { MBtu/hr) } \\
\mathbf{( \$ / y e a r )}\end{array}$ & $\begin{array}{c}\text { Hybrid } \\
\text { Heating } \\
\text { System with } \\
\text { Commercial } \\
\text { FC } \\
\text { (PureCell400 } \\
\text { by UTC } \\
\text { Power) } \\
\text { (\$/year) }\end{array}$ \\
\hline Fixed Costs & 30,900 & $1,894,000$ & $1,518,000$ & $1,146,000$ & $2,360,000$ \\
\hline $\begin{array}{l}\text { Variable } \\
\text { Costs }\end{array}$ & 118,000 & $6,199,000$ & $4,956,000$ & $3,723,000$ & $4,545,000$ \\
\hline $\begin{array}{l}\text { Additional } \\
\text { Income }\end{array}$ & 0 & $6,710,000$ & $5,269,000$ & $3,840,000$ & $6,820,000$ \\
\hline $\begin{array}{l}\text { Structural } \\
\text { Costs }\end{array}$ & $1,215,000$ & 607,000 & 607,000 & 607,000 & 607,000 \\
\hline Net-Cost & $\mathbf{1 , 3 6 3 , 0 0 0}$ & $\mathbf{1 , 9 9 0 , 0 0 0}$ & $\mathbf{1 , 8 1 2 , 0 0 0}$ & $\mathbf{1 , 6 3 5 , 0 0 0}$ & $\mathbf{6 9 3 , 0 0 0}$ \\
\hline Cost Ratio & & $\mathbf{1 . 4 6}$ & $\mathbf{1 . 3 3}$ & $\mathbf{1 . 2 0}$ & $\mathbf{0 . 5 1}$ \\
\hline
\end{tabular}




\subsection{Cost of Hydrogen in Hybrid Heating System}

The cost of hydrogen in hybrid heating system is assumed to be $\$ 7.5$ per MBtu, which is 1.5 times the cost of natural gas. The cost ratio is the ratio of net cost of hybrid heating system to that of existing heating system. The cost ratio decreases with decrease in cost of hydrogen as shown in Figure 7.1. When the cost of hydrogen is $\$ 7.5 / \mathrm{MBtu}$ the net cost ratio reduces to 1 . Hence, the hybrid heating system becomes more cost effective than the existing heating system when the cost of hydrogen drops below \$7.5/MBtu.

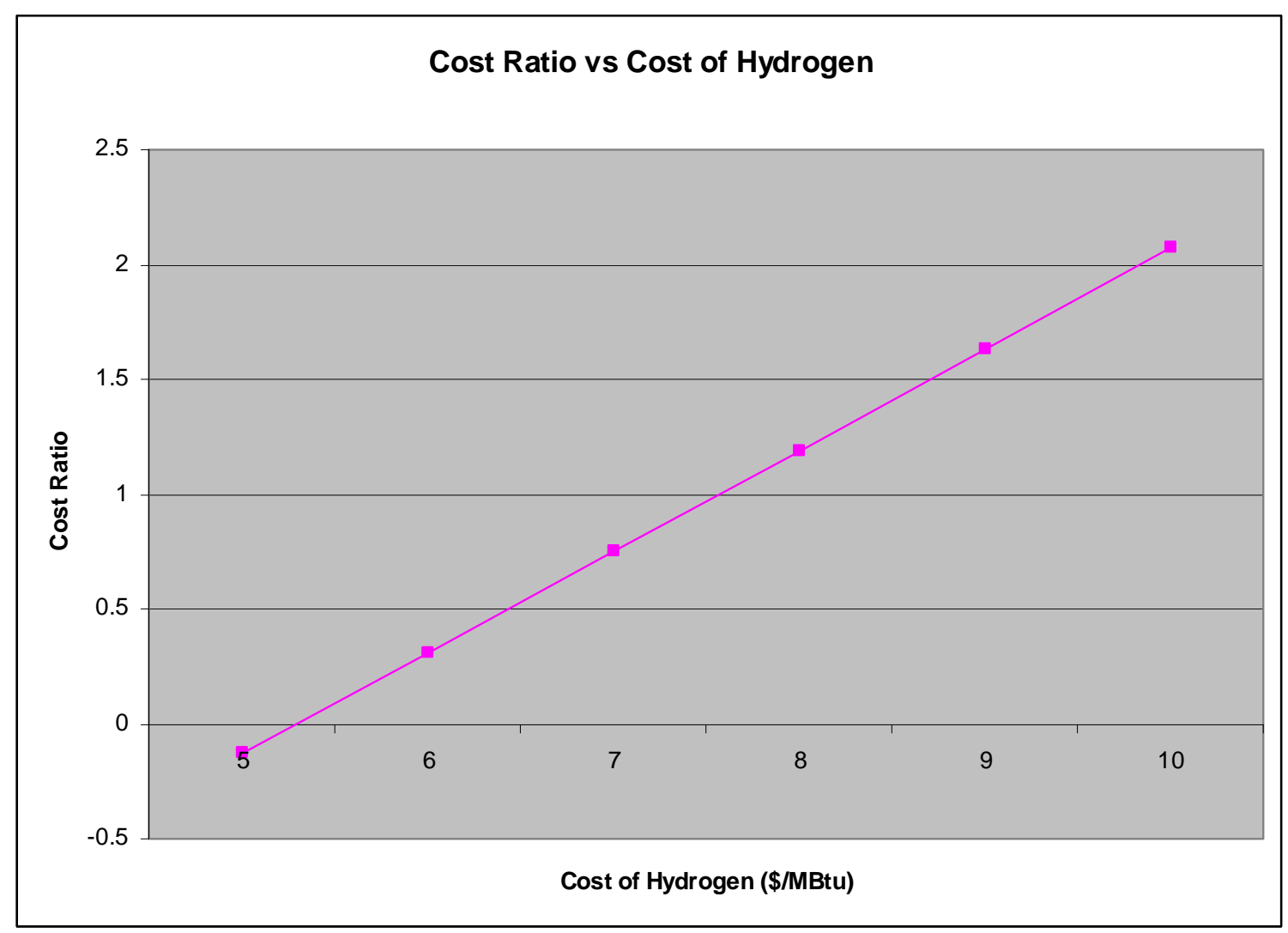

Figure 7.1: Cost Ratios vs. Cost of Hydrogen

The hybrid heating system can have a faster payback when the cost of hydrogen falls below $\$ 7 / \mathrm{MBtu}$. The payback period decreases gradually as the price of hydrogen decreases. 


\subsection{Payback Period for Hybrid Heating System}

The average life of fuel cell is 20 years. The hybrid heating system using hydrogen fuel cell will not recover the capital cost investment during its life time. But the hybrid heating system using commercial fuel cell will recover the capital cost in $75 \%$ of the life of fuel cell.

Hybrid heating system using hydrogen fuel cell:

Total capital cost (in 2008 dollars) of hybrid heating system is $\$ 37.8$ million.

Net annual cost of hybrid heating system (excluding the annual cost of $\mathrm{FC} / \mathrm{HR} / \mathrm{GB} / \mathrm{HE}$ )

$$
=\$ 1,985,481-\$ 1,885,586-\$ 1,250-\$ 300-\$ 1,500=\$ 96,800
$$

where

Net Annual cost of hybrid heating system (using hydrogen fuel cell) is $\$ 1,985,481$ (as shown in Table 7.1 below)

Annual cost of fuel cell (FC) is $\$ 1,885,586$

Annual cost of heat recouperator (HR) is $\$ 1,250$

Annual cost of gas burner (GB) is $\$ 300$

Annual cost of heat exchanger (HE) is $\$ 1,500$

Annual cost of existing heating system (excluding the annual cost of $\mathrm{GB} / \mathrm{HE}$ )

$$
=\$ 1,363,100-\$ 5,000-\$ 2,500=\$ 1,355,600
$$

where

Net Annual cost of existing heating system is $\$ 1,363,100$ (as shown in Table 7.1 below)

Annual cost of gas burner (GB) is $\$ 5,000$

Annual cost of heat exchanger (HE) is $\$ 2,500$

[Note: The costs of $\mathrm{FC} / \mathrm{HR} / \mathrm{GB} / \mathrm{HE}$ are fixed costs. They are excluded from the net annual cost above to estimate the recovery time of capital cost investment.]

Hence the annual cost difference by using hybrid heating system $=\$ 1,259,000$

Payback period $=(\$ 37.8$ million $) /(\$ 1.2$ million $)=31.5$ years

Hybrid heating system using commercial fuel cell: 
Total capital cost (in 2008 dollars) of hybrid heating system is $\$ 47.1$ million.

Net annual cost of hybrid heating system (excluding the annual cost of $\mathrm{FC} / \mathrm{HR} / \mathrm{GB} / \mathrm{HE}$ )

$$
=\$ 694,036-\$ 2,358,804-\$ 1,250-\$ 300-\$ 1,500=-\$ 1,668,000
$$

where

Net Annual cost of hybrid heating system (using hydrogen fuel cell) is $\$ 694,036$ (as shown in Table 7.1 below)

Annual cost of fuel cell (FC) is $\$ 2,358,804$

Annual cost of heat recouperator (HR) is $\$ 1,250$

Annual cost of gas burner (GB) is $\$ 300$

Annual cost of heat exchanger (HE) is $\$ 1,500$

Annual cost of existing heating system (excluding the annual cost of GB/HE)

$$
=\$ 1,363,100-\$ 5,000-\$ 2,500=\$ 1,355,600
$$

where

Net Annual cost of existing heating system is $\$ 1,363,100$ (as shown in Table 7.1 below)

Annual cost of gas burner (GB) is $\$ 5,000$

Annual cost of heat exchanger (HE) is $\$ 2,500$

[Note: The costs of $\mathrm{FC} / \mathrm{HR} / \mathrm{GB} / \mathrm{HE}$ are fixed costs. They are excluded from the net annual cost above to estimate the recovery time of capital cost investment.]

Hence the annual cost difference by using hybrid heating system $=\$ 3,023,000$

Payback period $=(\$ 47.1$ million $) /(\$ 3.02$ million $)=15.6$ years 


\section{Chapter 8}

\section{Results and Discussion}

\subsection{Results from Comparison of Existing and Hybrid Heating Systems}

This thesis designed and analyzed the possibility of a new hybrid heating system for the guideway in the M-PRT. The analysis of the hybrid heating system shows that the total heat to be produced by the natural gas burner and fuel cell are 521 MBtu and 37,338 MBtu respectively.

\subsubsection{Hybrid Heating System using Hydrogen FC}

The total cost of fuel required is $\$ 5,730,000$. The total savings from hybrid heating system is about $\$ 6,720,000$ which includes savings due to reduction in emissions, savings from selling fuel cell electricity and heat and structural savings.

Hence, the net annual expenditure for hybrid heating system after deducting the savings is about $\$ 1,985,000$ and the net expenditure in existing heating system is $\$ 1,344,000$. The capital cost of fuel cell is calculated to be around $\$ 37,712,000$. Hence, the hybrid heating system shall have a 31 year payback period for the capital cost incurred. These estimates are based on a specific price for natural gas and hydrogen. If the cost of hydrogen decreases, the hybrid heating system can have faster payback as shown in Figure 7.1.

\subsubsection{Hybrid Heating System using Commercial FC}

The cost comparison of hybrid heating system using commercial FC for varying power of fuel cell is shown in Table 8.1 below. The cost of hybrid heating system decreases by increasing the size of fuel cell. When using a commercial FC with natural gas as fuel the hybrid heating system is more efficient at higher size. By reducing the size of fuel cell the fixed and variable costs decrease. But the additional income 
generated by fuel cell decreases at a higher rate with reduction in size. Hence, the MPRT can choose the fuel cell that can supply the entire convection heat required by the guideway.

Table 8.1: Comparison of Hybrid Heating System for varying power from commercial $\mathrm{FC}$

\begin{tabular}{|l|r|r|r|r|}
\hline & \multicolumn{4}{|c|}{$\begin{array}{l}\text { Hybrid heating system when maximum } \\
\text { power (in MBtu/hr) of commercial FC is }\end{array}$} \\
\hline & $\mathbf{3} 30$ & $\mathbf{2 5 5}$ & $\mathbf{2} \mathbf{2 0}$ & $\mathbf{2 1 5}$ \\
\hline Fixed Costs & $2,828,000$ & $2,360,000$ & $1,886,000$ & $1,415,000$ \\
\hline Variable Costs & $5,442,000$ & $4,545,000$ & $3,634,000$ & $2,730,000$ \\
\hline Additional incomes & $8,260,000$ & $6,820,000$ & $5,357,000$ & $3,906,000$ \\
\hline Structural Savings & 607,000 & 607,000 & 607,000 & 607,000 \\
\hline Net Cost & $\mathbf{6 1 6 , 0 0 0}$ & $\mathbf{6 9 3 , 0 0 0}$ & $\mathbf{7 7 0 , 0 0 0}$ & $\mathbf{8 4 6 , 0 0 0}$ \\
\hline Cost Ratio & $\mathbf{0 . 4 6}$ & $\mathbf{0 . 5 2}$ & $\mathbf{0 . 5 7}$ & $\mathbf{0 . 6 3}$ \\
\hline
\end{tabular}

The hybrid heating system requires a maximum power of $25 \mathrm{MBtu} / \mathrm{hr}$ to maintain the guideway at $43^{\circ} \mathrm{F}$. Hence, the hybrid heating system consisting of commercial $\mathrm{FC}$ with a maximum power of $25 \mathrm{MBtu} / \mathrm{hr}$ may be the best alternative for supplying heat to the MPRT guideway. A cost comparison chart is shown in Figure 8.1.

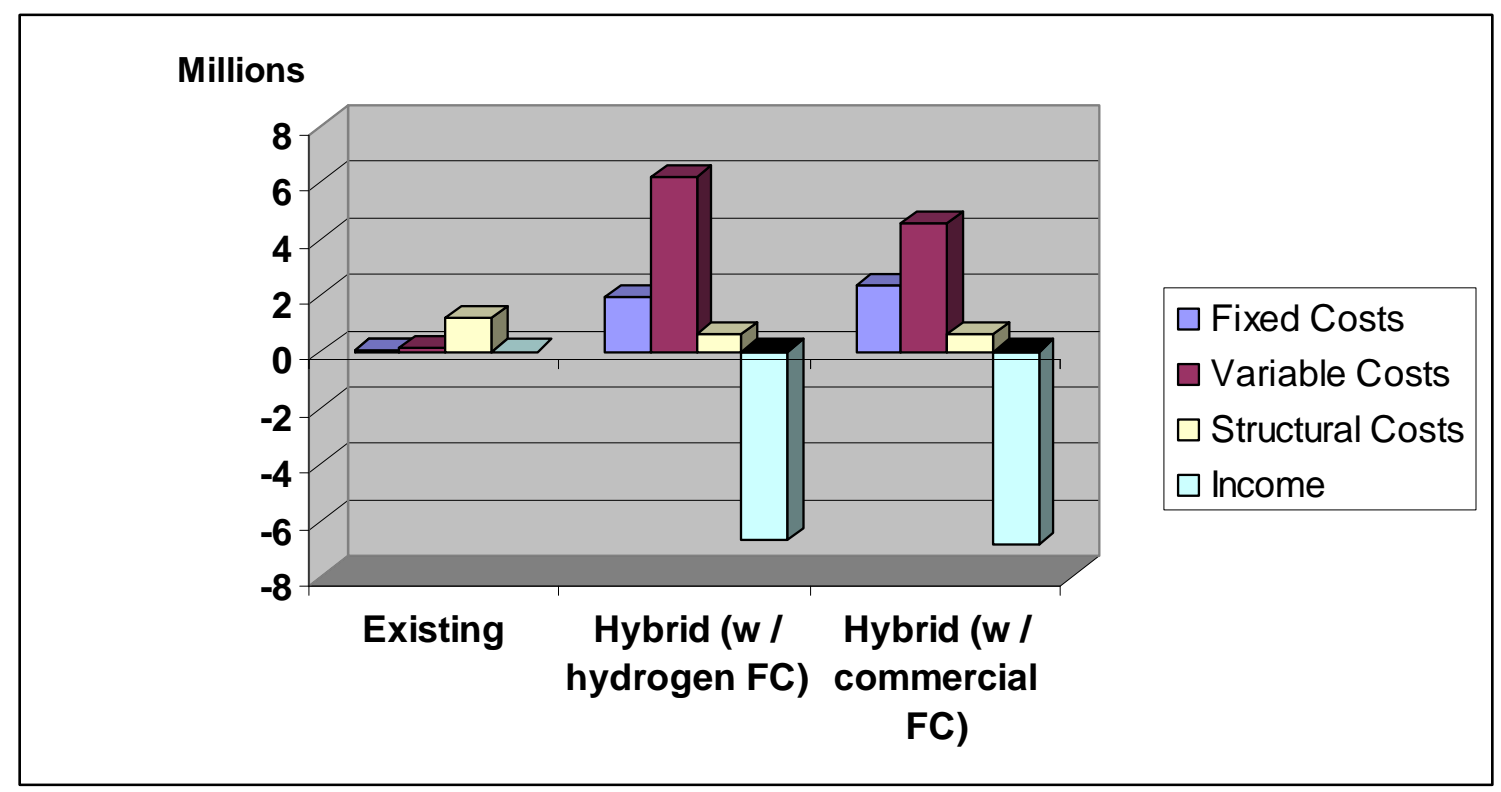

Figure 8.1: Cost Comparison of Existing and Hybrid Heating Systems

where

Fixed costs - capital costs

Variable costs - utility costs, emissions reduction costs and $\mathrm{O} \& \mathrm{M}$ costs 
Structural costs - structural maintenance costs

Income - obtained from sale of electricity and sale of available heat

The net annual savings for hybrid heating system excluding the cost of commercial FC is $\$ 1,665,000$. In comparison to the existing heating system, the hybrid heating system is $\$ 3$ million less expensive every year. Hence, the hybrid heating system can recover the capital investment in 16 years.

\subsection{Conclusions}

The proposed hybrid heating system that adds a commercial fuel cell (PureCell400 system) in parallel with the gas burner is recommended for implementation at M-PRT to improve the guideway heating system. Such a hybrid heating system is $48 \%$ more cost effective, produces $82 \%$ less emissions and is $50 \%$ more reliable when compared to the existing heating system as shown in Figure 8.2 and Figure 8.3.

There are several advantages by implementing a hybrid guideway heating system for M-PRT as mentioned below. The income obtained from sale of electricity and available heat, the reduction in environmental emissions and the improved system reliability highly contribute towards a $48 \%$ lower net annual cost for the hybrid heating system when compared with existing heating system.

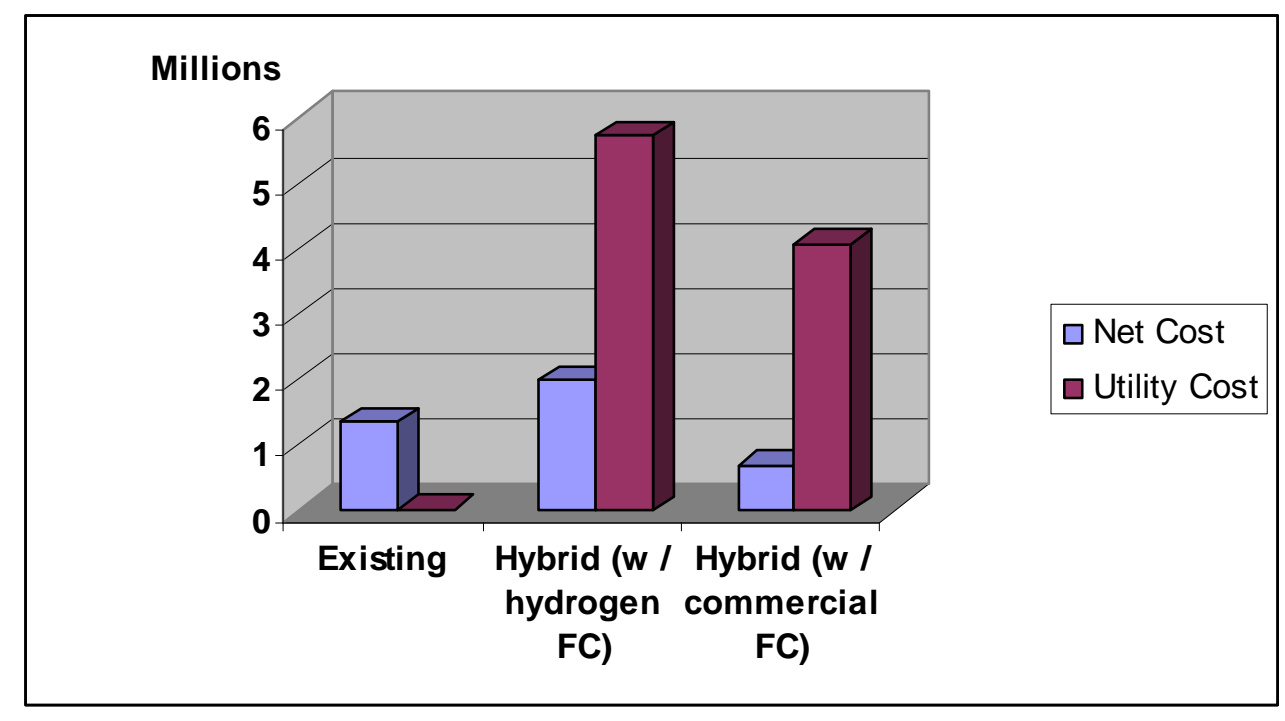

Figure 8.2: Net and Utility Cost Comparison 
A commercial fuel cell system has a $25 \%$ higher capital cost than hydrogen fuel cell. But the commercial fuel cell using natural gas as fuel has a $29 \%$ lower utility costs when compared to hydrogen fuel cell using hydrogen. Therefore, the hybrid heating system using a commercial fuel cell (PureCell400 system) recovers the capital cost 51\% faster than the hybrid heating system using hydrogen fuel cell.

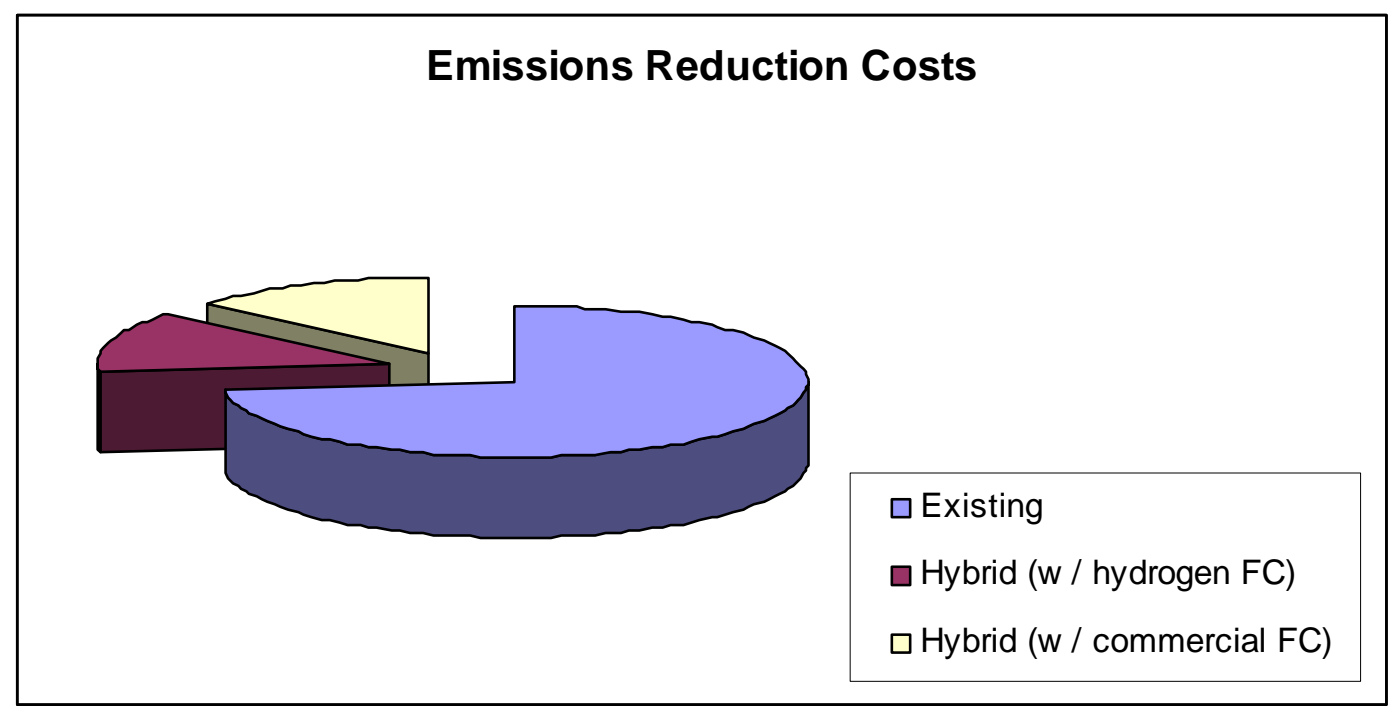

Figure 8.3: Reduction in Emissions

Hence, the hybrid heating system using commercial fuel cell is recommended for implementation at M-PRT to improve the existing guideway heating system. 


\section{Chapter 9}

\section{Contributions and Accomplishments}

\subsection{Contributions and Accomplishments}

The primary contribution of this thesis was to show that a fuel cell could be used as a second source of heat for M-PRT guideway heating system that would be more costeffective to operate and would produce lower emissions than the existing guideway heating system. Such a hybrid heating system shall be designed so that the fuel cell produced heat will be used to maintain the M-PRT guideway at an operating temperature of $43^{\circ} \mathrm{F}$ throughout the winter. The existing natural gas heater in hybrid heating system will provide heat to melt all precipitated snow. A commercial fuel cell will be recommended as it has lower utility cost than hydrogen fuel cell. The utility costs of existing heating system are lowered by using hybrid heating system.

The primary accomplishment of this thesis was the design of a hybrid heating system that added a fuel cell of electrical power $10,220 \mathrm{~kW}$ to the existing gas burner so that the combined hybrid heating system is more cost effective than the existing guideway heating system. The hybrid heating system is a Combined Heat and Power (CHP) system that supplies $13.5 \%$ of the heat that it generates to the M-PRT and provides $10.2 \mathrm{MW}$ of power to the local grid. It generates $\$ 3,853,000$ annual income through the sale of electricity and $\$ 2,846,000$ annual income through the sale of available heat. The hybrid heating system also annually saves $\$ 607,000$ in maintenance costs by increasing the life of M-PRT guideway structure. The hybrid heating system produces lower emissions and thereby reducing the cost of emissions reductions through SCR by $\$ 43,000$ annually. A commercially available PureCell400 fuel cell (produced by UTC power) was selected for incorporation into the hybrid heating system. The hybrid heating system (using commercial fuel cell) proved to be $48 \%$ more cost-effective to operate annually for 20 years than the existing guideway heating system. The utility cost of commercial fuel cell is $28 \%$ lower than that of hydrogen fuel cell and provides $\$ 110,000$ more savings from the sale of electricity. 


\subsection{Recommendations for Future Work}

1. The fuel cell system is being used as a source of additional heat. But the fuel cell used in hybrid heater is a combined heat and power source (Refer to Appendix A). A further study can be performed for an effective usage of the electricity produced to increase the efficiency of the hybrid system. That will help in reducing the payback period.

2. The analysis of the hybrid heating system can be extended for the entire guideway of the M-PRT.

3. Detailed modeling of the entire system can effectively improve the design of hybrid guideway heating system. It can also help in identifying a suitable location for installing the fuel cell unit.

4. A more detailed cost analysis of the system could be helpful to proceed towards installation.

5. Consideration can be given to operating the hybrid heating system in a demand response mode where the fuel cell maintains the guideway at a reduced $35^{\circ} \mathrm{F}$ during non-snowing conditions. Thus, the power requirement of the fuel cell might be reduced significantly without any reduced structural concern.

6. A cost comparison for hybrid heating system can be done by reducing the size of fuel cell to around 15MW and supplementing the fuel cell heat with additional heat from the natural gas burner.

7. A further conversation with UTC power is recommended to confirm how the PureCell400 system manages the $\mathrm{CO}_{2}$ emissions. 


\section{REFERENCES}

1. James Larminie, Andrew Dicks, Fuel Cell Systems Explained, John Wiley \& Sons, Inc., 2002, p 15, 206-209

2. A. F. Massardo, F. Lubelli, Internal Reforming Solid Oxide Fuel Cell-Gas Turbine Combined Cycles (IRSOFC-GT): Part A - Cell Model and Cycle Thermodynamic Analysis, Journal of Engineering for Gas Turbines and Power, 122,2000, p $27-35$

3. S. Campanari, Thermodynamic model and parametric analysis of a tubular SOFC module, Journal of Power Sources, Volume 92, Issues 1-2, 2001, p 26 - 34

4. J. Padulles, G.W. Ault, J.R. McDonald, An integrated SOFC plant dynamic model for power systems simulation, Journal of Power Sources, Volume 86, Issues 1-2, 2000, p 495 - 500

5. Zhixin Miao, Modeling and Dynamic Stability of Distributed Generation, Ph.D. Dissertation, West Virginia University, 2002

6. R. H. Lasseter, Control of Distributed Resources, Proceedings of the 1998 International Conference on Bulk Power Systems Dynamics and Control IV Restructuring, Santorini, Greece, August 1998, pp. 323-330

7. Padmanabhan Srinivasan, Dynamic Modeling and Control of a Proton Exchange Membrane Fuel Cell as a Distributed Generator, West Virginia University, 2003

8. Praveen K. Kuntamalla, John E. Sneckenberger, Report on Hybrid Heating System for M-PRT Project, Advance Power Electricity Research Center (APERC)

9. "Energy Information Administration reports", U. S. Department of Energy, Washington, DC, December 2003

URL: http://www.eia.doe.gov/calendar/reports.htm http://www.eia.doe.gov/oiaf/aeo/gas.html

10. Ambitrol Inhibited Glycol-Based Coolants, Engineering and Operating Guide, DOW Chemical Company, 1991, pp 45-52

11. S. H. Chan, K. A. Khor, Z. T. Xia, A complete polarization model of a solid oxide fuel cell and its sensitivity to the change of cell component thickness, Journal of power sources 93, 2001, pp 130-140 
12. Kenneth K. Humphreys, Jelen's Cost and Optimization Engineering, third edition, McGraw Hill, Inc., 1991, pp 434-456

13. Woodruff, M.G. ; Roop, J.M. ; Seely, H.E.; Jones, T.W.; Dowd, J., Analysis of Energy-Efficiency Investment Decisions by Small and Medium-Sized Manufacturers, U.S. Department of Energy Office of Policy and Office of Energy Efficiency and Renewable Energy, 1996

14. James Larminie, Andrew Dicks, Fuel Cell Systems Explained, John Wiley \& Sons, Inc., 2002, pp 215-226, 229, 232-250, 279-304

15. Anthony F. Mills, Basic Heat and Mass Transfer, Irwin Publishers, Inc., 1995, pp 8-29

16. Valery Knyazkin, Lennart Soder, Claudio Canizares, Control Challenges of Fuel Cell-Driven Distributed Generation, IEEE Bologna Power Tech, Bologna Italy, 2003

17. Stephenson, D., and Ritchey, I., Parametric Study of Fuel Cell Gas Turbine Combined Cycle Performance, 1997, ASME Paper 97-GT-340

18. Drenckhahn, W., Lezuo, A., Fuel Cells for Decentralized Cogeneration Plants, 1996, Power Gen Europe, Budapest

19. Hirano, A., Suzuki, M., Ippommatsu, M., Evaluation of New Solid Oxide Fuel Cell System by Non-Isothermal Modeling, Journal for Electrochemical Society, Vol. 139, Issue 10, 1992

20. Ambitrol Inhibited Glycol-Based Coolants, Engineering and Operating Guide, DOW Chemical Company, 1991, pp 2-4

21. Cost and Performance Baseline for Fossil Energy Plants, Vol. 1, DOE/NETL2007/1281, May 2007, pp 1-3

22. Energy Information Administration, Form EIA-826, "Monthly Electric Sales and Revenue Report with State Distributions Report", Oct 2009.

URL: http://www.eia.doe.gov/cneaf/electricity/epm/table5_6 a.html

23. Energy Information Administration, Form EIA-826, "Consumption, Prices and Expenditures through 2006 for Heat", Oct 2009.

URL: http://www.eia.doe.gov/emeu/states/_seds.html 
24. US DoT RBT Abstract for Contract FTA-WV-26-7001, "Evaluation of Fuel Cell and ITS Technology Applications for Morgantown PRT", Sep 2002.

URL: http://rip.trb.org/browse/printview.asp?ids=6895

25. USDoT/FTA Phase 1 - Report for Contract FTA-WV-26-7001, "Morgantown Personal Rapid Transit System - Energy Assessment Project (Phase I)", July 2004. 


\section{APPENDIX A}

\section{Fuel Cell as Combined Heat and Power Source}

\section{A.1 Introduction}

Combined Heat and Power (CHP) is a very efficient technology for generating electricity and heat together.

CHP is an installation where there is a simultaneous generation of usable heat and power (usually electricity) in a single process. The term CHP is synonymous with 'cogeneration' and 'total energy'. A CHP plant might comprise one or more prime movers usually driving electrical generators, where the heat generated in the process is utilized via suitable heat recovery equipment for a variety of purposes including industrial processes, community heating, space heating and as a replacement to the conventional heaters.

CHP has a better efficiency by generating electricity and heat simultaneously. By utilizing the heat from electricity generation and avoiding transmission losses as electricity is generated on site, CHP typically achieves a 35 percent reduction [18] in primary energy usage compared with power stations and conventional boilers. This can allow the host organization to make economic savings where there is a suitable balance between the heat and power loads. The current mix of CHP installations achieves a reduction of over 30 percent in $\mathrm{CO}_{2}$ emissions in comparison with generation from coalfired power stations, and over 10 percent in comparison with gas fired combined cycle gas turbines. The newest installations achieve a reduction of over 50 percent compared with generation from coal-fired power stations. The Figure A.1 shows why a CHP is preferred in comparison to the conventional power generation. The waste energy in conventional power generation is more when compared with the CHP application. During electricity generation, a large amount of low-grade heat is produced as a byproduct. In conventional power stations this heat is lost. In combined heat and power (CHP) systems, the heat produced during electricity generation is recycled rather than 
wasted, thereby increasing the efficiency of the system. CHP is usually only used as a supplement to grid mains electricity supply.
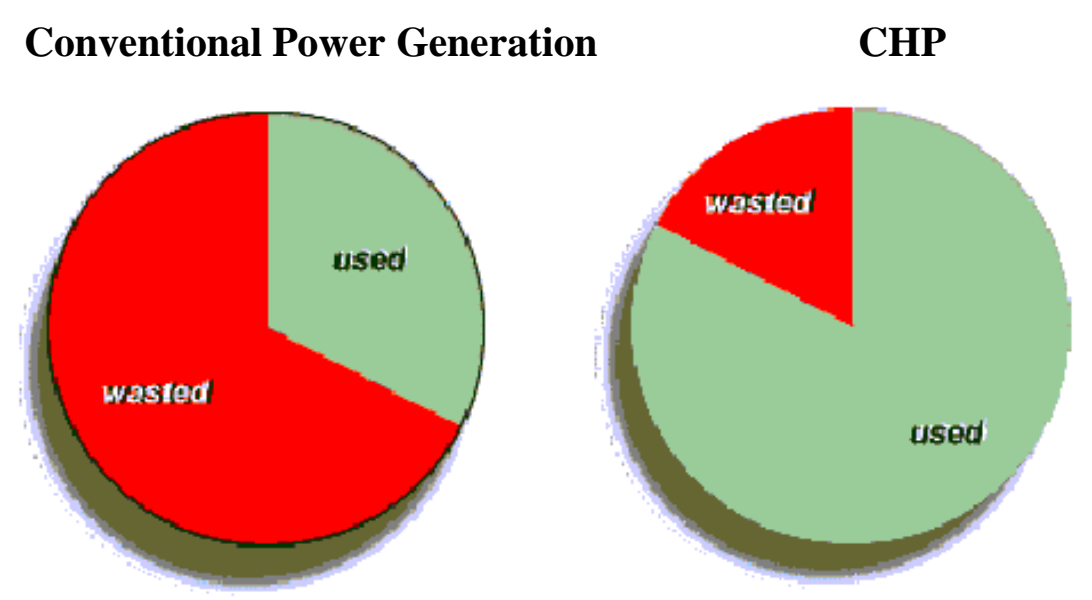

Figure A.1: Conventional Power Generation and Combined Heat and Power (CHP)

High capital and maintenance costs deter individual users from investing in CHP, and therefore CHP schemes are more likely to be used by the public, industrial and commercial sector. The main markets tend to be those requiring a great deal of heat, for example leisure centers, hospitals and industrial sites with process heating requirements, especially the chemical, brewing and paper industries. Sewage treatment works sometimes use CHP fuelled by biogas, emissions released during the decomposition of sewage.

\section{A.2 Fuel Cell as a Combined Heat and Power (CHP) Source}

The energy available in nature takes different forms. The inherent energy in a particular form can be utilized perfectly only if there is a proper energy conversion. Fuel cell is a modern method of energy conversion with lesser pollution, better efficiency and flexible fuel utilization. The chemical energy of hydrogen and oxygen is being converted to thermal and electrical energy simultaneously.

In fuel cell the conversion energy is a direct electrochemical kinetic process and it is not subjected to the limitations of the Carnot cycle. They, as alternatives to conventional energy conversion systems, have the prospect for exploiting fossil fuels more benignly 
and efficiently. The energy losses occurring in the fuel oxidation process is small as compared to the conventional combustion. The electrochemical oxidation of the fuel is accompanied by the release of heat energy and electron flow.

Using a fuel cell only on power generation or heat generation the energy conversion rate is very low. Depending on the type of the fuel cell for a specified input fuel, the electrical energy produced would range from $45 \%$ to $70 \%$ and the thermal energy produced would be in the range of $20 \%$ to $40 \%$. For high temperature fuel cells, the thermal energy produced would be promisingly high. So when the fuel cell is considered for a unique purpose of generating electricity the efficiency of the system would come down. Hence, considering fuel cell as a combined heat and power source would improve the efficiency to a considerable extent. 


\section{APPENDIX B}

\section{Heat Produced from Solid Oxide Fuel Cell}

\section{B.1 Solid Oxide Fuel Cell:}

Solid Oxide Fuel Cell (SOFC) is a high temperature fuel cell considered for this application in hybrid heating system. The schematic of the SOFC is shown in Figure B.1.

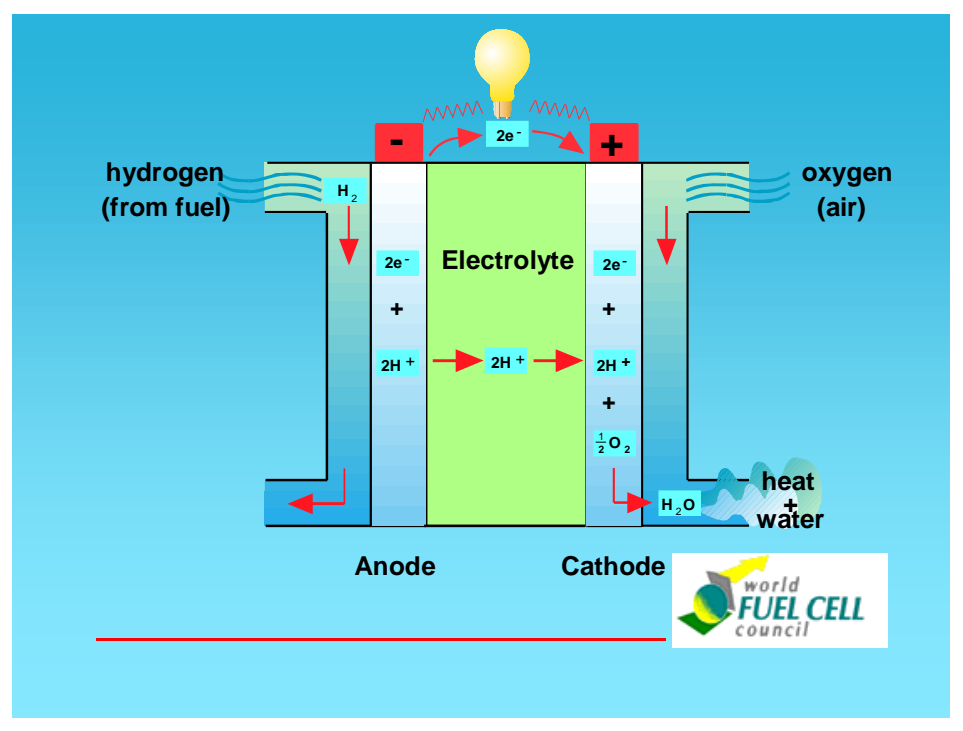

Figure B.1: Solid Oxide Fuel Cell

\section{B.2 Chemical Equations Associated with SOFC:}

SOFC can be fuelled, either by using the natural gas as the fuel or by using the hydrogen directly. The natural gas, if used, breaks down into hydrogen by undergoing a reforming process. The chemical equations associated with the reforming and generation of electricity as follows:

Reforming:

$\mathrm{CH} 4+\mathrm{H} 2 \mathrm{O} \rightarrow \mathrm{CO}+3 \mathrm{H} 2$

Water - Shift Reaction: (Endothermic Reaction) 
Electrochemical Reaction:

There are two kinds of reforming that can take place in an SOFC. They are (i) Internal Reforming SOFC \& (ii) External Reforming SOFC. In an internal reforming SOFC, the natural gas reforms into hydrogen inside the fuel cell and thereby reducing the expense of an external reformer. The external reformer also requires additional cooling for the SOFC stack. Hence, to obtain the suitable heat for the hybrid heating system from the fuel cell, the internal reforming process would be more efficient.

\section{B.3 Equations for Heat Produced in the Reforming and Shifting Reactions:}

For the above equations, the molar flow rates of the inlet gases can be obtained theoretically by using the Newton-Raphson method of solving the non-linear equations. Here $\mathrm{x}, \mathrm{y}$ and $\mathrm{z}$ are the molar flow rates of the gases (methane, carbon monoxide and hydrogen) in the above equation of SOFC. The total heat obtained from the process can be calculated by knowing the enthalpies of the gases mentioned. The reforming and shifting reactions are endothermic. They absorb heat for the reaction to proceed. The electrochemical reaction is exothermic and it releases high amount of heat during the process.

The heat absorbed in the reforming and shifting reaction is obtained from

$Q_{r}=x\left(h_{C O}+3 h_{H_{2}}-h_{\mathrm{H}_{2} \mathrm{O}}-h_{\mathrm{CH}_{4}}\right)$

$Q_{s}=y\left(h_{\mathrm{CO}_{2}}+h_{\mathrm{H}_{2}}-h_{\mathrm{CO}}-h_{\mathrm{H}_{2} \mathrm{O}}\right)$

Where, $\mathrm{x}$ and $\mathrm{y}$ are the molar flow rates,

$h$ is the enthalpy of the gas,

Qr and Qs are the heat absorbed in the reforming and shifting reactions respectively. 


\section{B.4 Characteristics of SOFC:}

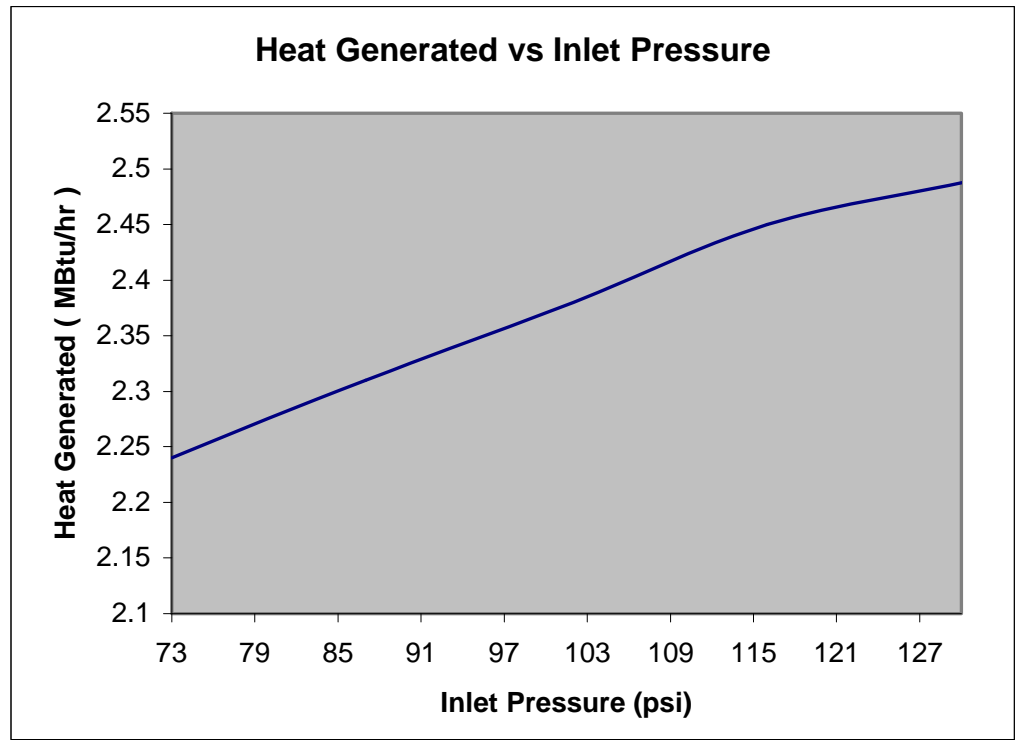

Figure B.2: Heat Generated vs Inlet Pressure

Varying the inlet pressure of the fuel can control the heat produced from the SOFC. Increasing the inlet pressure increases the heat generated and also the operating temperature of the fuel cell as shown in Figures B.2 and B.3. The guideway heating system needs to be operated only during snowing conditions.

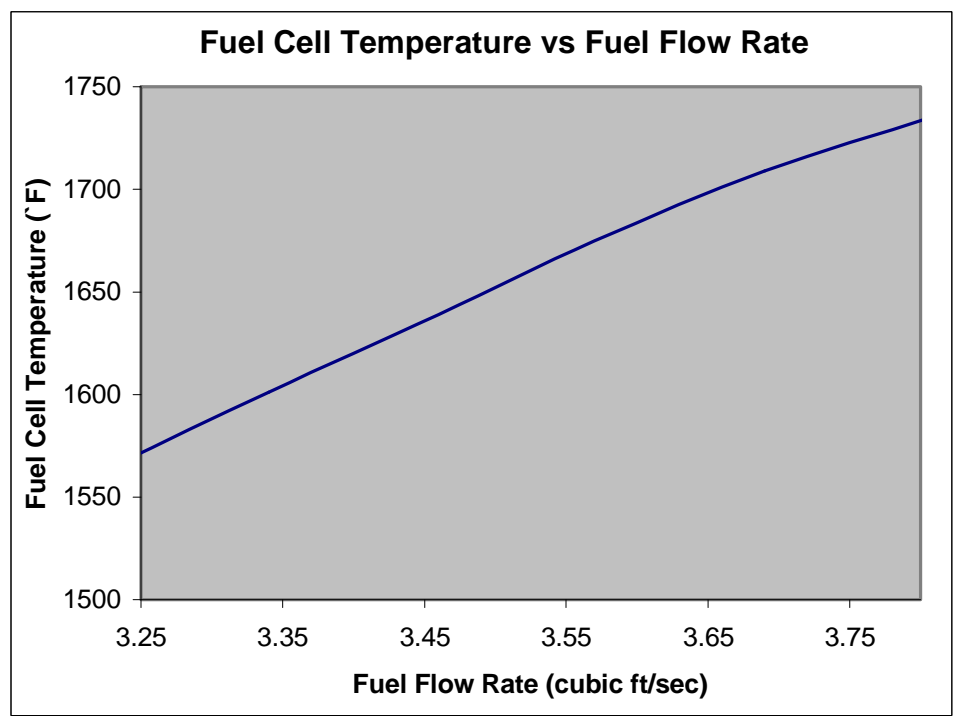

Figure B.3: Fuel Cell Temperature vs Fuel Flow Rate 
Hence, the inlet pressure of the gas is varied accordingly to obtain higher operating temperatures and higher heat requirements. The increase in the operating temperature implicitly means that there is also an increase in the exit flow temperature of the cell. The total heat available from the fuel cell is small in comparison with the heat required for the guideway in the M-PRT. Hence, the SOFC can be used as a supplemental heat source for the existing heating system. The SOFC provides a part of the heat required for the heating system. The percentage of heat it produces depends on the ambient temperature of the proposed hybrid heating system. 


\section{APPENDIX C}

\section{Fuel Cell as an Auxiliary Power Unit in Sleeper Trucks}

The purpose of this Appendix is to provide an overview of the project pursued by the author of this thesis during the period of his practical training employment with International Truck \& Engines.

Solid Oxide Fuel Cell (SOFC) is a potential source of useful thermal and electrical energy for certain applications. This combined heat and power (CHP) feature of the SOFC encouraged International Truck \& Engines (ITE) to pursue a project to develop a prototype of the SOFC as a component of an Auxiliary Power Unit (APU) for use in sleeper trucks.

This SOFC APU project supports the elimination of truck engine idling during waiting periods and regulated rest periods, and thus could significantly reduce overall fuel consumption and improve ambient air quality by reducing emissions. A SOFC APU offers lower fuel consumption, emissions, noise and vibration than a conventional APU. A SOFC APU has the potential to provide thermal heat during the winter and electrical power during the summer to the heating, ventilation and air-conditioning (HVAC) system for the truck. To minimize the need for multiple fuels in the truck, the SOFC developed for this project will be diesel fuelled.

On an average, a truck idles for eight hours a day. Hence, these APUs supply continuous power for a minimum of eight hours. This project first identified all the design parameters for modeling the fuel cell. An analysis was then performed to determine the different thermal and electrical load requirements in the truck during engine idling and engine-off operations, with peak and average load requirements estimated during different climatic conditions. A MATLAB model, as shown in Figure C.1, was developed and simulations are performed for the SOFC APU system, which

include the fuel cell, battery pack, inverter and alternator for the various thermal as well as DC and AC electrical loads. The results of these simulations provided a basis for 
sizing the SOFC APU that can support the truck's HVAC system during engine idling and engine-off operations.

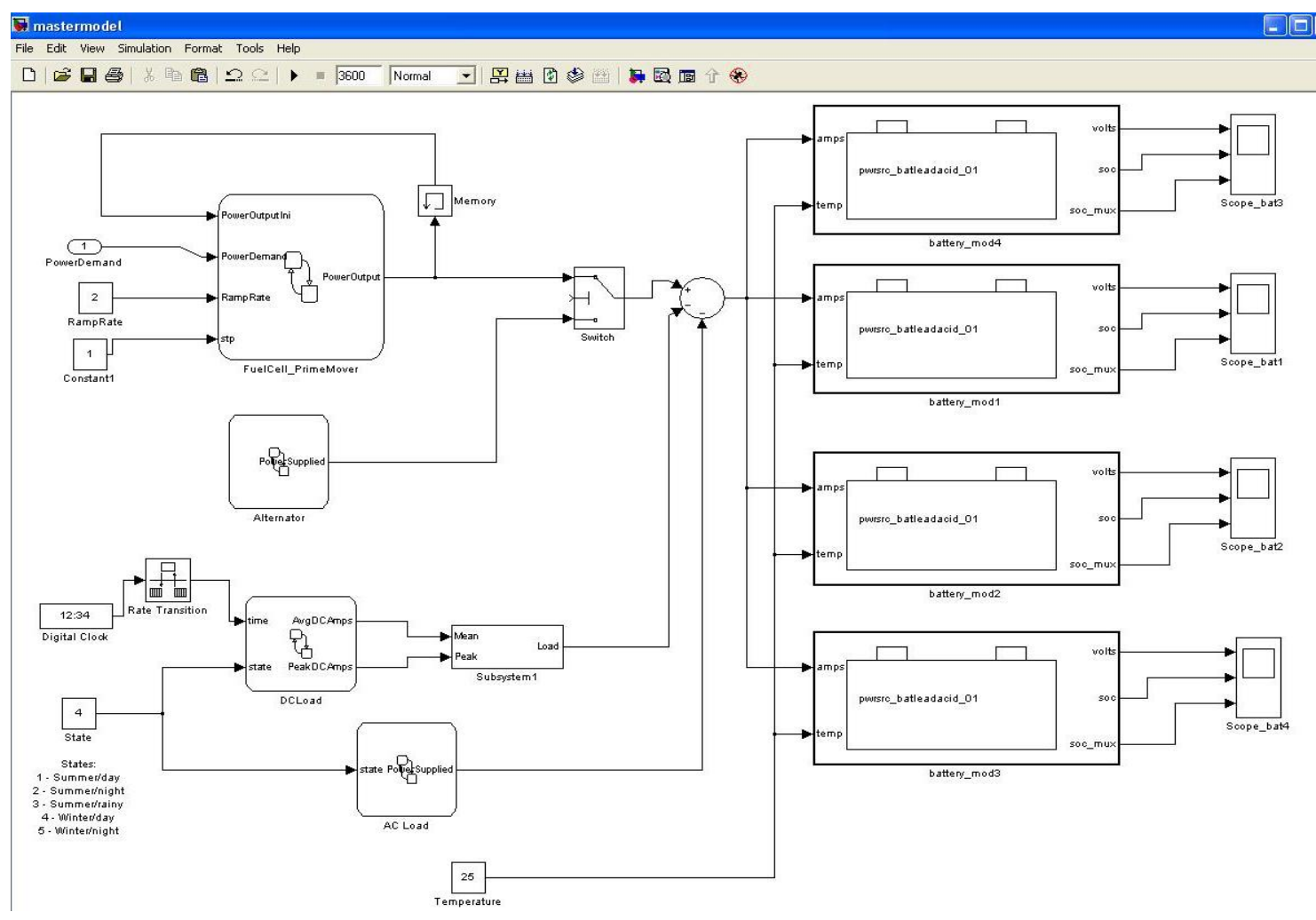

Figure C.1: SOFC Auxiliary Power Unit System Model

The six major contributions in this project were

1. Identified the different electrical and thermal loads for an idling truck.

2. Estimated the peak and average loads for an idling truck.

3. Performed modeling and simulation to size the SOFC for an engine-off truck and to design the power electronics required to effectively operate the fuel cell.

4. Provided requirements to develop and size a Catalytic Partial Oxidation (CPOX) reformer to reform diesel fuel to hydrogen fuel.

5. Packaged the properly sized SOFC APU and found an appropriate location on the truck for the SOFC APU to supply the required electrical and thermal loads during engine-off conditions.

6. Tested the SOFC APU for its mechanical design, vibration behavior and energy efficiency during truck operation and engine-off condition. 


\section{Appendix D}

\section{Data Provided by M-PRT}

M-PRT has provided us with some real-time data which includes the date, time on, time off, average ambient temperature and average rate of snowfall for the period of December 2003 and March 2004 as shown in Table D.1. The time on and time off represent the start time and end time of boiler plant operation on each day during winter. The difference in time on and time off is the hours of operation of gas burner every day. It is also assumed to be the hours of snowfall every day as the gas burner is operated only when it snows.

Table D.1: Data Provided by M-PRT

\begin{tabular}{|c|c|c|c|c|}
\hline Date & Time ON & Time OFF & $\begin{array}{c}\text { Average } \\
\text { Ambient } \\
\text { Temperature }\end{array}$ & $\begin{array}{c}\text { Average } \\
\text { Snowfall Rate }\end{array}$ \\
\hline (MM/DD/YY) & (HH:MM) & (HH:MM) & $\left({ }^{\circ} \mathrm{F}\right)$ & (in./hr) \\
\hline $11 / 29 / 03$ & $5: 20$ & $10: 32$ & 29 & 0.47 \\
\hline $12 / 05 / 03$ & $6: 31$ & $8: 30$ & 36 & 0.36 \\
\hline $12 / 05 / 03$ & $14: 28$ & $17: 51$ & 35 & 0.39 \\
\hline $12 / 05 / 03$ & $20: 36$ & $21: 53$ & 35 & 0.39 \\
\hline $12 / 06 / 03$ & $1: 17$ & $3: 40$ & 34 & 0.40 \\
\hline $12 / 06 / 03$ & $6: 50$ & $8: 00$ & 28 & 0.48 \\
\hline 01/05/04 & 9:43 & $12: 03$ & 28 & 0.48 \\
\hline 01/05/04 & $15: 35$ & $17: 14$ & 28 & 0.48 \\
\hline $01 / 06 / 04$ & $1: 27$ & $4: 02$ & 33 & 0.40 \\
\hline 01/06/04 & $7: 17$ & $9: 48$ & 31 & 0.42 \\
\hline $01 / 07 / 04$ & $6: 56$ & $11: 34$ & 10 & 0.90 \\
\hline $01 / 08 / 04$ & $15: 29$ & 19:05 & 30 & 0.41 \\
\hline $01 / 08 / 04$ & $21: 43$ & $1: 47$ & 27 & 0.47 \\
\hline $01 / 09 / 04$ & $4: 48$ & $5: 38$ & 27 & 0.46 \\
\hline $01 / 10 / 04$ & $8: 40$ & $12: 25$ & 10 & 0.90 \\
\hline $01 / 13 / 04$ & 11:05 & $12: 50$ & 37 & 0.35 \\
\hline $01 / 13 / 04$ & $15: 31$ & $18: 21$ & 32 & 0.41 \\
\hline $01 / 14 / 04$ & $2: 06$ & $3: 49$ & 26 & 0.46 \\
\hline $01 / 14 / 04$ & $17: 34$ & $19: 57$ & 35 & 0.39 \\
\hline $01 / 15 / 04$ & $3: 00$ & $5: 27$ & 38 & 0.34 \\
\hline $01 / 15 / 04$ & $6: 36$ & $8: 06$ & 28 & 0.48 \\
\hline $01 / 15 / 04$ & $10: 13$ & $11: 31$ & 27 & 0.34 \\
\hline $01 / 17 / 04$ & $8: 25$ & $15: 05$ & 16 & 0.47 \\
\hline $01 / 20 / 04$ & $8: 16$ & 9:54 & 19 & 0.85 \\
\hline $01 / 20 / 04$ & $21: 50$ & $5: 04$ & 18 & 0.85 \\
\hline $01 / 22 / 04$ & $20: 27$ & $22: 00$ & 25 & 0.76 \\
\hline
\end{tabular}




\begin{tabular}{|c|c|c|c|c|}
\hline $01 / 22 / 04$ & $22: 11$ & $1: 38$ & 25 & 0.80 \\
\hline $01 / 22 / 04$ & $6: 13$ & 9:07 & 25 & 0.44 \\
\hline $01 / 23 / 04$ & $2: 49$ & $5: 19$ & 12 & 0.43 \\
\hline $01 / 23 / 04$ & $18: 08$ & $23: 59$ & 18 & 0.44 \\
\hline $01 / 23 / 04$ & $12: 58$ & $15: 07$ & 18 & 0.86 \\
\hline $01 / 24 / 04$ & $6: 12$ & $12: 03$ & 21 & 0.80 \\
\hline $01 / 24 / 04$ & 3:08 & $4: 43$ & 20 & 0.80 \\
\hline $01 / 25 / 04$ & $16: 55$ & $23: 17$ & 17 & 0.50 \\
\hline $01 / 25 / 04$ & $8: 12$ & $11: 06$ & 12 & 0.50 \\
\hline $01 / 26 / 04$ & $22: 15$ & $23: 48$ & 31 & 0.86 \\
\hline $01 / 26 / 04$ & 7:49 & $8: 58$ & 27 & 0.81 \\
\hline $01 / 26 / 04$ & $1: 52$ & $3: 51$ & 20 & 0.42 \\
\hline $01 / 27 / 04$ & $10: 50$ & $12: 36$ & 32 & 0.50 \\
\hline $01 / 27 / 04$ & $18: 11$ & $21: 18$ & 30 & 0.47 \\
\hline $01 / 27 / 04$ & $23: 25$ & $1: 30$ & 22 & 0.44 \\
\hline $01 / 27 / 04$ & $3: 35$ & $5: 36$ & 29 & 0.45 \\
\hline $01 / 28 / 04$ & 3:00 & $8: 05$ & 18 & 0.45 \\
\hline $01 / 29 / 04$ & $2: 07$ & $5: 13$ & 24 & 0.7 \\
\hline $01 / 29 / 04$ & $16: 56$ & $21: 22$ & 24 & 0.44 \\
\hline $01 / 29 / 04$ & $8: 27$ & $10: 23$ & 24 & 0.46 \\
\hline $01 / 30 / 04$ & $0: 32$ & $5: 18$ & 20 & 0.52 \\
\hline $01 / 30 / 04$ & $17: 23$ & $20: 11$ & 15 & 0.85 \\
\hline $02 / 07 / 04$ & $4: 50$ & $8: 31$ & 33 & 0.4 \\
\hline $02 / 07 / 04$ & $10: 27$ & $11: 30$ & 32 & 0.41 \\
\hline $02 / 12 / 04$ & $6: 19$ & $7: 50$ & 28 & 0.45 \\
\hline $02 / 21 / 04$ & 7:09 & 9:07 & 39 & 0.2 \\
\hline $02 / 21 / 04$ & $14: 58$ & $16: 58$ & 39 & 0.3 \\
\hline $02 / 21 / 04$ & $10: 33$ & $11: 35$ & 39 & 0.35 \\
\hline 03/08/04 & $1: 49$ & $4: 15$ & 37 & 0.35 \\
\hline $03 / 08 / 04$ & $7: 52$ & 9:52 & 34 & 0.37 \\
\hline 03/08/04 & $0: 16$ & $1: 30$ & 34 & 0.37 \\
\hline $03 / 08 / 04$ & $5: 19$ & $8: 08$ & 32 & 0.39 \\
\hline $03 / 12 / 04$ & $6: 35$ & $8: 07$ & 25 & 0.43 \\
\hline $03 / 22 / 04$ & $1: 32$ & $4: 49$ & 30 & 0.4 \\
\hline
\end{tabular}




\section{Appendix E}

\section{Historical Utility Costs for M-PRT}

The historical utility costs for M-PRT, shown in Table E.1 below, explain the natural gas price fluctuations.

Table E.1. Historical Utility Costs for M-PRT [25]

\begin{tabular}{|c|c|c|c|}
\hline Year & Natural Gas & Electricity & $\begin{array}{c}\text { Annual Utility Cost } \\
\text { (Gas and Electricity) }\end{array}$ \\
\hline 1997 & $\$ 136,722$ & $\$ 316,313$ & $\$ 453,035$ \\
\hline 1998 & $\$ 78,881$ & $\$ 272,686$ & $\$ 351,567$ \\
\hline 1999 & $\$ 93,867$ & $\$ 291,591$ & $\$ 385,458$ \\
\hline 2000 & $\$ 105,364$ & $\$ 269,130$ & $\$ 374,494$ \\
\hline 2001 & $\$ 125,324$ & $\$ 287,948$ & $\$ 413,272$ \\
\hline 2002 & $\$ 95,373$ & $\$ 261,559$ & $\$ 356,932$ \\
\hline 2003 & $\$ 329,382$ & $\$ 256,735$ & $\$ 586,117$ \\
\hline
\end{tabular}

Information provided by M-PRT Systems Engineering Manager. 